\title{
SPECTRAL STABILITY OF PERIODIC WAVE TRAINS OF THE KORTEWEG-DE VRIES/KURAMOTO-SIVASHINSKY EQUATION IN THE KORTEWEG-DE VRIES LIMIT
}

\author{
MATHEW A. JOHNSON, PASCAL NOBLE, L. MIGUEL RODRIGUES, \\ AND KEVIN ZUMBRUN
}

\begin{abstract}
We study the spectral stability of a family of periodic wave trains of the Korteweg-de Vries/Kuramoto-Sivashinsky equation $\partial_{t} v+v \partial_{x} v+\partial_{x}^{3} v+$ $\delta\left(\partial_{x}^{2} v+\partial_{x}^{4} v\right)=0, \delta>0$, in the Korteweg-de Vries limit $\delta \rightarrow 0$, a canonical limit describing small-amplitude weakly unstable thin film flow. More precisely, we carry out a rigorous singular perturbation analysis reducing the problem of spectral stability in this limit to the validation of a set of three conditions, each of which have been numerically analyzed in previous studies and shown to hold simultaneously on a non-empty set of parameter space. The main technical difficulty in our analysis, and one that has not been previously addressed by any authors, is that of obtaining a useful description for $0<\delta \ll 1$ of the spectrum of the associated linearized operators in a sufficiently small neighborhood of the origin in the spectral plane. This modulational stability analysis is particularly interesting, relying on direct calculations of a reduced periodic Evans function and using in an essential way an analogy with hyperbolic relaxation theory at the level of the associated Whitham modulation equations. A second technical difficulty is the exclusion of high-frequency instabilities lying between the $\mathcal{O}(1)$ regime treatable by classical perturbation methods and the $\gtrsim \delta^{-1}$ regime excluded by parabolic energy estimates.
\end{abstract}

\section{Contents}

1. Introduction

2. Expansion of periodic traveling-waves in the KdV limit

3. Stability with respect to high-frequency perturbations

4. Spectrum at the origin and modulation equations

Appendix A. High-frequency spectra: Th

Acknowledgements

References

Received by the editors February 28, 2012 and, in revised form, June 20, 2013.

2010 Mathematics Subject Classification. Primary 35B35, 35B10, 35 Q53.

The research of the first author was partially supported under NSF grant no. 1211183.

The research of the second author was partially supported by the French ANR Project no. ANR-09-JCJC-0103-01.

The stay of the third author in Bloomington was supported by French ANR project no. ANR09-JCJC-0103-01.

The research of the fourth author was partially supported under NSF grant no. DMS-0300487. 


\section{INTRODUCTION}

In this paper, we study the spectral stability of periodic wave trains of the Korteweg-de Vries/Kuramoto-Sivashinsky (KdV-KS) equation

$$
\partial_{t} u+u \partial_{x} u+\partial_{x}^{3} u+\delta\left(\partial_{x}^{2} u+\partial_{x}^{4} u\right)=0, \quad \forall t>0, \forall x \in \mathbb{R},
$$

with $0<\delta \ll 1$. When $\delta=0$, equation (1.1) reduces to the well-studied Kortewegde Vries (KdV) equation, which is an example of a completely integrable infinite dimensional Hamiltonian system. As such, the KdV equation is solvable by the inverse scattering transform, and serves as a canonical integrable equation in mathematical physics and applied mathematics describing weakly non-linear dynamics of long one dimensional waves propagating in a dispersive medium.

When $\delta>0$ on the other hand, equation (1.1) accounts for both dissipation and dispersion in the medium. In particular, for $0<\delta \ll 1$ it is known to model a thin layer of viscous fluid flowing down an incline, in which case it can be derived either from the shallow water equations

$$
\partial_{t} h+\partial_{x}(h u)=0, \quad \partial_{t}(h u)+\partial_{x}\left(h u^{2}+\frac{h^{2}}{2 F^{2}}\right)=h-u^{2}+\nu \partial_{x}\left(h \partial_{x} u\right)
$$

as $F \rightarrow 2^{+}$( $F$ being the Froude number, with $F=2$ the critical value above which steady constant-height flows are unstable) or from the full Navier-Stokes equations if $0<R-R_{c} \ll 1\left(R_{c}\right.$ being the critical Reynolds number above which steady Nusselt flows are unstable) in the small amplitude/large scale regime; see [Wi,YY] for more details. For other values of $\delta$, (1.1) serves as a canonical model for pattern formation that has been used to describe, variously, plasma instabilities, flame front propagation, or turbulence/transition to chaos in reaction-diffusion systems $[\mathrm{S} 1, \mathrm{~S} 2, \mathrm{SM}, \mathrm{K}, \mathrm{KT}$.

Here, our goal is to analyze the spectral stability of periodic traveling wave solutions of (1.1) with respect to small localized perturbations in the singular limit $\delta \rightarrow 0$. In this limit the governing equation (1.1) may be regarded as a dissipative (singular) perturbation of the $\mathrm{KdV}$ equation, for which it is known that all periodic traveling waves are spectrally stable to small localized perturbations; see $\mathrm{BD}, \mathrm{KSF}, \mathrm{Sp}$. However, as the limiting KdV equation is time-reversible (Hamiltonian), this stability is of "neutral" (neither growing nor decaying) type, and so it is not immediately clear whether the stability of these limiting waves carries over to stability of "nearby" waves in the flow induced by (1.1) for $|\delta| \ll 1$. Indeed, we shall see that, for different parameters, neutrally stable periodic KdV waves may perturb to either stable or unstable periodic KdV-KS waves, depending on the results of a rather delicate perturbation analysis.

Our analysis, mathematically speaking, falls in the context of perturbed integrable systems, a topic of independent interest. In this regard, it seems worthwhile to mention that the proof of stability of limiting $\mathrm{KdV}$ waves, and in particular the explicit determination of eigenvalues and eigenfunctions of associated linearized operators on which the present analysis is based, is itself a substantial problem that remained for a long time unsettled. Indeed, by an odd coincidence, both the original proof of spectral stability in $[\mathrm{KSF}, \mathrm{Sp}$ and a more recent proof of spectral and linearized stability in $[\mathrm{BD}$ ] (see also the restricted non-linear stability result [DK]) were accompanied by claims appearing at about the same time as instability of these waves, an example of history repeating itself and indirect indication of the difficulty of this problem. 
However, our motivations for studying this problem come very much from the physical applications to thin-film flow, and particularly the interesting metastability phenomena described in [PSU, BJRZ, BJNRZ3] (see Section 1.1 below). Interestingly, our resolution of the most difficult aspect of this problem, the analysis of the small-Floquet number/small- $\delta$ regime, is likewise motivated by the associated physics, in particular, by the formal Whitham equations expected to govern longwave perturbations of $\mathrm{KdV}$ waves, and an extended relaxation-type system formally governing the associated small- $\delta$ KdV-KS problem; see [NR2].

The identification of this structure, and the merging of integrable system techniques with asymptotic ODE techniques introduced recently in, e.g., JZ2, PZ, HLZ, BHZ (specifically, in our analysis of frequencies $|\lambda| \in\left[C, C \delta^{-1}\right]$ ), we regard as interesting contributions to the general theory that may be of use in related problems involving perturbed integrable systems. Our main contribution, though, is to the theory of thin-film flow, for which the singular limit $\delta \rightarrow 0$ appears to be the canonical problem directing asymptotic behavior.

We begin by defining the notion of spectral stability of periodic waves of the 4th order parabolic system KdV-KS, following BJNRZ1, as satisfaction of the following collection of non-degeneracy and spectral conditions:

- (H1) Let $\Omega \subset \mathbb{R}^{6}$ be an open set such that the map $H: \Omega \rightarrow \mathbb{R}^{3}$ taking $(X, b, c, q) \mapsto\left(u, u^{\prime}, u^{\prime \prime}\right)(X, b, c, q)-b$, where $\left(u, u^{\prime}, u^{\prime \prime}\right)(\cdot ; b, c, q)$ is the solution of

$$
\delta\left(u^{\prime \prime \prime}+u^{\prime}\right)+u^{\prime \prime}+\frac{1}{2} u^{2}-c u=q, \quad\left(u, u^{\prime}, u^{\prime \prime}\right)(0 ; b, c, q)=b,
$$

is well-defined. Let $(\bar{X}, \bar{b}, \bar{c}, \bar{q}) \in H^{-1}(\{0\})$; then $\bar{u}=u(. ; \bar{b}, \bar{c}, \bar{q})$ is a $\bar{X}$ periodic travelling wave of KdV-KS. We assume that $H$ is full rank at $(\bar{X}, \bar{b}, \bar{c}, \bar{q}) \in H^{-1}(\{0\})$. (Notice that this is precisely the condition of transversality of the periodic traveling wave as a solution of the traveling wave ODE.)

- (D1) $\sigma_{L^{2}(\mathbb{R})}(L) \subset\{\lambda \in \mathbb{C} \mid \Re(\lambda)<0\} \cup\{0\}$, where

$$
L=-\delta\left(\partial_{x}^{4}+\partial_{x}^{2}\right)-\partial_{x}^{3}-\partial_{x}(\bar{u}-\bar{c})
$$

denotes the linearized operator obtained by linearizing (1.1) about $\bar{u}=$ $u(. ; \bar{b}, \bar{c}, \bar{q})$.

- (D2) $\sigma_{L_{\text {per }}^{2}(0, \bar{X})}\left(L_{\xi}\right) \subset\left\{\left.\lambda \in \mathbb{C}|\Re \lambda \leq-\theta| \xi\right|^{2}\right\}$ for some $\theta>0$ and any $\xi \in[-\pi / \bar{X}, \pi / \bar{X})$, where

$$
L_{\xi}[f]=-\delta\left(\left(\partial_{x}+i \xi\right)^{4} f+\left(\partial_{x}+i \xi\right)^{2} f\right)-\left(\partial_{x}+i \xi\right)^{3} f-\left(\partial_{x}+i \xi\right)((\bar{u}-\bar{c}) f)
$$

denotes the associated Bloch operator with Bloch-frequency $\xi$.

- (D3) $\lambda=0$ is an eigenvalue of the Bloch operator $L_{0}$ of algebraic multiplicity two.

Under assumptions (H1) and (D3), standard spectral perturbation theory implies the existence of two eigenvalues $\lambda_{j}(\xi) \in \sigma\left(L_{\xi}\right)$ bifurcating from the $(\xi, \lambda)=(0,0)$ state of the form $\lambda_{j}(\xi)=i \beta_{j} \xi+o(\xi)$. Assumption (D1) ensures that $\beta_{j} \in \mathbb{R}$. Our final structural condition in our definition of spectral stability of periodic traveling wave solutions of (1.1) ensures the analyticity in $\xi$ of the critical curves $\lambda_{j}$ :

- (H2) The coefficients $\beta_{j}$ are distinct.

The above definition of spectral stability is justified by the results of [BJNRZ1, which state that, under the assumptions that (H1)-(H2) and (D1)-(D3) hold, the 
underlying wave $\bar{u}$ is $L^{1}(\mathbb{R}) \cap H^{4}(\mathbb{R}) \rightarrow L^{\infty}(\mathbb{R})$ non-linearly stable; moreover, if $\tilde{u}$ is any other solution of (1.1) with data sufficiently close to $\bar{u}$ in $L^{1}(\mathbb{R}) \cap H^{4}(\mathbb{R})$, for some appropriately prescribed $\psi$, the modulated solution $\tilde{u}(\cdot-\psi(\cdot, t), t)$ converges to $\bar{u}$ in $L^{p}(\mathbb{R}), p \in[2, \infty]$.

This is to be contrasted with the notion of spectral stability of periodic waves of Hamiltonian systems, which, up to genericity conditions analogous to (H1)-(H2) and (D3), amounts to the condition that the associated linearized operator analogous to $L$ has a purely imaginary spectrum. That is, in order that a (neutrally) stable periodic wave of $\mathrm{KdV}$ perturb under small $\delta>0$ to a stable periodic wave of $\mathrm{KdV}$ KS, its spectra must perturb from the imaginary axis into the stable (negative real part) complex half-plane.

The main goal of this paper, therefore, is to establish by rigorous singular perturbation theory a simple numerical condition guaranteeing the existence of periodic traveling wave solutions of (1.1) satisfying the above conditions (H1)-(H2) and (D1)-(D3) for sufficiently small $\delta>0$, more precisely, determining whether the neutrally stable periodic solutions of $\mathrm{KdV}$ perturb for small $\delta>0$ to stable or to unstable solutions of KdV-KS.

Remark 1.1. The methods used in BJNRZ1 to treat the dissipative case $\delta>0$, based on linearized decay estimates and variation of constants, are quite different from those typically used to show stability in the Hamiltonian case. The latter are typically based on Arnold's method, which consists of finding sufficiently many additional constants of motion, or "Casimirs", such that the relative Hamiltonian becomes positive or negative definite subject to these constraints (hence controlling the norm of perturbations); that is, additional constants of motion are used to effectively "excise" unstable (stable) eigenmodes of the second variation of the Hamiltonian. This approach is used in DK to show stability with respect to $n X$ periodic perturbations for arbitrary $n \in \mathbb{N}$, where $X$ denotes the period of the underlying $\mathrm{KdV}$ wave train. However, as $L^{2}$ spectra in the periodic case are purely essential, such unstable (stable) eigenmodes are uncountably many, and so it is unclear how to carry out this approach for general $L^{2}$ perturbations. Indeed, to our knowledge, the problem of stability of periodic $\mathrm{KdV}$ waves with respect to general $H^{s}$ perturbations remains open.

Remark 1.2. As we will see in Section 2 below, the set of all periodic traveling wave solutions of the KdV-KS equation (1.1) form a three parameter family parametrized by the period $X$ of the wave, the wave speed $c$, and the value of the wave profile at the origin. However, thanks to the translation and Galilean invariance of (1.1), it follows that the stability of a particular wave only depends on one parameter, namely, the period $X$ of the wave. Thus, when discussing the stability of periodic traveling wave solutions of the $\mathrm{KdV}-\mathrm{KS}$ equations we identify all waves of a particular period.

The spectral stability of periodic wave-train solutions of (1.1) itself has a long and interesting history of numerical and formal investigations. In [CDK], the authors studied numerically the spectral stability of periodic wave trains of

$$
\partial_{t} u+u \partial_{x} u+\gamma \partial_{x}^{3} u+\partial_{x}^{2} u+\partial_{x}^{4} u=0,
$$

which is, up to a rescaling, equation (1.1) with $\delta=\gamma^{-1}$, and they showed the stabilizing effect of strong dispersion (large $\gamma /$ small $\delta$ s). As $\gamma$ is increased from 0 
to $8(\delta \in(1 / 8, \infty))$, only one family of periodic waves of the Kuramoto-Sivashinsky equation survives and its domain of stability becomes larger and larger and seems to "converge" to a finite range $\left(X_{1}, X_{2}\right)$ with $X_{1} \approx 2 \pi / 0.74$ and $X_{2} \approx 2 \pi / 0.24$. In $[\mathrm{BN}]$, Bar and Nepomnyashchy studied formally the spectral stability of periodic wave trains of (1.1) as $\delta \rightarrow 0$, finding evidence for the existence of spectrally stable waves in the $\delta \rightarrow 0$ limit as well. More precisely, for a fixed Bloch wave number $\xi$ the non-zero eigenvalues $\left\{\lambda_{j}(\xi, \delta)\right\}_{j=1}^{\infty}$ of $L_{\xi}$ are formally expanded ${ }^{1}$ as

$$
\lambda_{j}(\delta, \xi)=\lambda_{j, 0}(\xi)+\delta \lambda_{j, 1}(\xi)+\mathcal{O}\left(\delta^{2}\right)
$$

for each $j \in \mathbb{N}$, where the $\left\{\lambda_{j, 0}(\xi)\right\}_{j=1}^{\infty} \subset \mathbb{R} i$ denote the eigenvalues associated with the stability of periodic waves of KdV, known explicitly (see [BD]), and the $\lambda_{j, 1}(\xi)$ are described in terms of elliptic integrals 2 Then, the authors verified numerically, using high-precision computations in MATHEMATICA (see [BN, Appendix B] and Appendix (A), that

$$
\sup _{\xi \in[-\pi / \bar{X}, \pi / \bar{X})} \sup _{j \in \mathbb{N}} \Re\left(\lambda_{j, 1}(\xi)\right)<0,
$$

consistent with stability, on the band of periods $\bar{X} \in\left(X_{1}, X_{2}\right)$ with $X_{1} \approx 8.49$ and $X_{2} \approx 26.17$, which are approximately the bounds found in CDK. Similar bounds were found numerically in [BJNRZ1] by completely different, direct Evans function, methods, with excellent agreement to those of [BN].

However, the study of Bar and Nepomnyashchy is only formal and, in particular, as mentioned in $[\overline{\mathrm{BN}}$, it is not valid in the neighbourhood of the origin $(\xi, \lambda)=$ $(0,0)$. Because of a lack of uniformity with respect to $\xi$ when $\xi$ goes to 0 , it seems at first glance that the expansion in $[\mathrm{BN}]$ is only uniformly valid for $|\xi|$ bounded away from zero. However, as we show in Section 4 after blowing up with respect to $\delta$ the $(\xi, \lambda)=(0,0)$ singularity, it turns out that the description (1.2) possesses an extension uniformly valid in a cone $0<\frac{\delta}{|\xi|} \ll 1$. Yet, even with this optimized justification, for a given $\delta>0$ it is still not possible to deduce directly from this expansion any spectral stability of an associated periodic wave train of (1.1) since the analysis misses a $\delta$-neighborhood of the origin in the space of the Floquet parameters $\xi$.

Likewise, the numerical study in Section 2 of BJNRZ1, which is not a singular perturbation analysis, but rather a high-precision computation down to small but positive $\delta 3$ gives information about $\delta \rightarrow 0$ only at finite scales, and hence in effect omits an $\mathcal{O}(\delta \times T O L)$ neighborhood of the origin, where $T O L$ is the relative precision of the computation. Thus, though very suggestive, neither of these computations gives conclusive results about stability in the $\delta \rightarrow 0$ limit, and, in particular, behavior on a $\delta$-neighborhood of the origin is not (either formally or numerically) described.

In this paper, we make rigorous the formal singular perturbation analysis that was done in $[\mathrm{BN}]$ and extend it to the frequency regimes that were omitted in $[\mathrm{BN}]$, completing the study of the spectrum at the origin and in the high-frequency regime. More precisely, we carry out a rigorous singular perturbation analysis reducing the problem to the study of Bloch parameters $\xi \in[C \delta, 2 \pi]$ (see Section

\footnotetext{
${ }^{1}$ As we shall see, such eigenvalues do expand in this way under condition (A1) below.

${ }^{2}$ See formula A.7 where $v_{0}$ is an (explicit) associated KdV eigenfunction and $w_{0}$ is explicitly given by A.4.

${ }^{3}$ Minimum value $\delta=.05$, as compared to $\delta=.125$ in [CDK]; see Table 3, BJNRZ1.
} 
3 for the definition of a Bloch parameter) and eigenvalues $|\Re \lambda| \leq C \delta,|\Im \lambda| \leq C$, $C>0$, sufficiently large, on which the computations of $[\mathrm{BN}$ may be justified by standard Fenichel-type theory.

The exclusion of high frequencies is accomplished by a standard parabolic energy estimate restricting $|\Im \lambda| \leq C \delta^{-3 / 4}$, followed by a second energy estimate on a reduced "slow", or "KdV", block restricting $|\Im \lambda| \leq C$; see Lemma 3.1 and Proposition 3.3 in Section 3.1 below. For related singular perturbation analyses using this technique of successive reduction and estimation, see for example [MZ, PZ, Z, JZ2 and especially [BHZ, Section 4. The treatment of small frequencies proceeds as usual by quite different techniques involving rather the isolation of "slow modes" connected with formal modulation and large-time asymptotic behavior.

At a technical level, this latter task appears quite daunting, being a twoparameter bifurcation problem emanating from a triple root $\lambda=0$ of the Evans function at $\xi=\delta=0$, where the Evans function $E(\lambda, \xi, \delta)$ (defined in (3.4) below) is an analytic function whose roots $\lambda$ for fixed $\delta>0$ and $\xi \in[-\pi / \bar{X}, \pi / \bar{X})$ comprise the $L^{2}$-spectrum of the linearized operator about the periodic solution. However, using the special structure of the problem, we are able to avoid the analysis of presumably complicated behavior on the main "transition regime" $\Lambda:=$ $\left\{(\delta, \xi)\left|C^{-1} \leq\right| \xi \mid / \delta \leq C\right\}, C \gg 1$, and only examine the two limits $|\xi| / \delta \rightarrow 0,+\infty$ on which the problem reduces to a pair of manageable one-parameter bifurcation problems of familiar types.

Specifically, we show that (small) roots $\lambda$ of the Evans function cannot cross the imaginary axis within $\Lambda$, so that stability properties need only be assessed on the closure of the $\Lambda$-complement, with the results then propagating by continuity from the boundary of $\Lambda$ to its interior. This has the further implication that stability properties on the wedges $|\xi| / \delta \ll 1$ and $|\xi| / \delta \gg 1$ are linked (through $\Lambda$ ), and so it suffices to check stability on the single wedge $|\xi| / \delta \gg 1$, where the analysis reduces to computations carried out in $[\mathrm{BN}]$. Indeed, the situation is simpler still: stability on the entire region $|\xi|, \delta \ll 1$ reduces by the above considerations to validity of a certain "subcharacteristic condition" relating characteristics of the Whitham modulation equations for $\mathrm{KdV}$ (the limit $|\xi| / \delta \rightarrow \infty$ ) and characteristics of a limiting reduced system as $\delta \rightarrow 0$ (the limit $|\xi| / \delta \rightarrow 0$ ).

As the above terminology suggests, there is a strong analogy in the regime $|\xi|, \delta \ll 1$ to the situation of symmetric hyperbolic relaxation systems and stability of constant solutions in the large time or small relaxation parameter limit [SK, Yo, Ze, for which a similar "non-crossing" principle reduces the question of stability to checking of Kawashima's genuine coupling condition, which in simple cases reduces to the subcharacteristic condition that characteristics of relaxation and relaxed system interlace. Indeed, at the level of Whitham modulation equations, the limit as $|\xi| / \delta \rightarrow \infty$ may be expressed as a relaxation from the Whitham modulation equations for $\mathrm{KdV}$ to the Whitham equations for fixed $\delta$ in the limit as $\delta \rightarrow 0$, a relation which illuminates both the role/meaning of the subcharacteristic condition and the relation between $\mathrm{KdV}$ and perturbed systems at the level of asymptotic behavior. These issues, which we regard as some of the most interesting and important observations of the paper, are discussed in Section 4.2.

The final outcome, and the main result of this paper, is that stability- whether spectral, linear, or non-linear- of periodic traveling wave solutions of (1.1) in the $K d V$ limit $\delta \rightarrow 0$ is completely determined by the formal high-frequency analysis and numerical results of $[\mathrm{BN}]$. 
To state our main result more precisely, we introduce three natural conditions that we assume hold simultaneously to obtain stability. Before stating these conditions, note, first, that by translational invariance of (1.1), two waves differing by a uniform translation share the same stability properties; thus, we will identify any two such waves throughout the analysis. Further, it is shown in EMR that there exists a two dimensional submanifold $\mathcal{M}$ of $\mathbb{R}^{3}$ such that if $(X, M, E) \in \mathcal{M}$, then the $X$-periodic traveling wave solution $u_{0}(\cdot ; X, M, E)$ of the $\mathrm{KdV}$ equation, unique up to translations, with $\int_{0}^{X} u_{0}(x) d x=M$ and $\int_{0}^{X} \frac{1}{2} u_{0}(x)^{2} d x=E$, continues, for $\delta$ sufficiently small, to a one-parameter family $\delta \mapsto u_{\delta}$, defined for $\delta$ sufficiently small, of $X$-periodic traveling wave solutions of the KdV-KS equation (1.1) such that $\int_{0}^{X} u_{\delta}(x) d x=M$, with $u_{\delta} \rightarrow u_{0}$ uniformly on $[0, X]$ as $\delta \rightarrow 0$; see Proposition 2.4 below for details. In particular, for each period $X$ there exists (up to translations) a one-parameter family of $X$-periodic traveling wave solutions of the $\mathrm{KdV}$ equation that can be continued to $X$-periodic solutions $u_{\delta}$ of KdV-KS. However, we notice that by the Galilean invariance of (1.1) the stability of a given wave $u_{\delta}$ depends only on the period $X$ and not on the quantities $M$ and $E$ associated with the limiting $\mathrm{KdV}$ wave train. This motivates us to define the set

$$
\mathcal{W}:=\left\{X>0 \mid(X, M, E) \in \mathcal{M} \text { for some }(M, E) \in \mathbb{R}^{2}\right\} .
$$

Our first condition on admissible periods $X \in \mathcal{W}$, related to the non-degneracy of the Bloch spectrum for the associated KdV linearized operator, is as follows:

- A period $X \in \mathcal{W}$ is said to satisfy condition (A1) if there exists an $\left(M_{1}, E_{1}\right)$ $\in \mathbb{R}^{2}$ with $\left(X, M_{1}, E_{1}\right) \in \mathcal{M}$ such that all the non-zero eigenvalues of the linearized (Bloch) KdV operators

$$
L_{\mathrm{KdV}, \xi}\left[u_{0}\right]:=\left(\partial_{x}+i \xi\right)\left(-\left(\partial_{x}+i \xi\right)^{2}-u_{0}+c\right): L_{\mathrm{per}}^{2}(0, X) \rightarrow L_{\mathrm{per}}^{2}(0, X)
$$

about the associated limiting $\mathrm{KdV}$ wave $u_{0}=u_{0}\left(\cdot ; X, M_{1}, E_{1}\right)$, considered with compactly embedded domain $H_{\text {per }}^{3}(0, X)$, are simple for each $\xi \in[-\pi / X, \pi / X)$ and $\lambda=0$ is an eigenvalue of $L_{\mathrm{KdV}, \xi}\left[u_{0}\right]$ only if $\xi=0$, in which case it is an eigenvalue of algebraic multiplicity three 4

Notice by the above comments that if the condition in (A1) holds for some $(M, E) \in$ $\mathbb{R}^{2}$ with $(X, M, E) \in \mathcal{M}$, then it necessarily holds for all $(M, E) \in \mathbb{R}^{2}$ with $(X, M, E) \in \mathcal{M}$.

Condition (A1) was shown to hold for a particular period in BD, where the authors derive explicit formulas for the eigenvalues of $L_{\mathrm{KdV}, \xi}$; see Figure 2 and the surrounding discussion in $[\mathrm{BD}$. Furthermore, this condition is verified 5 for a particular wave by the plot in Figure 1, where we plot the Bloch spectrum as a function of the Bloch-frequency $\xi$ for a $\mathrm{KdV}$ cnoidal wave with period $X \approx 9.39$. The first main objective in our analysis is to rigorously justify, given a family of periodic traveling wave solutions $u_{\delta}$ with period satisfying condition (A1), that the non-zero Bloch eigenvalues of the linearized KdV-KS operator can be expanded

\footnotetext{
${ }^{4}$ It was shown in Section 5 of $[\mathrm{BrJK}]$ that the origin is always an eigenvalue of $L_{\mathrm{KdV}, 0}\left[u_{0}\right]$ with algebraic multiplicity three.

${ }^{5}$ In Figure 1 the spectrum is recovered by projecting the graph onto the vertical axis. Obviously we do not plot the full unbounded spectrum, but notice that the non-displayed monotone branches singly cover the remainder of the imaginary axis, hence the only possibility for eigenvalues of the associated KdV Bloch operators to not be simple is in a neighborhood of the origin.

${ }^{6}$ More precisely, for $k=0.9$ in Proposition 2.4
} 


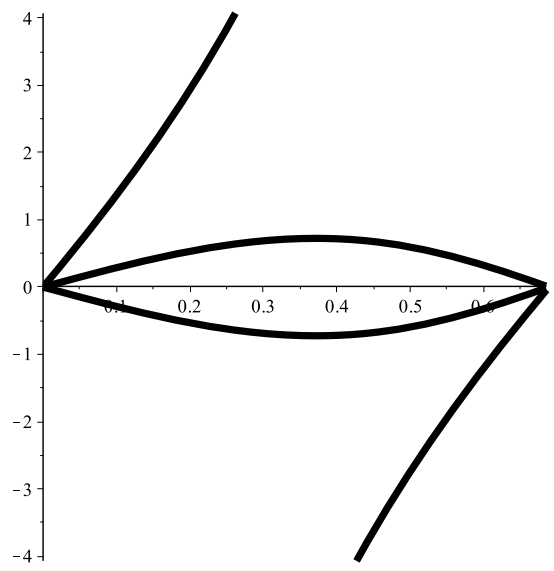

FiguRE 1. Here, we plot the imaginary part of the (purely imaginary) eigenvalues (vertical axis) of the $\mathrm{KdV}$ linearized (Bloch) operator $\mathcal{L}_{\xi}[\bar{u}]$ about an $X$-periodic traveling wave solution of the $\mathrm{KdV}$ equation that continues as a solution of (1.1) for $0<\delta \ll 1$ as a function of $\xi \in[0,2 \pi / X)$ (horizontal axis), where here we take $k=0.9$, i.e. $X \approx 9.39$, in Proposition 2.4. The spectrum of $\mathcal{L}[\bar{u}]$ on $L^{2}(\mathbb{R})$ is recovered by projecting this graph onto the vertical axis. From this figure, it is clear that for each $\xi \in(0,2 \pi / X)$ the eigenvalues of $\mathcal{L}_{\xi}$ are simple, verifying condition (A1) in this particular case.

analytically in $\delta$ as $\delta \rightarrow 0$ for each fixed $\xi \in[-\pi / X, \pi / X)$; this is the content of Corollary 3.8 below 7

Our next condition concerns the nature of the eigenvalues near the origin of the linearized (Bloch) KdV operators $L_{\mathrm{KdV}, \xi}\left[u_{0}\right]$ about an $X$-periodic cnoidal wave $u_{0}$ of the $\mathrm{KdV}$ equation when $X \in \mathcal{W}$ and $|\xi| \ll 1$. Assuming that condition (A1) holds for $X$, the origin is an eigenvalue of the operator $L_{\mathrm{KdV}, 0}\left[u_{0}\right]$ with algebraic multiplicity three. Furthermore, this triple eigenvalue is known to break differentiably in $\xi$ for $|\xi| \ll 1$. More precisely, there are three critical modes $\left\{\lambda_{\mathrm{KdV}, j}(\xi)\right\}_{j=1,2,3}$ of the KdV linearized operator that can be expanded for $|\xi| \ll 1$ as

$$
\lambda_{\mathrm{KdV}, j}(\xi)=i \alpha_{j}(\xi) \xi=i \alpha_{j}^{0} \xi+o(|\xi|)
$$

for some real numbers $\alpha_{j}^{0} \in \mathbb{R}$; see, for instance, $\mathrm{BrJ}$ ] for details. The second condition in our analysis is as follows:

- A period $X \in \mathcal{W}$ is said to satisfy condition (A2) if it satisfies condition (A1) and if there exists $\left(M_{2}, E_{2}\right) \in \mathbb{R}^{2}$ with $\left(X, M_{2}, E_{2}\right) \in \mathcal{M}$ such that the real numbers $\left\{\alpha_{j}^{0}\right\}_{j=1,2,3}$ in (1.3) associated with the linearized operator $L_{\mathrm{KdV}, \xi}\left[u_{0}\left(\cdot ; X, M_{2}, E_{2}\right)\right]$ are distinct.

${ }^{7}$ This fact seems to have been taken for granted in the formal analysis of [BN]. As we will see, however, our proof relies on condition (A1) holding for the particular wave we are studying, thus we must either assume that the non-degeneracy condition (A1) holds for this wave or else prove such a condition to make this expansion rigorous. 
As above, if (A2) holds for some $(M, E) \in \mathbb{R}^{2}$ with $(X, M, E) \in \mathcal{M}$, then it necessarily holds for all $(M, E) \in \mathbb{R}^{2}$ with $(X, M, E) \in \mathcal{M}$.

In $\mathrm{BrJK}$, it was numerically verified that all periods of all periodic traveling wave solutions of the KdV equation satisfy condition (A2) 8 Furthermore, in Section 4.3 below we provide an alternate numerical verification of condition (A2) for all periods of the $\mathrm{KdV}$ cnoidal waves by using the well-known identification of the $\alpha_{j}^{0}$ in (1.3) to the characteristic speeds of the associated KdV Whitham modulation system; in particular, see Figure 3 in Section 4.3. The validity of condition (A2) will prove to be crucial in our analysis of the spectrum of the KdV-KS linearized operator $L_{\xi}$ near the origin for $|\xi| \ll 1$; see Section 4 below.

Our final condition is the stability criterion derived formally by Bar and Nepomnyashchy $\mathrm{BN}$. More specifically, if $X \in \mathcal{W}$ satisfies condition (A1) we will show that for each family of $X$-periodic traveling wave solutions $u_{\delta}$ of (1.1) the nonzero eigenvalues of the Bloch operators $L_{\xi}\left[u_{\delta}\right]$ admit an analytic expansion in $\delta$ for $0<\delta \ll 1$ of the form (1.2) for each fixed $\xi \in[-\pi / X, \pi / X)$. Indeed, if $X \in \mathcal{W}$, then to each pair $\left(\xi, \lambda_{0}\right)$ with $\lambda_{0} \in \sigma\left(L_{\mathrm{KdV}, \xi}\left[u_{\delta}\right]\right) \backslash\{0\}$ and $\xi \in[-\pi / X, \pi / X)$ there is a unique spectral curve $\lambda\left(\xi, \lambda_{0}, \delta\right)$ bifurcating from $\lambda_{0}$ analytically in $\delta$ of the form

$$
\lambda\left(\xi, \lambda_{0}, \delta\right)=\lambda_{0}+\delta \lambda_{1}\left(\xi, \lambda_{0}\right)+\mathcal{O}\left(\delta^{2}\right)
$$

Notice that this parametrization of the spectrum of the linearized KdV-KS operator by the pair $\left(\xi, \lambda_{0}\right)$ is well defined under the assumption that $X$ satisfies condition (A1). The final condition, stating that a particular wave satisfies the stability criterion that was numerically investigated in $[\mathrm{BN}]$, is as follows. This is stated precisely as follows:

- A period $X \in \mathcal{W}$ is said to satisfy condition (A3) if it satisfies condition (A1) and if there exists $\left(M_{3}, E_{3}\right) \in \mathbb{R}^{2}$ with $\left(X, M_{3}, E_{3}\right) \in \mathcal{M}$ such that the function

$$
\operatorname{Ind}(X):=\sup _{\substack{\lambda_{0} \in \sigma\left(L_{\mathrm{KdV}, \xi}\left[u_{0}\left(\cdot ; X, M_{3}, E_{3}\right)\right]\right) \backslash\{0\} \\ \xi \in[-\pi / X, \pi / X)}} \Re\left(\lambda_{1}\left(\xi, \lambda_{0}\right)\right)
$$

satisfies $\operatorname{Ind}(X)<0$.

As above, if (A3) holds for some $(M, E) \in \mathbb{R}^{2}$ with $(X, M, E) \in \mathcal{M}$, then it necessarily holds for all $(M, E) \in \mathbb{R}^{2}$ with $(X, M, E) \in \mathcal{M}$.

Remark 1.3. In Appendix $\mathrm{A}$, we review the numerical calculations in $\mathrm{BN}$ concerning condition (A3). In particular, we recall there the explicit formula for $\lambda_{1}\left(\xi, \lambda_{0}\right)$ used by Bar and Nepomnyashchy [BN].

Remark 1.4. We note in passing that, in principle, given a period $X>0$ each of the conditions (A1)-(A3) can be verified for a different $(M, E) \in \mathbb{R}^{2}$ such that $(X, M, E) \in \mathcal{M}$. In practice, however, it seems easier to verify them simultaneously for a fixed wave, i.e. for a fixed $(M, E) \in \mathbb{R}^{2}$ with $(X, M, E) \in \mathcal{M}$. That is, one would find a wave satisfying (A1) and then verify that it also satisfies (A2) and (A3).

\footnotetext{
${ }^{8} \mathrm{In}\left[\mathrm{BrJK}\right.$, the $\alpha_{j}^{0}$ are distinct so long as the "modulational instability index" $\Delta_{\mathrm{MI}}$ is non-zero, which was (numerically) shown to hold for all cnoidal wave solutions of KdV in Section 5.1 of BrJK.
} 
As we will see, the validity of conditions (A1) and (A3) provides us with a "highfrequency" stability result, allowing us to verify spectral stability of a given wave outside a sufficiently small neighborhood of the origin in the spectral plane; see Section 3.3 and, in particular, Corollary 3.10. Furthermore, we will also see that conditions (A2) and (A3) imply a set of "subcharacteristic" conditions that allows us to obtain a low-frequency stability result, verifying spectral stability in a sufficiently small neighborhood of the origin; see Section 4.1. Together then, recalling Remark 1.2 these complementary results provide a rigorous proof of spectral stability of a given family of the $X$-periodic "near-KdV" traveling wave solution of (1.1), in the case that the period $X \in \mathcal{W}$ satisfies conditions (A1)-(A3) simultaneously. Notice, however, that it is a highly non-trivial task to check that there exists a period $X \in \mathcal{W}$ that satisfies (A1), (A2), and (A3); indeed, to the best of our knowledge no such proof exists. Nevertheless, there are many well-founded numerical results suggesting that there is a non-empty open set of periods for which (A1)-(A3) hold; see, for instance, BN, BJNRZ1, CDK, together with the numerical experiments in Section 4.3 of this paper. Still, we consider the rigorous verification of these assumptions for a non-empty common set of periods to be an important open problem.

With the above motivation, we define $\mathcal{P}$ to be the set of all periods $X \in \mathcal{W}$ that simultaneously satisfy conditions (A1)-(A3). Note that $\mathcal{P}$ is open. Furthermore, it is natural to expect, based on the aforementioned numerical evidence, that the set $\mathcal{P}$ is non-empty. Indeed, by the above discussion, it is expected that $\mathcal{P}$ is a connected interval $\left(X_{1}, X_{2}\right)$ with $X_{1} \approx 8.49$ and $X_{2} \approx 26.17$. Now, we can state precisely the main result of our analysis.

Theorem 1.5. For each $X \in \mathcal{P}$, there exists a real number $0<\delta_{0}(X) \ll 1$ such that for each $0<\delta<\delta_{0}(X)$, the non-degeneracy and spectral stability conditions (H1)-(H2) and (D1)-(D3) hold for all X-periodic traveling wave solutions $u_{\delta}$ of (1.1), as constructed in EMR (see Proposition 2.4 below). Moreover, $\delta_{0}(\cdot)$ can be taken to be uniform on compact subsets of $\mathcal{P}$.

Remark 1.6. Though the choice of $\delta_{0}$ can be taken to be uniform on compact subsets of $\mathcal{P}$, as mentioned above, we expect that $\mathcal{P}=\left(X_{1}, X_{2}\right)$ with $X_{1} \approx 8.49$ and $X_{2} \approx 26.17$, in which case one would have $\delta_{0}(X) \rightarrow 0$ as $X \searrow X_{1}$ or $X \nearrow X_{2}$.

Remark 1.7. Our proof yields another form of uniformity. If $\mathcal{K}$ is a compact subset of the subset of $\mathcal{W}$ on which (A1)-(A2) hold, then there exists $C>0$ such that, for any $X \in \mathcal{K}$, condition (A3) is equivalent to

$$
\sup _{\substack{\lambda_{0} \in \sigma\left(L_{\mathrm{KdV}, \xi}\left[u_{0}\right]\right) \backslash\{0\} \\ \xi \in[-\pi / X, \pi / X) \\|\lambda| \leq C}} \Re\left(\lambda_{1}\left(\xi, \lambda_{0}\right)\right)<0 .
$$

Since, as we shall prove, $\lambda_{1}\left(\xi, \lambda_{0}\right)$ converges, as $\left(\xi, \lambda_{0}\right) \rightarrow(0,0)$, to one of three possible limits, depending on the spectral curve followed by $\left(\xi, \lambda_{0}\right)$, the validation of (A3) is indeed uniformly reduced to the sign evaluation of an explicit function on a compact set.

1.1. Discussion and open problems. To yield stability, our Theorem 1.5 should be completed with an investigation of conditions (A1)-(A3), and especially of condition (A3). Thus, given the present state of the art, it should be combined with the numerical investigations of $[\mathrm{BN}]$. Together this does not constitute a numerical 
proof, but rather a "numerical demonstration", in the sense that the computations of $[\mathrm{BN}]$ on which we ultimately rely for evaluation of the sign of $\operatorname{Ind}(X)$ are carried out with high precision and great numerical care, but not with interval arithmetic in a manner yielding guaranteed accuracy. However, there is no reason that such an analysis could not be carried out — we point for example to the computations of $[\mathrm{M}]$ in the related context of stability of radial KdV-KS waves - and, given the fundamental nature of the problem, this seems an important open problem for further investigation.

Indeed, numerical proof of stability or instability for arbitrary non-zero values of $\delta$, verifying the numerical conclusions of [BJNRZ1, or of Evans computations in general, though considerably more involved, seems also feasible, and another important direction for future investigations.

The particular limit $\delta \rightarrow 0$ studied here has special importance, we find, as a canonical limit that serves (as discussed at the beginning of the introduction) as an organizing center for other situations/types of models as well, and it has indeed been much studied; see, for example, [EMR, $, \mathrm{BN}, \mathrm{PSU}$, and the references therein. As discussed in PSU, BJNRZ3, BJRZ, it is also prototypical of the interesting and somewhat surprising behavior of inclined thin film flows that solutions often organize time-asymptotically into arrays of "near-solitary wave" pulses, despite the fact that individual solitary waves, since their endstates necessarily induce unstable essential spectrum 9 are clearly unstable.

To pursue the analogy between modulational behavior and solutions of hyperbolic-parabolic conservation or balance laws that has emerged in $\mathrm{OZ}$, Se, BJNRZ1,BJNRZ2, etc., and, indeed, through the earlier studies of [FST] or the still earlier work of Whitham [W], we feel that the KdV limit $\delta \rightarrow 0$ of (1.1) plays a role for small-amplitude periodic inclined thin film flow analogous to that played by Burgers equation for small-amplitude shock waves of general systems of hyperbolicparabolic conservation laws, and the current analysis a role analogous to that of Goodman's analysis in Go1, Go2 of spectral stability of general small-amplitude shock waves by singular perturbation of Burgers shocks 10

The difference from the shock wave case is that, whereas, up to Galilean and scaling invariances, the Burgers shock profile is unique, there exists up to invariances a one-parameter family of periodic waves of $\mathrm{KdV}$, indexed by the period $X$, of which only a certain range are stable. Moreover, whereas the Burgers shock profile is described by a simple tanh function, periodic $\mathrm{KdV}$ waves are described by a more involved parametrization involving elliptic functions. Thus, the study of periodic waves is inherently more complicated, simply by virtue of the number of cases that must be considered and by the complexity of the waves involved. Indeed, in contrast to the essentially geometric proof of Goodman for shock waves, we here find it necessary to use in essential ways certain exact computations coming from the integrability/inverse scattering formalism of the underlying KdV equation.

Plan of the paper. In Section 2, we recall how to compute an expansion of periodic waves of KdV-KS in the limit $\delta \rightarrow 0$ by using Fenichel singular perturbation theory [EMR. In Section [3, we analyze the stability of the spectrum of a

\footnotetext{
${ }^{9} \mathrm{~A}$ straightforward Fourier transform computation reveals that all constant solutions are unstable.

${ }^{10}$ See also the related $[\mathrm{PZ}$ FreS], more in the spirit of the present analysis.
} 
given $X$-periodic traveling wave solution $u_{\delta}$, as constructed in Section 3 for all $\delta$ sufficiently small, outside of a sufficiently small neighborhood of the origin in the spectral plane; at times, we will refer to this as a "high-frequency" stability result. This is accomplished by first deriving a priori estimates on the size of the unstable spectrum of $L\left[u_{\delta}\right]$ showing, in particular, that such unstable spectra are necessarily of order $\mathcal{O}(1)$ as $\delta \rightarrow 0$; see Proposition 3.3 and Corollary 3.5. Then, in Proposition 3.7 and Corollary 3.8, we compute an expansion of both the Evans function and high-frequency spectra with respect to $\delta$ as $\delta \rightarrow 0$. This analysis holds true except in a neighborhood of the origin from which spectral curves bifurcate. In Section [4 we compliment the high-frequency analysis of Section 3 by computing asymptotic expansions of the spectral curves in the neighborhood of the origin and show that spectral stability is related to subcharacteristic conditions for a Whitham's modulation system of relaxation type, as conjectured in [NR2].

Note added in proof. Since the acceptance of this paper, Barker [B] has established by rigorous numerical proof that for $X \in[6.285,48.36]$, there is a single stability range (i.e. periods for which conditions (A1)-(A3) hold) given by $\left[X_{l}, X_{r}\right]$ where $X_{l} \in[8.43,8.45]$ and $X_{r} \in[26.0573,26.0575]$, largely completing the stability analysis of small amplitude waves and answering the open problem posed in the discussion section above. It remains to treat the $X \rightarrow 2 \pi$ and $X \rightarrow \infty$ limits, which should be accessible by more standard (non-numerical) techniques.

\section{ExPANSION OF PERIODIC TRAVELING-WAVES IN THE KDV LIMIT}

For $0<\delta \ll 1$, equation (1.1) is a singular perturbation of the Korteweg-de Vries equation

$$
\partial_{t} u+u \partial_{x} u+\partial_{x}^{3} u=0
$$

where the periodic traveling wave solutions may be described with the help of the Jacobi elliptic functions. In EMR, periodic traveling wave solutions of (1.1) are found, $\delta$-close to periodic traveling wave solutions of (2.1), and, furthermore, an expansion of these solutions with respect to $\delta$ is obtained. We begin our analysis by briefly recalling the details of this expansion. Notice that (1.1) admits traveling wave solutions of the form $u(x, t)=U(x-c t)$, provided the profile $U$ satisfies the equation

$$
(U-c) U^{\prime}+U^{\prime \prime \prime}+\delta\left(U^{\prime \prime}+U^{\prime \prime \prime \prime}\right)=0,
$$

where here' denotes differentiation with respect to the traveling variable $\theta=x-c t$. Due to the conservative nature of (1.1) this profile equation may be integrated once yielding

$$
\frac{U^{2}}{2}-c U+U^{\prime \prime}+\delta\left(U^{\prime}+U^{\prime \prime \prime}\right)=q,
$$

where $q \in \mathbb{R}$ is a constant of integration. By introducing $x=U, y=U^{\prime}$ and $z=U^{\prime \prime}+U$, we may write (2.2) as the equivalent first order system

$$
x^{\prime}=y, \quad y^{\prime}=z-x, \quad \delta z^{\prime}=-z+q+(c+1) x-\frac{x^{2}}{2} .
$$

Setting $\delta=0$ in (2.3) yields the slow system

$$
z=q+(c+1) x-\frac{x^{2}}{2}, \quad x^{\prime}=y, \quad y^{\prime}=q+c x-\frac{x^{2}}{2},
$$


which is equivalent to the planar, integrable system governing the traveling wave profiles for the KdV equation (2.1). By using the well-known Fenichel theorems, one is able to justify the reduction and continue the resulting $\mathrm{KdV}$ profiles for $0<\delta \ll 1$. To this end, we define

$$
M_{0}=\left\{(x, y, z) \in \mathbb{R}^{3} \mid z=q+(c+1) x-\frac{x^{2}}{2}=: f_{0}(x)\right\},
$$

and recognize this as the slow manifold associated with (2.3). It is readily checked that this manifold is normally hyperbolic attractive, and so a standard application of the Fenichel theorems yields the following proposition.

Proposition 2.1. Let $O \subset \mathbb{R}^{2}$ be a bounded open set and $r \geq 1$ a (arbitrary) positive integer. There exists $\delta_{0}>0$ such that for all $\delta \in\left(0, \delta_{0}\right)$, there is a slow manifold $M_{\delta}$ invariant under the flow of (2.3) that is written as $M_{\delta}=$ $\left\{(x, y, z) \in \mathbb{R}^{3} \mid z=f_{\delta}(x, y)\right\}$, where the function $f_{\delta}$ is $C^{r}$ both in $(x, y) \in O$ and $\delta \in\left[0, \delta_{0}\right)$ variables. Moreover, $f_{\delta}$ expands as

$$
\begin{aligned}
& f_{\delta}(x, y)=f_{0}(x)+\delta f_{1}(x, y)+\delta^{2} f_{2}(x, y, \delta) \\
& f_{1}(x, y)=x y-(c+1) y, \quad f_{2}(x, y, 0)=-y^{2}+(c+1-x)\left(q+c x-\frac{x^{2}}{2}\right) .
\end{aligned}
$$

Remark 2.2. Notice that the specific choice of $r \geq 1$ of regularity in the above proposition is arbitrary, but that the size of $\delta_{0}$ and the particular choice of $f_{\delta}$ are expected, of course, to depend on the specific choice of $r$. In particular, as $r \rightarrow \infty$ we expect $\delta_{0} \rightarrow 0$. This flexibility being able to prescribe an arbitrarily large degree of regularity on $f$ will be important in our analysis. In particular, at any point we can make $r$ larger (but finite) by simply possibly choosing $\delta_{0}$ smaller (but still non-zero).

The expansion of $f_{\delta}$ is obtained by inserting $z=f_{\delta}(x, y)$ into (2.3) and identifying the powers in $\delta$. Then by plugging this expansion into $(2.3)_{2}$, one finds the reduced planar system

$$
x^{\prime}=y, \quad y^{\prime}=q+c x-\frac{x^{2}}{2}+\delta(x y-(c+1) y)+\delta^{2} f_{2}(x, y, \delta)
$$

or equivalently the scalar equation

$$
x^{\prime \prime}=q+c x-\frac{x^{2}}{2}+\delta(x-c-1) x^{\prime}+\delta^{2} f_{2}\left(x, x^{\prime}, \delta\right) .
$$

Equation (2.6) is a regularly perturbed problem. Then, fixing $r \geq 1$ as an arbitrary positive integer, it is easily proved that the $x$ solution of $(2.6)$ is $C^{r}$ in the variables $\theta=x-c t \in \mathbb{R}$ and $\delta \in\left[0, \delta_{0}\right)$ for some $\delta_{0}$ sufficiently small. Now, we seek an asymptotic expansion of the solutions of (2.5) in the limit $\delta \rightarrow 0$. An easy way of doing these computations to any order with respect to $\delta$ is to follow the formal computations in $[\mathrm{BN}$, which are now justified here with Fenichel's theorems. To begin, notice that when $\delta=0$ the periodic solutions $x=x_{0}$ with wave speed $c=c_{0}$ of (2.6) agree with those of the KdV equation (2.1), which are given explicitly by

$$
x_{0}\left(\theta ; \phi, \kappa, k, a_{0}\right)=a_{0}+12 k^{2} \kappa^{2} \mathrm{cn}^{2}(\kappa(\theta+\phi), k), \quad c_{0}=a_{0}+8 \kappa^{2} k^{2}-4 \kappa^{2},
$$

where $\operatorname{cn}(\cdot, k)$ is the Jacobi elliptic cosine function with elliptic modulus $k \in[0,1)$ and $\kappa>0, a_{0}, \phi$ are arbitrary real constants related to the Lie point symmetries of (2.1); see [BD. Thus, the set of periodic traveling wave solutions of (2.1) forms a four dimensional manifold (3 dimensional up to translations) parameterized by 
$a_{0}, \kappa, k$, and $\phi$. Note that such solutions are $2 K(k) / \kappa$-periodic, where $K(k)$ is the complete elliptic integral of the first kind.

Remark 2.3. The parameterization of the periodic traveling wave solutions of the $\mathrm{KdV}$ equation given in (2.7) is consistent with the calculations in $\mathrm{BD}$, where the authors verify the spectral stability of such solutions to localized perturbations using the complete integrability of the governing equation. However, this parameterization is not the same as that given in $[\mathrm{BN}]$, whose numerical results our analysis ultimately relies on. Indeed, in [BN] the periodic traveling wave solutions of (2.1) are given (up to rescaling 11 ) as

$$
X_{\mathrm{BN}}\left(\theta ; \theta_{0}, q, k\right)=\frac{q^{2} K(k)^{2}}{3 \pi^{2}}\left(\operatorname{dn}^{2}\left(\frac{\left(\theta-\theta_{0}\right) q K(k)}{\pi}, k\right)-\frac{E(k)}{K(k)}\right),
$$

where $\operatorname{dn}(\cdot, k)$ denotes the Jacobi dnoidal function with elliptic modulus $k \in[0,1)$, and $K(k)$ and $E(k)$ denote the complete elliptic integrals of the first and second kind, respectively. Nevertheless, using the identity

$$
k^{2} \operatorname{cn}^{2}(x, k)=\operatorname{dn}^{2}(x, k)-\left(1-k^{2}\right)
$$

we can rewrite (2.7) as

$$
x_{0}(\theta)=12 \kappa^{2}\left(\operatorname{dn}^{2}(\kappa(\theta+\phi), k)+\frac{u_{0}}{12 \kappa^{2}}-\left(1-k^{2}\right)\right),
$$

which, upon setting $\kappa=\frac{q K(k)}{\pi}, \phi=-\theta_{0}$, and choosing $u_{0}$ so that

$$
\frac{u_{0}}{12 \kappa^{2}}-\left(1-k^{2}\right)=-\frac{E(k)}{K(k)},
$$

we see that $x_{0}(\theta)=X_{\mathrm{BN}}(\theta)$. Thus, there is no loss of generality in choosing one parameterization over the other. Furthermore, the numerical results of [BN] carry over directly to the cnoidal wave parameterization chosen here.

Next, we consider the case $0<\delta \ll 1$. To begin we seek conditions guaranteeing that periodic traveling wave solutions of (1.1) exist for sufficiently small $\delta$. Multiplying both sides by $x^{\prime}$ and rearranging, we find that equation (2.6) may be written as

$$
\frac{d}{d \theta}\left(\frac{\left(x^{\prime}\right)^{2}}{2}+\frac{x^{3}}{6}-c \frac{x^{2}}{2}-q x\right)=\delta\left(x\left(x^{\prime}\right)^{2}-(c+1)\left(x^{\prime}\right)^{2}\right)+\mathcal{O}\left(\delta^{2}\right)
$$

hence a necessary condition for the existence of an $L$-periodic solution to (2.6) is

$$
\int_{0}^{L}\left(x_{0}(\theta)\left(x_{0}^{\prime}(\theta)\right)^{2}-(c+1)\left(x_{0}^{\prime}(\theta)\right)^{2}\right) d \theta=0 .
$$

By a straightforward computation using integration by parts and (2.6), (2.9) can be simplified to the selection principle

$$
\int_{0}^{L}\left(x_{0}^{\prime \prime}(\theta)\right)^{2} d \theta=\int_{0}^{L}\left(x_{0}^{\prime}(\theta)\right)^{2} d \theta
$$

\footnotetext{
${ }^{11} \mathrm{In}\left[\mathrm{BN}\right.$, the authors consider the $\mathrm{KdV}$ equation in the form $\tilde{u}_{t}+6 \tilde{u} \tilde{u}_{x}+\tilde{u}_{x x x}=0$, which is equivalent to 2.1] via the simple rescaling $\tilde{u} \mapsto \frac{1}{6} u$.
} 
or, equivalently,

$$
\kappa^{2}=\frac{\int_{0}^{2 K(k)}\left[\left(c n^{2}\right)^{\prime}\right]^{2}(y) d y}{\int_{0}^{2 K(k)}\left[\left(c n^{2}\right)^{\prime \prime}\right]^{2}(y) d y}=: F^{2}(k) .
$$

Using the implicit function theorem, one can show that if (2.11) is satisfied, there exists a periodic solution $x^{\delta}$ of (2.6) which is $\delta$ close to $x_{0}$. As a result, we obtain a 3 dimensional set of periodic solutions to (1.1) parametrized by $u_{0}, \phi$ and either $k$ or $\kappa$. Note that the limit $\kappa \rightarrow 0$ (i.e. $k \rightarrow 1$ ) corresponds to a solitary wave and $\kappa \rightarrow 1$ (i.e. $k \rightarrow 0$ ) corresponds to small amplitude solutions (or equivalently to the onset of the Hopf bifurcation branch).

The above observations lead us to the following proposition.

Proposition 2.4 ([EMR $]$ ). Given any positive integer $r \geq 1$, there exists a $\delta_{0}>0$ such that the periodic traveling wave solutions $u_{\delta}(\theta), \theta=x-c t$, of (1.1) are analytic functions of $\theta \in \mathbb{R}$ and $C^{r}$ functions of $\delta \in\left[0, \delta_{0}\right)$. Furthermore, taking $r \geq 3$, we find that as $\delta \rightarrow 0$ the profiles $u_{\delta}$ expand (up to translations) as

$$
\left\{\begin{aligned}
u_{\delta}(\theta) & =u_{0}\left(\kappa \theta, a_{0}, k, \kappa\right)+\delta U_{1}(\theta)+\delta^{2} U_{2}(\theta)+\mathcal{O}\left(\delta^{3}\right) \\
c & =c_{0}\left(a_{0}, k, \kappa\right)+\delta^{2} c_{2}+\mathcal{O}\left(\delta^{3}\right)
\end{aligned}\right.
$$

where $u_{0}, c_{0}$ are defined as

$u_{0}\left(y, a_{0}, k, \kappa\right)=a_{0}+3 k\left(\frac{\kappa K(k)}{\pi}\right)^{2} \mathrm{cn}^{2}\left(\frac{K(k)}{\pi} y, k\right), \quad c_{0}=a_{0}+(2 k-1)\left(\frac{\kappa K(k)}{\pi}\right)^{2}$,

and $\kappa$ is determined from $k$ via the selection principle $\kappa=\mathcal{G}(k)$ with

$$
\left(\frac{K(k) \mathcal{G}(k)}{\pi}\right)^{2}=\frac{7}{20} \frac{2\left(k^{4}-k^{2}+1\right) E(k)-\left(1-k^{2}\right)\left(2-k^{2}\right) K(k)}{\left(-2+3 k^{2}+3 k^{4}-2 k^{6}\right) E(k)+\left(k^{6}+k^{4}-4 k^{2}+2\right) K(k)} .
$$

Moreover the functions $\left(U_{i}\right)_{i=1,2}$ are (respectively odd and even) solutions of the linear equations

$$
\mathcal{L}_{0}\left[U_{1}\right]+\kappa u_{0}^{\prime \prime}+\kappa^{3} u_{0}^{\prime \prime \prime \prime}=0, \quad \mathcal{L}_{0}\left[U_{2}\right]+\left(\frac{U_{1}^{2}}{2}-c_{2} u_{0}\right)^{\prime}+\kappa U_{1}^{\prime \prime}+\kappa^{3} U_{1}^{\prime \prime \prime \prime}=0,
$$

where $\mathcal{L}_{0}:=\kappa^{2} \partial_{x}^{3}+\partial_{x}\left(\left(u_{0}-c_{0}\right)\right.$.) is a closed linear operator acting on $L_{\text {per }}^{2}(0,2 K(k))$ with densely defined domain $H_{\text {per }}^{3}(0,2 K(k))$.

Remark 2.5. The condition $\kappa=\mathcal{G}(k)$ is precisely the defining relation for the period $X=2 K(k) / \kappa$ to belong to the set $\mathcal{W}$ defined in the introduction. Furthermore, the choice of $\delta_{0}$ in Proposition 2.4 can be chosen uniformly on compact subsets of $\mathcal{W}$.

Proof. The regularity of the profiles $u_{\delta}$ in $\theta$ follows by basic ODE theory, while the regularity with respect to $\delta$ follows by Proposition 2.1. Furthermore, the explicit expansions above are determined as follows. After rescaling, continuing the $2 K(k) / \kappa$-periodic wave trains of (2.1) to $0<\delta \ll 1$ is equivalent to searching for $2 K(k)$-periodic solutions of

$$
(U-c) U^{\prime}+\kappa^{2} U^{\prime \prime \prime}+\delta\left(\kappa U^{\prime \prime}+\kappa^{3} U^{\prime \prime \prime \prime}\right)=0
$$


for $\delta>0$ sufficiently small. We expand $c, u_{\delta}$ in the limit $\delta \rightarrow 0$ as

$$
c=c_{0}+\delta c_{1}+\mathcal{O}\left(\delta^{2}\right), \quad u_{\delta}(\theta)=u_{0}(\theta)+\delta U_{1}(\theta)+\mathcal{O}\left(\delta^{2}\right),
$$

with $u_{0}(\theta)=x_{0}\left(\theta, \kappa, k, a_{0}\right)$ as defined in (2.7). Notice that, up to order $\mathcal{O}(1)$, equation (2.13) is satisfied for all $a_{0}, k, \kappa$, i.e. there is no selection of a particular wave train. Now, identifying the $\mathcal{O}(\delta)$ terms in (2.13) yields the equation

$$
\kappa^{2} U_{1}^{\prime \prime \prime}+\left(\left(u_{0}-c_{0}\right) U_{1}\right)^{\prime}-c_{1} u_{0}^{\prime}+\kappa u_{0}^{\prime \prime}+\kappa^{3} u_{0}^{\prime \prime \prime \prime}=0 .
$$

The linear operator $\mathcal{L}_{0}[x]=\kappa^{2} x^{\prime \prime \prime}+\left(\left(u_{0}-c_{0}\right) x\right)^{\prime}$, defined on $H_{p e r}^{3}(0,2 K(k))$, is Fredholm of index 0 and $\left(1, u_{0}\right)$ span the kernel of its adjoint (see BrJ,JZB for more details). Then one can readily deduce that equation (2.14) has a periodic solution provided that the following compatibility condition is satisfied: $\left\langle\left(u_{0}^{\prime}\right)^{2}\right\rangle=\kappa^{2}\left\langle\left(u_{0}^{\prime \prime}\right)^{2}\right\rangle$ which is precisely the selection criterion (2.11). In order to determine $c_{1}$, one has to consider higher order corrections to $x_{0}$ : in fact, $c_{1}$ is determined through a solvability condition on the equation for $x_{2}$. This yields $c_{1}=0$ (see EMR for more details).

Remark 2.6. As indicated in Remark 2.2 while the degree of regularity of the profile $u_{\delta}$ in $\delta$ can be chosen arbitrarily, the value of $\delta_{0}$ is expected to depend on $r$. In particular, as $r \rightarrow \infty$ we expect $\delta_{0} \rightarrow 0$. Throughout the paper, we will arbitrarily choose the regularity of the profile to be sufficiently large (but finite) to make our arguments valid. This can, of course, be done at successive steps by possibly choosing the value of $\delta_{0}$ to be smaller than at the previous step, but still non-zero.

Remark 2.7. Notice that Proposition 2.4 associates to each $X \in \mathcal{W}$ a one-parameter family of $X$-periodic traveling wave solutions $\left\{u_{\delta}\right\}_{\delta \in\left[0, \delta_{0}\right)}$ with wave speeds $\left\{c_{\delta}\right\}_{\delta \in\left[0, \delta_{0}\right)}$ for some sufficiently small $\delta_{0}>0$. Throughout this paper, we will abuse notation slightly and refer to such a family simply as a periodic traveling wave solution $u_{\delta}$, defined for all $\delta \in\left[0, \delta_{0}\right)$ for some sufficiently small $\delta_{0}>0$, of (1.1), in particular taking the associated wave speeds $c$ to be implicit.

As a consequence, a two dimensional manifold is obtained of periodic traveling wave solutions (identified when coinciding up to translation) of (1.1) parametrized by $a_{0} \in \mathbb{R}$ (or alternatively $c_{0}$ ) and the wave number $\kappa \in(0,1]$ (or alternatively the parameter $k \in[0,1)$ ). As noted in Remark [1.2, it follows from the translation and Galilean invarience of (1.1) that the stability of a given periodic traveling wave solution of (1.1) depends only on the period of the wave.

\section{Stability with Respect to high-FREQUenCy PERTURbations}

In this section, we begin our study of the spectral stability of periodic traveling waves of (1.1) in the limit $\delta \rightarrow 0^{+}$. To begin, let $X \in \mathcal{W}$ and let $u_{\delta}$ be an $X$-periodic traveling wave solution of (1.1), defined for all $\delta \in\left[0, \delta_{0}\right)$ for some sufficiently small $\delta_{0}>0$. Linearizing (1.1) about $u_{\delta}$ in the co-moving frame $12(x-c t, t)=(\theta, t)$ leads to the linear evolution equation

$$
\partial_{t} v-L\left[u_{\delta}\right] v=0
$$

\footnotetext{
${ }^{12}$ Recall, by our abuse of notation, that we are suppressing the dependence of $c$ on $\delta$.
} 
governing the perturbation $v$ of $u_{\delta}$, where $L\left[u_{\delta}\right]$ denotes the differential operator with $X$-periodic coefficients

$$
L\left[u_{\delta}\right] v=-\left(\left(u_{\delta}-c\right) v\right)^{\prime}-v^{\prime \prime \prime}-\delta\left(v^{\prime \prime}+v^{\prime \prime \prime \prime}\right) .
$$

In the literature, there are many choices for the class of perturbations considered, each of which corresponds to a different domain for the above linear operator. Here, we are interested in perturbations of $U$ which are spatially localized, hence we require that $v(\cdot, t) \in L^{2}(\mathbb{R})$ for each $t \geq 0$. This naturally leads one to a detailed analysis of the spectrum of the operator $L$ considered as an operator on $L^{2}(\mathbb{R})$ with densely defined domain $H^{4}(\mathbb{R})$.

To characterize the spectrum of the operator $L\left[u_{\delta}\right]$, considered here as a densely defined operator on $L^{2}(\mathbb{R})$, we note that as the coefficients of $L\left[u_{\delta}\right]$ are $X$-periodic functions of $x$, Floquet theory implies that the spectrum of $L\left[u_{\delta}\right]$ is purely continuous and that $\lambda \in \sigma\left(L\left[u_{\delta}\right]\right)$ if and only if the spectral problem

$$
L\left[u_{\delta}\right] v=\lambda v
$$

has an $L^{\infty}(\mathbb{R})$ eigenfunction of the form

$$
v(x ; \lambda, \xi)=e^{i \xi x} w(x ; \lambda, \xi)
$$

for some $\xi \in[-\pi / X, \pi / X)$ and $w(\cdot) \in L_{\text {per }}^{2}(0, X)$. Following [G, S1, S2], we find that substituting the ansatz (3.2) into (3.1) leads one to consider the one-parameter family of Bloch operators $\left\{L_{\xi}\right\}_{\xi \in[-\pi / X, \pi / X)}$ acting on $L_{\text {per }}^{2}([0, X])$ via

$$
\left(L_{\xi}\left[u_{\delta}\right] w\right)(x):=e^{-i \xi x} L\left[u_{\delta}\right]\left[e^{i \xi \cdot} w(\cdot)\right](x) .
$$

Since the Bloch operators have compactly embedded domains $H_{\mathrm{per}}^{4}([0, X])$ in $L_{\text {per }}^{2}([0, X])$, their spectrum consists entirely of discrete eigenvalues which, furthermore, depend continuously on the Bloch parameter $\xi$. It follows by these standard considerations that

$$
\sigma_{L^{2}(\mathbb{R})}\left(L\left[u_{\delta}\right]\right)=\bigcup_{\xi \in[-\pi / X, \pi / X)} \sigma_{L_{\mathrm{per}}^{2}([0, X])}\left(L_{\xi}\left[u_{\delta}\right]\right) ;
$$

see [G] for details. As a result, the spectrum of $L$ may be decomposed into countably many curves $\lambda(\xi)$ such that $\lambda(\xi) \in \sigma\left(L_{\xi}\right)$ for $\xi \in[-\pi / X, \pi / X)$.

The spectra $\lambda$ of the Bloch operators $L_{\xi}\left[u_{\delta}\right]$ may be characterized as the zero set for fixed $\xi, \delta$ of the Evans function

$$
E(\lambda, \xi, \delta)=\operatorname{det}\left(R(X, \lambda, \delta)-e^{i \xi X} I d_{\mathbb{C}^{3}}\right),
$$

where $R(\cdot, \lambda)$ denotes the resolvent (or fundamental solution) matrix associated with the linearized eigenvalue problem (3.1) evaluated at $x=X$; that is, writing (3.1) as the first order $\operatorname{ODE} \Phi^{\prime}(x)=\mathbb{H}(x, \lambda, \delta) \Phi(x), R(\cdot, \lambda, \delta)$ is the unique solution to the initial value problem

$$
\Phi^{\prime}(x)=\mathbb{H}(x, \lambda, \delta) \Phi(x), \quad \Phi(0)=\mathrm{Id} .
$$

Thus, the spectra of $L$ consists of the union of zeros $\lambda$ as all values of $\xi \in$ $[-\pi / X, \pi / X)$ are swept out. Now, for any positive integer $r \geq 1$, Proposition 2.4 implies that we can find a $\delta_{0}>0$ such that the profile $u_{\delta}$ is a $C^{r}$ function of $\delta \in\left[0, \delta_{0}\right)$. It follows then that solutions of $L_{\xi}\left[u_{\delta}\right] v=\lambda v$ depend analytically on $(\lambda, \xi)$ on open and bounded subsets of $(\lambda, \xi) \in \mathbb{C}^{2}$, and are $C^{r}$ functions of $\delta \in\left[0, \delta_{0}\right)$. Hence, the Evans function $E$ will possess these same regularity properties. We will see in Section 3.2 that the spectral problem $E(\lambda, \xi, \delta)=0$ can be 
replaced by $\bar{E}(\lambda, \xi, \delta)=0$, equivalent to the original one for all $\delta>0$ and so that $\bar{E}$ is analytic in $(\lambda, \xi) \in \mathbb{C}^{2}$ and, for any fixed positive integer $r \geq 1$, is $C^{r}$ for all $r<\infty$ in $\delta$ for $\delta \in\left[0, \delta_{0}\right)$ provided $\delta_{0}>0$ is sufficiently small.

In the following, we will first prove that possible unstable eigenvalues are of order $\mathcal{O}(1)+i \mathcal{O}\left(\delta^{-3 / 4}\right)$ by using a standard parabolic energy estimate. By a bootstrap argument based on an approximate diagonalisation of the first order differential system associated with (3.1), we show that possible unstable eigenvalues are $\mathcal{O}(1)$, which implies that they are necessarily of order $\mathcal{O}(\delta)+i \mathcal{O}(1)$. We then provide an expansion in $\delta$ of the Evans function as $\delta \rightarrow 0$ in a bounded box close to the imaginary axis with the help of a Fenichel-type procedure and an iterative scheme based on the exact resolvent matrix associated with the linearized KdV equations.

3.1. Boundedness of unstable eigenvalues as $\delta \rightarrow 0$. In this section, we bound the region in the unstable half-plane $\Re(\lambda) \geq 0$, where the unstable essential spectrum of the linearized operator $L$ may lie in the limit $\delta \rightarrow 0$. Throughout, we use the notation $\|u\|^{2}=\int_{0}^{X}|u(x)|^{2} d x$. We begin by proving the following lemma, verifying that the unstable spectra is $\mathcal{O}\left(\delta^{-3 / 4}\right)$ for $\delta$ sufficiently small.

Lemma 3.1. Let $X \in \mathcal{W}$ and let $u_{\delta}$ be an $X$-periodic traveling wave solution of (1.1), defined for all $\delta \in\left[0, \delta_{0}\right)$ for some $\delta_{0}>0$ sufficiently small. Then there exist constants $C_{1}, C_{2}>0$ such that, for all $\delta \in\left[0, \delta_{0}\right)$, the operator $L\left[u_{\delta}\right]$ has no $L^{\infty}(\mathbb{R})$ eigenvalues with $\Re(\lambda) \geq C_{1}$ or with $\Re(\lambda) \geq 0$ and $\delta^{3 / 4}|\Im(\lambda)| \geq C_{2}$.

Proof. Suppose that $\lambda$ is an $L^{\infty}(\mathbb{R})$ eigenvalue of $L\left[u_{\delta}\right]$ and let $v$ be a corresponding eigenfunction. Multiplying equation (3.1) by $\bar{v}$ and integrating over one period, we obtain

$$
\lambda\|v\|^{2}-\int_{0}^{X}\left(\left(u_{\delta}-c\right) v+v^{\prime \prime}\right) \bar{v}^{\prime} d x+\delta\left(\left\|v^{\prime \prime}\right\|^{2}-\left\|v^{\prime}\right\|^{2}\right)=0 .
$$

Identifying the real and imaginary parts yields the system of equations:

$$
\begin{aligned}
& \Re(\lambda)\|v\|^{2}+\frac{1}{2} \int_{0}^{X} u_{\delta}^{\prime}|v|^{2} d x+\delta\left(\left\|v^{\prime \prime}\right\|^{2}-\left\|v^{\prime}\right\|^{2}\right)=0, \\
& |\Im(\lambda)|\|v\|^{2} \leq\left\|\left(u_{\delta}-c\right)\right\|_{\infty}\|v\|\left\|v^{\prime}\right\|+\left\|v^{\prime}\right\|\left\|v^{\prime \prime}\right\| .
\end{aligned}
$$

Here, we have used the fact that, by (3.2), $v(x+X)=e^{i \xi X} v(x)$ so that $|v|$ is $X$ periodic. Next, using the Sobolev estimate $\left\|v^{\prime}\right\|^{2} \leq C\|v\|^{2} / 2+\left\|v^{\prime \prime}\right\|^{2} /(2 C)$, valid for any $C>0$, into the first equation yields the bound

$$
\Re(\lambda)\|v\|^{2}+\delta\left(1-\frac{1}{2 C}\right)\left\|v^{\prime \prime}\right\|^{2} \leq \frac{1}{2}\left(\left\|u_{\delta}^{\prime}\right\|_{\infty}+\delta C\right)\|v\|^{2}, \quad C>0 .
$$

Letting $C=1 / 2$ then yields

$$
\Re(\lambda) \leq \frac{1}{2}\left(\sup _{\delta \in\left[0, \delta_{0}\right)}\left\|u_{\delta}^{\prime}\right\|_{\infty}+\frac{\delta_{0}}{2}\right),
$$

which verifies the stated bound on the real part of $\lambda$.

Suppose now $\Re(\lambda) \geq 0$. Using again the Sobolev estimate $\left\|v^{\prime}\right\| \leq\|v\|^{1 / 2}\left\|v^{\prime \prime}\right\|^{1 / 2}$, the imaginary part of $\lambda$ can be bounded as

$$
|\Im(\lambda)|\|v\|^{2} \leq\left\|u_{\delta}-c\right\|_{\infty}\|v\|^{3 / 2}\left\|v^{\prime \prime}\right\|^{1 / 2}+\|v\|^{1 / 2}\left\|v^{\prime \prime}\right\|^{3 / 2} .
$$

Furthermore, $\left\|v^{\prime \prime}\right\|$ can be controlled by

$$
\delta\left\|v^{\prime \prime}\right\|^{2} \leq\left(\left\|u_{\delta}^{\prime}\right\|_{\infty}+\delta\right)\|v\|^{2},
$$


which follows by setting $C=1$ in (3.7) and recalling that $\Re(\lambda) \geq 0$ by hypothesis. Thus, setting $K^{2}=\sup _{\delta \in\left[0, \delta_{0}\right)}\left\|u_{\delta}^{\prime}\right\|_{\infty}+\delta_{0}$ we deduce that

$$
|\Im(\lambda)| \leq\left(\sup _{\delta \in\left[0, \delta_{0}\right)}\left\|u_{\delta}-c\right\|_{\infty}\right) K^{1 / 2} \delta^{-1 / 4}+K^{3 / 2} \delta^{-3 / 4},
$$

which completes the proof.

Remark 3.2. By a slight modification, the estimates in Lemma 3.1 can be extended into the stable spectrum. Indeed, if $C_{3}>0$, then adding $C_{3}\|v\|^{2}$ to both sides of the bound (3.7) yields the estimate

$$
\left(\Re(\lambda)+C_{3}\right)\|v\|^{2}+\delta\left(1-\frac{1}{2 C}\right)\left\|v^{\prime \prime}\right\|^{2} \leq \frac{1}{2}\left(\left\|u_{\delta}^{\prime}\right\|_{\infty}+2 C_{3}+\delta C\right)\|v\|^{2} .
$$

Thus, as long as $\Re(\lambda)+C_{3} \geq 0$ we can repeat the proof on the estimates of imaginary parts to conclude

$$
|\Im(\lambda)| \leq K_{3}^{1 / 2} \delta^{-1 / 4}+K_{3} \delta^{-3 / 4}
$$

where $K_{3}=\left\|u_{\delta}^{\prime}\right\|_{\infty}+2 C_{3}+\delta$.

Next, we bootstrap the estimates in Lemma 3.1 to provide a second energy estimate on the reduced "slow", or "KdV", block of the spectral problem (3.1) in the limit $\delta \rightarrow 0$. This yields a sharper estimate on the modulus of the possibly unstable spectrum in this distinguished limit, in particular proving that unstable spectra must lie in a compact region in the complex plane. Notice that this result relies heavily on the fact that the corresponding spectral problem for the linearized $\mathrm{KdV}$ equation about a cnoidal wave (2.7) has been explicitly solved in $\mathrm{BD}, \mathrm{Sp}$.

Proposition 3.3. Let $X \in \mathcal{W}$ and let $u_{\delta}$ be an $X$-periodic traveling wave solution of (1.1), defined for all $\delta \in\left[0, \delta_{0}\right)$ for some $\delta_{0}>0$ sufficiently small. Then there exists a constant $0<\delta^{*}<\delta_{0}$ and constants $C_{1}, C_{2}>0$ such that for all $\delta \in\left[0, \delta^{*}\right)$ the operator $L\left[u_{\delta}\right]$ has no $L^{\infty}(\mathbb{R})$ eigenvalues with $\Re(\lambda) \geq 0$ and either $\Re(\lambda) \geq C_{1} \delta$ or $|\Im(\lambda)| \geq C_{2}$.

Proof. The proof is done in two steps: first, we show that if $\lambda$ is an $L^{\infty}(\mathbb{R})$ eigenvalue of $L\left[u_{\delta}\right]$ with $\Re(\lambda) \geq 0$ and corresponding eigenfunction $v$, then there exists $C_{2}>0$ such that $|\Im(\lambda)| \leq C_{2}$. The estimate on $\Re(\lambda)$ will then easily follow. To begin, let $r \geq 3$ be a positive integer, and let $\delta_{1}>0$ be such that the profiles $u_{\delta}$ constructed in Proposition 2.4 are $C^{r}$ functions of $\delta \in\left[0, \delta_{1}\right)$. Next, let $(v, \lambda)$ be an $L^{\infty}(\mathbb{R})$ eigenpair of (3.1) with $\Re(\lambda) \geq 0$ and set $x=v, y=v^{\prime}, z=v^{\prime \prime}+v, w=z^{\prime}$, and $s=c+1$, so that (3.1) may be written as the first order system

$$
x^{\prime}=y, \quad y^{\prime}=z-x, \quad z^{\prime}=w, \quad \delta w^{\prime}=-w-\left(u_{\delta}^{\prime}+\lambda\right) x-\left(u_{\delta}-s\right) y .
$$

We first apply a Fenichel-type procedure and introduce $w_{1}=w+\left(u_{\delta}^{\prime}+\lambda\right) x+\left(u_{\delta}-s\right) y$, noting then that $w_{1}$ satisfies

$$
\delta w_{1}^{\prime}=-w_{1}+\delta\left(u_{\delta}^{\prime \prime} x+\left(2 u_{\delta}^{\prime}+\lambda\right) y+\left(u_{\delta}-s\right)(z-x)\right) .
$$


We further introduce $w_{2}=w_{1}-\delta\left(u_{\delta}^{\prime \prime} x+\left(2 u_{\delta}^{\prime}+\lambda\right) y+\left(u_{\delta}-s\right)(z-x)\right)$ so that $w_{2}$ satisfies the equation

$$
\begin{aligned}
& \delta w_{2}^{\prime}=-\left(1+\delta^{2}\left(u_{\delta}-s\right)\right) w_{2}-\delta^{2}\left(\left(u_{\delta}^{\prime \prime \prime}-\left(u_{\delta}-s\right)\left(u_{\delta}^{\prime}+\lambda-\delta u_{\delta}^{\prime \prime}\right)\right) x\right. \\
&-\delta^{2}\left(\left(3 u_{\delta}^{\prime \prime}-\left(u_{\delta}-s\right)^{2}+\delta\left(u_{\delta}-s\right)\left(2 u_{\delta}^{\prime}+\lambda\right)\right) y\right. \\
&\left.+\left(3 u_{\delta}^{\prime}+\lambda+\delta\left(u_{\delta}-s\right)^{2}\right)(z-x)\right) .
\end{aligned}
$$

Now, by Lemma 3.1 we know that necessarily one has $\Re(\lambda)+\delta^{3 / 4}|\Im(\lambda)| \leq C$ for some constant $C>0$. It follows that $\lambda \delta=o(1)$ as $\delta \rightarrow 0$; hence, for $\delta \geq 0$ sufficiently small we may rewrite system (3.8) as

$$
\begin{aligned}
x^{\prime} & =y, \quad y^{\prime}=z-x \\
z^{\prime} & =w_{2}-\left(u_{\delta}^{\prime}+\lambda\right) x-\left(u_{\delta}-s\right) y+\delta\left(u_{\delta}^{\prime \prime} x+\left(2 u_{\delta}^{\prime}+\lambda\right) y+\left(u_{\delta}-s\right)(z-x)\right), \\
\delta w_{2}^{\prime} & =-w_{2}-\delta^{2} \lambda\left((z-x)-\left(u_{\delta}-s\right) x\right)+\mathcal{O}\left(\delta^{2}\left(|x|+|y|+|z|+\left|w_{2}\right|\right)\right) .
\end{aligned}
$$

Next, we remove $w_{2}$ from the equation in $z$ by introducing the variable $z_{*}=z+\delta w_{2}$, in terms of which (3.9) reads for $\delta \geq 0$ sufficiently small

$$
\begin{gathered}
x^{\prime}=y, \quad y^{\prime}=z_{*}-\delta w_{2}-x \\
z_{*}^{\prime}=-\left(u_{\delta}^{\prime}+\lambda\right) x-\left(u_{\delta}-s\right) y+\delta\left(u_{\delta}^{\prime \prime} x+\left(2 u_{\delta}^{\prime}+\lambda\right) y+\left(u_{\delta}-s\right)\left(z_{*}-x\right)\right) \\
\quad-\delta^{2} \lambda\left(\left(z_{*}-x\right)-\left(u_{\delta}-s\right) x\right)+\mathcal{O}\left(\delta^{2}\left(|x|+|y|+\left|z_{*}\right|+\left|w_{2}\right|\right)\right), \\
\delta w_{2}^{\prime}=-w_{2}-\delta^{2} \lambda\left((\bar{z}-x)-\left(u_{\delta}-s\right) x\right)+\mathcal{O}\left(\delta^{2}\left(|x|+|y|+\left|z_{*}\right|+\left|w_{2}\right|\right)\right) .
\end{gathered}
$$

We further introduce the variables $\bar{y}=y-\delta^{2} w_{2}, \bar{x}=x$ and $\bar{z}=z_{*}-\bar{x}$. For $\delta$ sufficiently small the system (3.10) then reads

$$
\begin{gathered}
\bar{x}^{\prime}=\bar{y}+\mathcal{O}\left(\delta^{2}\left(|\bar{x}|+|\bar{y}|+|\bar{z}|+\left|w_{2}\right|\right)\right), \quad \bar{y}^{\prime}=\bar{z}+\mathcal{O}\left(\delta^{2}\left(|\bar{x}|+|\bar{y}|+|\bar{z}|+\left|w_{2}\right|\right)\right), \\
\bar{z}^{\prime}=-\left(u_{\delta}^{\prime}+\lambda\right) \bar{x}-\left(u_{\delta}-c\right) \bar{y}+\delta\left(u_{\delta}^{\prime \prime} \bar{x}+\left(2 u_{\delta}^{\prime}+\lambda\right) \bar{y}+\left(u_{\delta}-s\right) \bar{z}\right) \\
\quad-\delta^{2} \lambda\left(\bar{z}-\left(u_{\delta}-s\right) \bar{x}\right)+\mathcal{O}\left(\delta^{2}\left(|\bar{x}|+|\bar{y}|+|\bar{z}|+\left|w_{2}\right|\right)\right), \\
\delta w_{2}^{\prime}=-w_{2}-\delta^{2} \lambda\left(\bar{z}-\left(u_{\delta}-s\right) \bar{x}\right)+\mathcal{O}\left(\delta^{2}\left(|\bar{x}|+|\bar{y}|+|\bar{z}|+\left|w_{2}\right|\right)\right) .
\end{gathered}
$$

In particular, by neglecting $\mathcal{O}(\delta)$ terms in the $(\bar{x}, \bar{y}, \bar{z})$ equations in (3.11) and eliminating $(\bar{y}, \bar{z})$, we recognize the spectral problem associated with the linearized $\mathrm{KdV}$ equation about the periodic wave $u_{0}$ with speed $c_{0}$ :

$$
\bar{x}^{\prime \prime \prime}+\left(\left(u_{0}-c_{0}\right) \bar{x}\right)^{\prime}+\lambda \bar{x}=0 .
$$

The above calculations motivate us to make a reduction to the "KdV block" of the spectral problem (3.1). More precisely, recalling (2.12), we write the differential system (3.11 $),\left(3.11_{b}\right),\left(3.11_{3}\right)$ on $\bar{X}=(\bar{x}, \bar{y}, \bar{z})^{T}$ as

$$
\bar{X}^{\prime}=\left(A_{0}+\delta\left(A_{1}+\lambda A_{2}\right)+\lambda \delta^{2} A_{3}+\mathcal{O}\left(\delta^{2}\right)\right) \bar{X}+\mathcal{O}\left(\delta^{2}\left|w_{2}\right|\right),
$$


where

$$
A_{0}=\left(\begin{array}{ccc}
0 & 1 & 0 \\
0 & 0 & 1 \\
-\left(u_{0}^{\prime}+\lambda\right) & -\left(u_{0}-c_{0}\right) & 0
\end{array}\right)
$$

denotes the coefficient matrix for the linearized $\mathrm{KdV}$ equation, and $A_{1}, A_{2}$, and $A_{3}$ are defined as

$$
\begin{gathered}
A_{1}=\left(\begin{array}{ccc}
0 & 0 & 0 \\
0 & 0 & 0 \\
-U_{1}^{\prime}+u_{0}^{\prime \prime} & -U_{1}+2 u_{0}^{\prime} & u_{0}-c_{0}
\end{array}\right), \\
A_{2}=\left(\begin{array}{lll}
0 & 0 & 0 \\
0 & 0 & 0 \\
0 & 1 & 0
\end{array}\right), A_{3}=\left(\begin{array}{ccc}
0 & 0 & 0 \\
0 & 0 & 0 \\
u_{0}-c_{0} & 0 & -1
\end{array}\right) .
\end{gathered}
$$

In order to analyze (3.12) for $0<\delta \ll 1$, we recall that in $\mathrm{BD}$ the complete integrability of (2.1) was used to determined a basis of solutions of $X^{\prime}=A_{0} X$, at least when $\lambda \neq 0$, which corresponds to a linearized KdV equation about the periodic wave train $U_{0}$. Specifically, such a basis $\left(V_{i}\right)_{i=1,2,3}$ is defined as $V_{i}=$ $\left(\hat{u}_{i}, \hat{u}_{i}^{\prime}, \hat{u}_{i}^{\prime \prime}\right)$ with $\hat{u}_{i}$ given by

$$
\hat{u}_{i}(\theta, \lambda)=\left(1-\frac{u_{0}^{\prime}}{3 \lambda}\right) \exp \left(\int_{0}^{\theta} \frac{\lambda d y}{u_{0}(y) / 3-c_{0}+\eta_{i}}\right),
$$

and $\eta_{i}$ are solutions of the polynomial equation

$$
\left(\eta-4 \xi_{1}\right)\left(\eta-4 \xi_{2}\right)\left(\eta-4 \xi_{3}\right)=\lambda^{2},
$$

where $\xi_{1}=k^{2}-1, \xi_{2}=2 k^{2}-1, \xi_{3}=k^{2}$. In order to deal with the limit $|\lambda| \rightarrow \infty$, we introduce the diagonal matrix $D(\lambda)$ with

$$
D_{i i}(\lambda)=-\left\langle\frac{\lambda}{u_{0} / 3-c_{0}+\eta_{i}}\right\rangle,
$$

where $\langle g(\cdot)\rangle$ denotes the average of the function $g$ over $[0, X]$ and write a resolvent matrix for $X^{\prime}=A_{0} X$ as

$$
R(\lambda, \theta)=P(\lambda, \theta) e^{D(\lambda) \theta},
$$

where $P(\lambda, \theta)=\left(\bar{V}_{1}, \bar{V}_{2}, \bar{V}_{3}\right)(\lambda, \theta)$ is the matrix function with columns being given by the vector valued functions $\bar{V}_{k, i}(\lambda, \theta)=e^{-D_{k k}(\lambda) \theta} \partial_{\theta}^{(i-1)} \hat{u}_{k}(\theta), i=1,2,3$. Next we make the periodic change of variable $\bar{X}(\theta)=P(\lambda, \theta) Y(\theta)$, which is nothing but the classical change of variable in Floquet's theorem. In terms of $Y$, system (3.12) expands as

$$
Y^{\prime}=\left(D(\lambda)+\delta P^{-1}\left(A_{1}+\lambda A_{2}+\lambda \delta A_{3}+\mathcal{O}(\delta)\right) P\right) Y+\mathcal{O}\left(\delta^{2}\left\|P^{-1}\right\|\left|w_{2}\right|\right)
$$

as $|\lambda| \rightarrow \infty$.

We now analyze the individual terms in (3.15) more closely. To this end, first notice that as $|\lambda| \rightarrow \infty$ the eigenfunctions associated with the linearized KdV equation expand as

$$
\hat{u}_{i}(\theta, \lambda)=\left(1+\mathcal{O}\left(|\lambda|^{-1 / 3}\right)\right) e^{D_{i i}(\lambda) \theta} .
$$


It follows that as $|\lambda| \rightarrow \infty$ the matrix $P$ defined above expands as

$$
P=\left(\begin{array}{ccc}
1 & 1 & 1 \\
\Lambda_{1} & \Lambda_{2} & \Lambda_{3} \\
\Lambda_{1}^{2} & \Lambda_{2}^{2} & \Lambda_{3}^{2}
\end{array}\right)\left(1+\mathcal{O}\left(|\lambda|^{-1 / 3}\right),\right.
$$

where $\Lambda_{i}:=D_{i i}(\lambda)$. Thus, by a straightforward calculation we see that as $|\lambda| \rightarrow \infty$ we have the estimates $\|P(\lambda, \cdot)\|_{L^{\infty}(\mathbb{R})}=\mathcal{O}\left(|\lambda|^{2 / 3}\right)$ and

$$
\begin{gathered}
\left\|P^{-1}(\lambda, \cdot)\right\|_{L^{\infty}(\mathbb{R})}=\mathcal{O}(1), \quad\left\|P^{-1} A_{1} P\right\|_{L^{\infty}(\mathbb{R}, d \theta)}=\mathcal{O}(1), \\
\left\|P^{-1} A_{3} P\right\|_{L^{\infty}(\mathbb{R}, d \theta)}=\mathcal{O}(1) .
\end{gathered}
$$

Hence, using the fact that $|\lambda| \delta^{3 / 4}=\mathcal{O}(1)$, equation (3.15) can be rewritten as

$$
Y^{\prime}=\left(D(\lambda)+\delta\left(\lambda P^{-1} A_{2} P+\mathcal{O}(1)\right)+\mathcal{O}\left(\delta^{5 / 4}\right)\right) Y+\mathcal{O}\left(\delta^{2}\left|w_{2}\right|\right) .
$$

Finally, with a near-identity change of variables of the form $\tilde{Y}=\left(I d+\mathcal{O}\left(\delta|\lambda|^{1 / 3}\right)\right) Y$ one can remove the non-diagonal part of $\delta\left(\lambda P^{-1} A_{2} P+\mathcal{O}(1)\right)$ up to $\mathcal{O}\left(\delta^{5 / 4}\right)$ so that (3.16) reads

$$
\tilde{Y}^{\prime}=\left(D(\lambda)+\delta \operatorname{diag}\left(\lambda P^{-1} A_{2} P+\mathcal{O}(1)\right)+\mathcal{O}\left(\delta^{5 / 4}\right)\right) \tilde{Y}+\mathcal{O}\left(\delta^{2}\left|w_{2}\right|\right) .
$$

Next, define the diagonal matrix $\Gamma(\lambda):=D(\lambda)+\delta \operatorname{diag}\left(\lambda P^{-1} A_{2} P+\mathcal{O}(1)\right)$ with diagonal entries

$$
\begin{aligned}
& \Gamma(\lambda)_{11}=\Lambda_{1}+\delta\left(\lambda \frac{\Lambda_{1}\left(\Lambda_{3}-\Lambda_{2}\right)}{\Delta}+\mathcal{O}\left(|\lambda|^{1 / 3}\right)\right), \\
& \Gamma(\lambda)_{22}=\Lambda_{2}+\delta\left(\lambda \frac{\Lambda_{2}\left(\Lambda_{1}-\Lambda_{3}\right)}{\Delta}+\mathcal{O}\left(|\lambda|^{1 / 3}\right)\right), \\
& \Gamma(\lambda)_{33}=\Lambda_{3}+\delta\left(\lambda \frac{\Lambda_{3}\left(\Lambda_{2}-\Lambda_{1}\right)}{\Delta}+\mathcal{O}\left(|\lambda|^{1 / 3}\right)\right),
\end{aligned}
$$

where $\Delta:=\left(\Lambda_{2}-\Lambda_{1}\right)\left(\Lambda_{3}-\Lambda_{1}\right)\left(\Lambda_{3}-\Lambda_{2}\right)$. From (3.14) it follows that $\eta_{i}=\mathcal{O}\left(|\lambda|^{2 / 3}\right)$ as $|\lambda| \rightarrow \infty$, from which we see $\Lambda_{i}(\lambda)=\mathcal{O}\left(|\lambda|^{1 / 3}\right)$ in this limit. Introducing the polar coordinates $\lambda=|\lambda| e^{i(\pi / 2-\phi)}$, and noting that $\Re(\lambda)=\mathcal{O}(1)$ by Lemma 3.1 . we find that $\phi=\mathcal{O}\left(|\lambda|^{-1}\right)$ as $|\lambda| \rightarrow \infty$. Directly expanding the $D_{i i}(\lambda)$, we have

$$
\Lambda_{1}=|\lambda|^{1 / 3} e^{i(\pi / 2-\phi / 3)}+\mathcal{O}\left(\lambda^{-1 / 3}\right), \quad \Lambda_{2}=j \Lambda_{1}+\mathcal{O}(1), \quad \Lambda_{3}=j^{2} \Lambda_{1}+\mathcal{O}(1),
$$

where $j=e^{2 \pi i / 3}$ denotes the principal third root of unity so that, in particular, we have the estimates

$$
\Re\left(\Lambda_{2}\right)=\frac{\sqrt{3}}{2}|\lambda|^{1 / 3}+\mathcal{O}(1), \quad \Re\left(\Lambda_{3}\right)=-\frac{\sqrt{3}}{2}|\lambda|^{1 / 3}+\mathcal{O}(1)
$$

as $|\lambda| \rightarrow \infty$.

With the above preparations, we are now in a position to perform the necessary energy estimates. Indeed, under the condition $\tilde{Y}(x+X)=e^{i \gamma} \tilde{Y}(x)$ and $w_{2}(x+X)=$ $e^{i \gamma} w_{2}(x)$ and recalling that $\|P(\lambda, \cdot)\|_{L^{\infty}(\mathbb{R})}=\mathcal{O}\left(|\lambda|^{2 / 3}\right)$, it follows from (3.11) that

$$
\left\|w_{2}\right\| \leq C|\lambda| \delta^{2}\|P(\lambda, \cdot)\|_{\infty}(\|\tilde{x}\|+\|\tilde{y}\|+\|\tilde{z}\|) \leq C \delta^{3 / 4}(\|\tilde{x}\|+\|\tilde{y}\|+\|\tilde{z}\|),
$$


where here we set $\tilde{Y}=(\tilde{x}, \tilde{y}, \tilde{z})^{T}$. Similarly, using the bounds in (3.18), it follows from (3.17) that

$$
\|\tilde{y}\|+\|\tilde{z}\| \leq C \frac{\delta^{5 / 4}}{|\lambda|^{1 / 3}}\|\tilde{x}\| .
$$

Inserting the bounds (3.19) and (3.20) into the $\tilde{x}$ equation in (3.17) and recalling that the function $\tilde{x}$ must be uniformly bounded on $\mathbb{R}$ as a function of $\theta$, we find necessarily that $\Re\left(\Gamma(\lambda)_{11}\right)=\mathcal{O}\left(\delta^{5 / 4}\right)$ as $|\lambda| \rightarrow \infty$, i.e. we have

$$
\Re\left(\Lambda_{1}\right)+\delta\left(\frac{|\lambda|^{2 / 3}}{3}+\mathcal{O}\left(|\lambda|^{1 / 3}\right)\right)=\mathcal{O}\left(\delta^{5 / 4}\right)
$$

which, as $|\lambda| \rightarrow \infty$, reduces to

$$
0 \leq \frac{\Re(\lambda)}{|\lambda|^{2 / 3}}\left(1+\mathcal{O}\left(|\lambda|^{-1 / 3}\right)\right)+\delta\left(|\lambda|^{2 / 3}+\mathcal{O}\left(|\lambda|^{1 / 3}\right)\right) \leq C \delta^{5 / 4}
$$

Since we have assumed $\Re(\lambda) \geq 0$, it immediately follows that $|\lambda|$ must indeed be bounded. More precisely, we deduce that there exists $C_{2}$ and $\delta_{1}>0$ such that for all $0<\delta<\delta_{1}$, the operator $L$ has no unstable eigenvalues $\lambda$ on $L^{\infty}(\mathbb{R})$ such that $|\lambda|>C_{2}$. As we have already verified in Lemma 3.1 that $\Re(\lambda)$ is necessarily bounded, we obtain a uniform bound on $|\Im(\lambda)|$. Moreover, it is then easy to show, by using (3.21), that, necessarily, possible unstable eigenvalues satisfy $0 \leq \Re(\lambda) \leq$ $C \delta$ for some constant $C>0$, and the proposition is proved.

Remark 3.4. As discussed in Remark 3.2, the estimate $|\Im(\lambda)|=\mathcal{O}\left(\delta^{-3 / 4}\right)$ is actually valid so long as $\Re(\lambda)=\mathcal{O}(1)$. Thus, by repeating the argument of Proposition 3.3 . one can prove that for any $C>0$ there exists $M, \delta_{1}>0$ such that if $0 \leq \delta \leq \delta_{1}$ and $|\lambda| \geq M$, then there are no eigenvalues $\lambda$ such that $\Re(\lambda) \geq-C \delta$.

As a result of Proposition 3.3 and Remark 3.4, we have proved the following corollary.

Corollary 3.5. Let $X \in \mathcal{W}$ and let $u_{\delta}$ be an $X$-periodic traveling wave solution of (1.1), defined for all $\delta \in\left[0, \delta_{0}\right)$ for some $\delta_{0}>0$ sufficiently small. Then given any constant $C>0$, there exist constants $M>0$ and $\delta_{1} \in\left(0, \delta_{0}\right)$ such that for $0 \leq \delta<\delta_{1}$ we have

$$
\sigma_{L^{2}(\mathbb{R})}\left(L\left[u_{\delta}\right]\right) \subset\{\lambda \in \mathbb{C} \mid \Re(\lambda) \leq-C \delta\} \cup\{\lambda \in \mathbb{C}|| \Re(\lambda)|\leq C \delta,| \Im(\lambda) \mid \leq M\} .
$$

In summary, we have restricted the location of the unstable part of the $L^{2}(\mathbb{R})$ spectrum of the linearized operator $L\left[u_{\delta}\right]$ to a compact subset of $\mathbb{C}$, uniformly for $\delta$ sufficiently small. Our next goal is to prove convergence, for a fixed $\xi$, of the eigenvalues of the Bloch operator $L_{\xi}$ to the eigenvalues of the linearized KdV equation as $\delta \rightarrow 0$. This is accomplished in the next section through the use of the periodic Evans function.

Remark 3.6. The structure of the argument of Proposition 3.3 may be recognized as somewhat similar to those of arguments used in [JZ2, PZ, HLZ, BHZ to treat other delicate limits in asymptotic ODE. A new aspect here is the incorporation of detailed estimates on the limiting system afforded by complete integrability of $\mathrm{KdV}$, which appear to be crucial in obtaining the final result. 
3.2. Expansion of the Evans function as $\delta \rightarrow 0$. In this section, we provide an expansion of both the Evans function and eigenvalues in the vicinity of the imaginary axis where all the eigenvalues are located at $\delta=0$ (this is the spectral stability result of $\mathrm{BD}, \mathrm{Sp}])$. To this end, we will use the basis of solutions constructed in BD] to build an approximation of the resolvent matrix associated with the full spectral problem (3.1). This leads us to the following result.

Proposition 3.7. Let $X \in \mathcal{W}$ and let $u_{\delta}$ be an $X$-periodic traveling wave solution of (1.1), defined for all $\delta \in\left[0, \delta_{0}\right)$ for some $\delta_{0}>0$ sufficiently small. Then on any compact set $\lambda \in K \subset \mathbb{C}$, the Evans function (3.4) of the associated spectral problem (3.1) can be expanded for $0<\delta \ll 1$ as

$$
\begin{aligned}
& E(\lambda, \delta, \xi)=-e^{i \xi X}(1+\delta e(\lambda, \xi, \delta)) \exp \left(\frac{X}{\delta}\right) \bar{E}(\lambda, \delta, \xi), \\
& \bar{E}(\lambda, \xi, \delta)=E_{\mathrm{KdV}}(\lambda, \xi)+\delta E_{1}(\lambda, \xi)+\delta^{2} E_{2}(\lambda, \xi, \delta),
\end{aligned}
$$

with $E_{\mathrm{KdV}}(\lambda, \xi)=\operatorname{det}\left(R_{\mathrm{KdV}}(X, \lambda)-e^{i \xi X} I_{\mathbb{C}^{3}}\right), R_{\mathrm{KdV}}(\cdot, \lambda)$ being the resolvent matrix associated with the linearized $K d V$ equation. Furthermore, given any positive integer $r \geq 1$, there exists a $\delta_{1} \in\left(0, \delta_{0}\right)$ sufficiently small such that the functions $e$ and $\bar{E}$ are analytic with respect to $(\lambda, \xi) \in K \times[-\pi / X, \pi / X)$ and $C^{r}$ with respect to $\delta \in\left[0, \delta_{1}\right)$. Hence, the spectral problem $\bar{E}(\lambda, \xi, \delta)=0$ is equivalent to $E(\lambda, \xi, \delta)=0$ for $\delta$ sufficiently small. Moreover for each fixed Bloch wave number $\xi \in[-\pi / X, \pi / X)$ and $\delta$ sufficiently small, if an eigenvalue $\left(\lambda_{\delta}(\xi)\right)$ of $L_{\xi}\left[u_{\delta}\right]$, defined for $\delta$ sufficiently small, belongs to $K$ for all $\delta$ sufficiently small, then $\lambda_{\delta}(\xi)$ converges to $\lambda_{0}(\xi)$, an eigenvalue of the linearized $K d V$ equation, as $\delta \rightarrow 0$, uniformly with respect to $\xi$.

Proof. To begin, let $r \geq 1$ be a fixed positive integer, and let $\delta_{0}>0$ be small enough that the profiles $u_{\delta}$ exist and depend in a $C^{r+5}$ on $\delta \in\left[0, \delta_{0}\right)$. First, we carry out a Fenichel-type computation on the spectral problem (3.1) up to $\mathcal{O}\left(\delta^{2}\right)$. Recall that by Corollary 3.5 the $L^{\infty}(\mathbb{R})$ unstable eigenvalues of the operator $L\left[u_{\delta}\right]$ are uniformly bounded in $\mathbb{C}$. Redoing the computations done in the proof of Proposition 3.3 the spectral problem (3.1) is transformed into the system

$$
\begin{aligned}
\bar{x}^{\prime}= & \bar{y}+\delta^{2} f_{1}\left(\theta, \bar{x}, \bar{y}, \bar{z}, w_{2}, \lambda, \delta\right), \\
\bar{y}^{\prime}= & \bar{z}+\delta^{2} f_{2}\left(\theta, \bar{x}, \bar{y}, \bar{z}, w_{2}, \lambda, \delta\right), \\
\bar{z}^{\prime}=- & \left(u_{0}^{\prime}+\lambda\right) \bar{x}-\left(u_{0}-c\right) \bar{y}+\delta\left(\left(u_{0}^{\prime \prime}-U_{1}^{\prime}\right) \bar{x}+\left(2 u_{0}^{\prime}-U_{1}+\lambda\right) \bar{y}+\left(u_{0}-s\right) \bar{z}\right) \\
& \quad+\delta^{2} f_{3}\left(\theta, \bar{x}, \bar{y}, \bar{z}, w_{2}, \lambda, \delta\right), \\
\delta w_{2}^{\prime}=- & w_{2}+\delta^{2} f_{4}\left(\theta, \bar{x}, \bar{y}, \bar{z}, w_{2}, \lambda, \delta\right),
\end{aligned}
$$

where the $f_{i}, i=1, \ldots, 4$, are analytic functions of $\theta \in \mathbb{R}$, linear functions in $(\bar{x}, \bar{y}, \bar{z})$, polynomial in $\lambda$, and $C^{r+3}$ functions of $\delta \in\left[0, \delta_{0}\right)$. Introducing $Y=$ $(\bar{x}, \bar{y}, \bar{z})$, we can thus write (3.23) as

$Y^{\prime}=A(\theta, \delta, \lambda) Y+\delta^{2} w_{2} F(\theta, \lambda, \delta), \quad \delta w_{2}^{\prime}=-\left(1+\delta^{2} B(\theta, \delta, \lambda)\right) w_{2}+\delta^{2} G(\theta, \lambda, \delta)^{T} Y$, where the functions $F, G, A, B$ are analytic functions of $\theta \in \mathbb{R}$, analytic functions of $\lambda \in \mathbb{C}$, and $C^{r+3}$ functions of $\delta \in\left[0, \delta_{0}\right)$.

Further, we denote by $R(\cdot ; \lambda, \delta)$ the resolvent matrix associated to $Y^{\prime}=A(. ; \delta, \lambda) Y$. It is a clear consequence of the regularity of the flow associated with this latter differential system that $R(\cdot ; \lambda, \delta)$ is analytic with respect to $\lambda \in \mathbb{C}$ and $C^{r+3}$ with 
respect to $\delta \in\left[0, \delta_{0}\right)$. Moreover $R$ expands as $R(\cdot, \lambda, \delta)=R_{\mathrm{KdV}}(\cdot, \lambda)+\delta R_{1}(\cdot ; \lambda, \delta)$, where $R_{\mathrm{KdV}}$ is the resolvent matrix of the linearized $\mathrm{KdV}$ equation $Y^{\prime}=A(. ; 0, \lambda) Y$ satisfying the initial condition $R_{\mathrm{KdV}}(0, \lambda)=I d_{\mathbb{C}^{3}}$. In order to simplify the notation in the forthcoming calculations, we now drop the $(\lambda, \delta)$ dependence of resolvent matrices.

Next, we seek to construct a basis of solutions of (3.24) valid for $0<\delta \ll 1$. To this end, notice that by Duhamel's formula the system (3.24) can be equivalently written as

$$
\begin{aligned}
& Y(\theta)=R(\theta) Y(0)+\delta^{2} \int_{0}^{\theta} w_{2}(\eta) R(\theta) R^{-1}(\eta) F(\eta) d \eta \\
& w_{2}(\theta)=\exp \left(-\int_{0}^{\theta} \mu(q) d q\right) w_{2}(0)+\delta \int_{0}^{\theta} \exp \left(-\int_{\eta}^{\theta} \mu(q) d q\right) G^{T}(\eta) Y(\eta) d \eta,
\end{aligned}
$$

where here $\mu(q)=\delta^{-1}+\delta B(q ; \lambda, \delta)$. As a first step, we build a set of 3 eigenvectors which are continuations of the eigenvectors of the linearized $\mathrm{KdV}$ equation. For that purpose, we set $w_{2}(0)=0$ and write $Y$ as

$Y(\theta)=R(\theta) Y(0)+\delta^{3} \int_{0}^{\theta} \int_{0}^{\eta} \exp \left(-\int_{\zeta}^{\eta} \mu(q) d q\right) G^{T}(\zeta) Y(\zeta) R(\theta) R^{-1}(\eta) F(\eta) d \zeta d \eta$.

By applying a fixed point argument in $C^{0}\left([0, X] ; \mathbb{C}^{3}\right)$ to 3.25 and for $\delta$ sufficiently small, we find a set of three eigenvectors $\left(Y_{i}, w_{2, i}\right)_{i=1,2,3}$ of (3.24) given by $Y_{i}=R(\xi) e_{i}+\mathcal{O}\left(\delta^{3}\right)$ with $e_{i, j}=\delta_{i, j}$ and $w_{2, i}=\mathcal{O}(\delta)$. To find a fourth linearly independent eigenvector of (3.24), we seek a solution $\left(Y, w_{2}\right)=\left(Y_{4}, w_{2,4}\right)$ such that

$$
w_{2}=\exp \left(\int_{\theta}^{X} \mu(q) d q\right)\left(1+\delta \int_{0}^{\theta} \exp \left(-\int_{\eta}^{X} \mu(q) d q\right) G^{T}(\eta) Y(\eta) d \eta\right)
$$

in particular, notice then that $w_{2}(0) \neq 0$. Then choosing $Y(0)$ so that $Y(X)=0$ gives

$$
\begin{gathered}
\exp \left(-\int_{\theta}^{X} \mu(q) d q\right) Y(\theta)=-\delta^{2} \int_{\theta}^{X} \exp \left(-\int_{\eta}^{\theta} \mu(q) d q\right) R(\theta) R^{-1}(\eta) F(\eta) d \zeta d \eta \\
+\delta \int_{0}^{\eta} \exp \left(-\int_{\zeta}^{X} \mu(q) d q\right) G(\zeta) Y(\zeta) R(\theta) R^{-1}(\eta) F(\eta) d \zeta d \eta .
\end{gathered}
$$

We then apply a fixed point argument in weighted space

$$
\exp \left(-\int_{\omega}^{X} \mu(q) d q\right) C^{0}\left([0, X] ; \mathbb{C}^{3}\right)
$$

to (3.27) to obtain a solution $Y_{4}$ such that

$$
\begin{aligned}
& \exp \left(-\int_{\theta}^{X} \mu(q) d q\right) Y_{4}(\theta) \\
& \quad=-\delta^{2} \int_{\theta}^{X} \exp \left(-\int_{\eta}^{\xi} \mu(q) d q\right) R(\xi) R^{-1}(\eta) F(\eta) d \eta+\mathcal{O}\left(\delta^{3}\right) .
\end{aligned}
$$

By plugging $Y_{4}(\theta)$ into (3.26), one completes the basis of solutions of (3.24) for $0<\delta \ll 1$. 
Let us now consider the regularity of the basis of solution $\left(Y_{i}, w_{i}\right)_{i=1, \cdots, 4}$. We start with $\left(Y_{j}, w_{j}\right)_{j=1,2,3}$. By applying the fixed point procedure in $C^{0}([0, X] ; \mathcal{H}(\Omega))$ (where $\mathcal{H}(\Omega)$ is the set of holomorphic functions on $\Omega \subset K$ an open set), one proves that for all $\delta \in\left(0, \delta_{0}\right),\left(Y_{j}, w_{j}\right)_{j=1,2,3}$ are analytic in $\theta \in \mathbb{R}$ and are holomorphic, and thus analytic, functions with respect to $\lambda \in K$, and are $C^{r}$ functions of $\delta \in\left[0, \delta_{0}\right)$.

The regularity with respect to $\delta \in\left[0, \delta_{0}\right)$ comes as follows. First, for $j=1,2,3$ it is clear that the estimates $Y_{j}=R(\theta) e_{j}+\mathcal{O}\left(\delta^{3}\right)$ and $w_{j}=\mathcal{O}(\delta)$ easily yield the continuity of $\left(Y_{j}, w_{j}\right)_{j=1,2,3}$ at $\delta=0$. Next, recalling that the profiles $U=U_{\delta}$ are assumed to be $C^{r+5}$ in $\delta \in\left[0, \delta_{0}\right)$, we find by differentiating the fixed point relation (3.25) with respect to $\delta$ and applying a fixed point argument in $C^{0}\left([0, X] ; \mathbb{C}^{3}\right)$ for $\partial_{\delta} Y_{j}, j=1,2,3$, that $\partial_{\delta} Y_{j} \in C^{0}\left([0, X] ; \mathbb{C}^{3}\right)$ for all $\delta \in\left[0, \delta_{0}\right)$ and that

$$
\partial_{\delta} Y_{j}=\partial_{\delta} R(\theta) e_{j}+\mathcal{O}\left(\delta^{2}\right) .
$$

It follows that for $j=1,2,3$ the functions $\partial_{\delta} Y_{j}$ are continuous at $\delta=0$, and hence the functions $Y_{j}, j=1,2,3$, are $C^{1}$ in $\delta$ at $\delta=0$. The continuity of $\partial_{\delta} w_{j}$ at $\delta=0$ then immediately follows 13 To now prove $C^{2}$ regularity in $\delta$ for the functions $Y_{j}, j=1,2,3$, one must first carry our one additional Fenichel-type step in the transformations carried out in Proposition 3.3 as to obtain $\mathcal{O}\left(\delta^{3}\right)$ remainders in a system of the form (3.23). In doing so, the coefficient functions of the $\mathcal{O}\left(\delta^{3}\right)$ remainders will contain up to three derivatives of the underlying wave profile $u_{0}$, and hence these functions will be $C^{r+2}$ functions of $\delta \in\left[0, \delta_{0}\right)$. One can now repeat the above argument by differentiating the fixed point relation (3.25) twice in order to verify that the functions $Y_{j}, j=1,2,3$, are $C^{2}$ functions of $\delta$ at $\delta=0$. As above, $C^{2}$ regularity in $\delta$ at $\delta=0$ of $w_{2}$ follows immediately. Finally, continuing this procedure inductively, one obtains $C^{r}$ regularity in $\delta \in\left[0, \delta_{0}\right)$ of the functions $Y_{j}, j=1,2,3$, and $w_{2}$.

Let us now consider $Y_{4}, w_{4}$ : with similar arguments, one shows that $\left(Y_{4}, w_{4}\right)$ are written as

$$
\left(Y_{4}, w_{4}\right)=\exp \left(\frac{X-w}{\delta}\right)\left(\delta^{2} \bar{Y}_{4},\left(1+\delta \bar{w}_{4}\right)\right),
$$

with $\left(\bar{Y}_{4}, \bar{w}_{4}\right)$ analytic with respect to $\lambda \in K$ and $C^{r}$ with respect to $\delta \in\left[0, \delta_{0}\right)$.

With the above preparations, we are now ready to expand the Evans function in $\delta$. At $\xi=0$, the resolvent matrix $\mathcal{R}$ of (3.24) reads

$$
\mathcal{R}(0, \lambda, \delta)=\left(\begin{array}{cc}
I d_{\mathbb{C}^{3}} & \exp \left(\int_{0}^{X} \mu(q) d q\right) \mathcal{O}\left(\delta^{2}\right) \\
0 & \exp \left(\int_{0}^{X} \mu(q) d q\right)(1+\mathcal{O}(\delta))
\end{array}\right),
$$

whereas at $\xi=X$, it reads

$$
\mathcal{R}(X, \lambda, \delta)=\left(\begin{array}{cc}
R(X, \lambda, \delta) & 0 \\
\mathcal{O}(\delta) & 1+\mathcal{O}(\delta)
\end{array}\right)
$$

where, with a slight abuse of notation, $\mathcal{O}\left(\delta^{m}\right)(m=1,2)$ are functions $f(\lambda, \delta)$ analytic in $\lambda \in K$ and $C^{r}$ functions of $\delta \in\left[0, \delta_{0}\right)$ that satisfy

$$
\lim _{\delta \rightarrow 0^{+}} \frac{\|f(\cdot, \delta)\|_{L^{\infty}(K)}}{\delta^{m}} \lesssim 1
$$

\footnotetext{
${ }^{13}$ Note that in the differentiation procedure, we lost one order of regularity of the profile with respect to $\delta$.
} 
Therefore, recalling that, by definition, $\Re(\mu(q)) \sim \delta^{-1}$ as $\delta \rightarrow 0$, it follows that

$$
\begin{aligned}
E(\lambda, \xi, \delta)= & \operatorname{det}\left(\mathcal{R}(X, \lambda, \delta)-e^{i \xi X} \mathcal{R}(0, \lambda, \delta)\right) \\
& =-(1+\mathcal{O}(\delta)) e^{i \xi X} \exp \left(\int_{0}^{X} \mu(q) d q\right) \bar{E}(\lambda, \xi, \delta),
\end{aligned}
$$

where

$$
\bar{E}(\lambda, \xi, \delta)=\operatorname{det}\left(R(X, \lambda, \delta)-e^{i \xi X} I d_{\mathbb{C}^{3}}\right)+\mathcal{O}\left(\delta^{3}\right)
$$

notice above we have expanded the Evans function with respect to the last column of the determinant to obtain the final equality. The function $\bar{E}(\lambda, \xi, \delta)$ is analytic with respect to $(\lambda, \xi)$ and $C^{r}$ with respect to $\delta \in\left[0, \delta_{0}\right)$. Recalling that $R(\cdot, \lambda, \delta)=$ $R_{\mathrm{KdV}}(\cdot, \lambda)+\delta R_{1}(\cdot, \lambda, \delta)$, the proposition follows.

3.3. Expansion of eigenvalues as $\delta \rightarrow 0$. By now considering the equation $\bar{E}(\lambda, \xi, \delta)=0$ for $0<\delta \ll 1$ and applying an appropriate implicit function argument, we deduce that if condition (A1) holds for a given $X \in \mathcal{W}$, then, for a given $X$-periodic traveling wave solution $u_{\delta}$, defined for all $\delta \in\left[0, \delta_{0}\right)$ for some $\delta_{0}$ sufficiently small, via Proposition 2.4, and for each fixed $\xi \in[-\pi / X, \pi / X)$ the eigenvalues of the Bloch operator $L_{\xi}\left[u_{\delta}\right]$ can be expanded in $\delta$ as $\delta \rightarrow 0$.

Corollary 3.8. Let $X \in \mathcal{W}$ and let $u_{\delta}$ be an $X$-periodic traveling wave solution of (1.1), defined for all $\delta \in\left[0, \delta_{0}\right)$ for some $\delta_{0}$ sufficiently small. Let $\xi \in[-\pi / X, \pi / X)$ be fixed and let $\lambda_{0}$ be a non-zero eigenvalue of $L_{\mathrm{KdV}, \xi}\left[u_{0}\right]$. If $X$ satisfies condition (A1), then there exists a $\delta_{1} \in\left(0, \delta_{0}\right)$ such that for all $\delta \in\left(0, \delta_{1}\right)$ there exists a unique eigenvalue $\lambda\left(\xi, \lambda_{0}, \delta\right)$ of $L_{\xi}\left[u_{\delta}\right]$ such that $\lambda\left(\xi, \lambda_{0}, \delta\right) \rightarrow \lambda_{0}$ as $\delta \rightarrow 0$ and, furthermore, for $0<\delta \ll 1$ this eigenvalue $\lambda\left(\xi, \lambda_{0}, \delta\right)$ can be expanded analytically in $\delta$ as

$$
\lambda\left(\xi, \lambda_{0}, \delta\right)=\lambda_{0}+\delta \lambda_{1}\left(\xi, \lambda_{0}\right)+\delta^{2} \lambda_{2}\left(\xi, \lambda_{0}\right)+\mathcal{O}\left(\delta^{3}\right)
$$

for some complex valued functions $\lambda_{1}, \lambda_{2}$.

Proof. The initial spectral problem $E(\lambda, \xi, \delta)=0$ is equivalent to $\bar{E}(\lambda, \xi, \delta)=0$ for $\delta$ sufficiently small. We proved in Proposition 3.7 that $\bar{E}$ is analytic with respect to $(\lambda, \xi) \in \mathbb{C}^{2}$ and, for any positive integer $r \geq 3$, can be chosen to be $C^{r}$ with respect to $\delta \in\left[0, \delta_{0}\right)$ for $\delta_{0}>0$ sufficiently small after choosing the profile $u_{\delta}$ to be sufficiently regular in $\delta$. Since condition (A1) is assumed to hold for $X \in \mathcal{W}$, the eigenvalue $\lambda_{0}$ is an isolated root of $\bar{E}(\cdot, \xi, 0)=E_{\mathrm{KdV}}(\cdot, \xi)$ so that one has $\partial_{\lambda} \bar{E}\left(\lambda_{0}(\xi), \xi\right) \neq 0$. The result follows by a combination of a Cauchy root counting argument and a straightforward application of the implicit function theorem.

Remark 3.9. Notice that the expansion of the eigenvalues provided by Corollary 3.8 is precisely the one that is assumed to exist in the work of Bar and Nepomnyashchy in $[\mathrm{BN}]$. Note, however, that for the moment this expansion is only expected to be uniformly valid for $|\xi| \geq \eta>0$, where $\eta$ is an arbitrarily small real number. In the following we do extend this expansion to the larger zone $0<\delta \ll \xi$. However, even this extended version fails to cover any neighborhood of the origin in the spectral plane. In Section 4, we will indeed see that the eigenvalues near $\lambda=0$ of the Bloch operator $L_{\xi}\left[u_{\delta}\right]$ do not expand smoothly in $\delta$ as $\delta \rightarrow 0$. 
From the above corollary, it follows that if conditions (A1)-(A3) are assumed to hold for a given $X \in \mathcal{W}$, then all the non-zero eigenvalues of the limiting $\mathrm{KdV}$ operator $L_{\mathrm{KdV}, 0}\left[u_{0}\right]$, with $u_{0}$ being an $X$-periodic traveling wave solution of the $\mathrm{KdV}$, bifurcate into the stable left half plane for $\delta$ sufficiently small. More precisely, we have the following result.

Corollary 3.10. Let $X \in \mathcal{P}$. Then for any $\eta_{1}>0$, there exist $\delta_{1}\left(X, \eta_{1}\right)>0$ and $\theta_{1}\left(X, \eta_{1}\right)>0$ such that for any $X$-periodic traveling wave solution $u_{\delta}$, defined for $\delta \in\left[0, \delta_{1}\right)$, we have that for all $\eta_{1} \leq|\xi| \leq \pi / X$

$$
\sigma\left(L_{\xi}\left[u_{\delta}\right]\right) \subset\left\{\lambda \mid \Re(\lambda) \leq-\theta_{1} \delta\right\}
$$

while for all $0 \leq|\xi| \leq \eta_{1}$ we have

$$
\sigma\left(L_{\xi}\left[u_{\delta}\right]\right) \cap B\left(0, \eta_{1}\right)^{c} \subset\left\{\lambda \mid \Re(\lambda) \leq-\theta_{1} \delta\right\} .
$$

Notice that the choices of $\delta_{1}$ and $\theta_{1}$ in Corollary 3.10 can be taken to be uniform when $X$ varies in a compact subset of $\mathcal{P}$. Corollary 3.10 effectively establishes the stability of the "high-frequnecy" part of the spectrum for any wave with $X \in \mathcal{P}$. Moreover, the results of this section rigorously justify the formal approach taken in [BN] to investigate necessary conditions, that is, $\operatorname{Ind}(X) \leq 0$, for stability of "nearKdV" solutions of the KdV-KS equation. Our goal is now to establish that the strict conditions, namely that $X \in \mathcal{P}$, is also sufficient for stability, thus completing the proof of Theorem 1.5. To this end, it remains to analyze how the spectrum organizes itself about the triple eigenvalue $\lambda=0$ of the limiting $\mathrm{KdV}$ operator $L_{\mathrm{KdV}, 0}\left[u_{0}\right]$ for sufficiently small $\delta$ and $\xi$. This is the goal of the next section.

\section{Spectrum at the origin and modulation equations}

As described above, at the present stage of the analysis, we already know that the formally derived and numerically evaluated criterion of $[\mathrm{BN}$ may be used to provide a first estimate of stability boundaries and that any undetected instability would necessarily be weak and correspond to long-wavelength perturbations. In this section, we complete the stability analysis initiated in the previous section by studying stability in the region $|(\lambda, \xi)| \ll 1$ using explicit calculations on a reduced Evans function designed to detect instabilities of $L_{\xi}\left[u_{\delta}\right]$ near the origin for $|(\lambda, \xi, \delta)| \ll 1$.

4.1. Spectral analysis through Evans function computations. We begin our study of the spectrum of the linearized operator $L_{\xi}$ in a neighborhood of the origin by analyzing the periodic Evans function $\bar{E}(\lambda, \xi, \delta)$, introduced in Proposition 3.7 . for $|(\lambda, \xi, \delta)|_{\mathbb{C} \times \mathbb{R} \times \mathbb{R}} \ll 1$. We begin with the following result.

Proposition 4.1. Let $X \in \mathcal{W}$ and consider the reduced Evans function $\bar{E}$ defined about an $X$-periodic traveling wave solution $u_{\delta}$, defined for $\delta \in\left[0, \delta_{0}\right)$ for some $\delta_{0}>0$ sufficiently small, of (1.1). In a sufficiently small neighborhood of $(\lambda, \xi, \delta)=$ $(0,0,0)$, then the spectral problem $\bar{E}(\lambda, \xi, \delta)=0$ is equivalen 14 to $\widetilde{E}(\lambda, \xi, \delta)=0$, with

$$
\widetilde{E}(\lambda, \xi, \delta)=\prod_{j=1}^{3}\left(\lambda-i \alpha_{j}(\xi) \xi\right)+\gamma \delta \prod_{k=1}^{2}\left(\lambda-i \beta_{k}^{0} \xi\right)+\delta^{2} \widetilde{E}_{2,2}(\lambda, \xi, \delta)+\delta \widetilde{E}_{3,1}(\lambda, \xi, \delta),
$$

\footnotetext{
${ }^{14}$ As in Proposition 3.7 by equivalence we mean that the functions are equal up to some non-vanishing multiplicative factor, which clearly preserves algebraic multiplicity of the zeros.
} 
where $\alpha_{j}(\xi) \in \mathbb{R}, j=1,2,3$, are as given in (1.3), the $\beta_{j} \in \mathbb{C}$ are either real or complex conjugates, and $\gamma \in \mathbb{R}$ is a constant. Given any positive integer $r \geq 1$, the functions $\widetilde{E}_{2,2}$ and $\widetilde{E}_{3,1}$ are analytic with respect to $(\lambda, \xi)$ in a sufficiently small neighborhood of the origin in $\mathbb{C} \times \mathbb{R}$, and is $C^{r}$ with respect to $\delta \in\left[0, \delta_{0}\right)$ for $\delta_{0}>0$ sufficiently small. Moreover, one has $\partial_{\lambda}^{l} \partial_{\xi}^{m} \widetilde{E}_{2,2}(0,0, \delta)=0$ if $l+m \leq 2$ and $\partial_{\lambda}^{l} \partial_{\xi}^{m} \widetilde{E}_{3,1}(0,0, \delta)=0$ if $l+m \leq 3$ for all $\delta \in\left[0, \delta_{0}\right)$.

Remark 4.2. When applying Proposition 4.1, we will discuss only loosely the constraint on the smallness of $\lambda$. Indeed, we already know from Proposition 3.7 that roots of $E$ in a given compact domain of the complex plane can be made uniformly close to roots of $E_{\mathrm{KdV}}$ by taking $\delta$ small; thus the amplitude of the former is essentially slaved to the size of $(\xi, \delta)$.

Proof. Recall from Proposition 3.7 that, after a suitable renormalization, in a sufficiently small neighborhood $\mathcal{N}_{1}$ of the origin $\lambda=0$ the Evans function expands as

$$
\bar{E}(\lambda, \xi, \delta)=E_{\mathrm{KdV}}(\lambda, \xi)+\delta E_{1}(\lambda, \xi)+\delta^{2} E_{2}(\lambda, \xi, \delta)
$$

for sufficiently small $\delta>0$. Now by using the fact that for all $\delta>0$ the dimension of the manifold of $\mathrm{KdV}-\mathrm{KS}$ periodic wave is 2 (indeed it is in one to one correspondence with a two dimensional submanifold of the $\mathrm{KdV}$ periodic waves if $\delta$ is small enough), one proves (see $[\mathrm{Se}$ for more details) that for all $\delta>0, \bar{E}(\lambda, \xi, \delta)$ expands, now with respect to $(\lambda, \xi) \approx(0,0)$, as

$$
\bar{E}(\lambda, \xi, \delta)=a(\delta) \lambda^{2}+b(\delta) \lambda \xi+c(\delta) \xi^{2}+P_{3}(\lambda, \xi, \delta)+E_{4}(\lambda, \xi, \delta),
$$

where, by choosing the profile $u_{\delta}$ to be sufficiently regular in $\delta$, and hence $\delta_{0}$ sufficiently small, $a, b, c:\left(0, \delta_{0}\right) \rightarrow \mathbb{C}$ are $C^{r}$ functions of $\delta$ and $P_{3}(\lambda, \xi, \delta)$ a homogeneous polynomial of degree 3 with respect to $(\lambda, \xi)$ for all $\delta$ and $C^{r}$ with respect to $\delta \in\left[0, \delta_{0}\right)$ ( $\delta_{0}$ small enough). The function $E_{4}$ is analytic with respect to $(\lambda, \xi) \in \mathcal{N}_{1} \times \mathcal{N}_{2}$, where $\mathcal{N}_{2} \subset \mathbb{R}$ is a sufficiently small neighborhood of $\xi=0$, and $C^{r}$ with respect to $\delta \in\left[0, \delta_{0}\right)$ so that $\partial_{\lambda}^{l} \partial_{\xi}^{m} E_{4}(0,0, \delta)=0$ for all $\delta>0$ and $l+m=3$. Furthermore, since for each fixed $\delta$ the function $\bar{E}(\lambda, \xi, \delta)$ is clearly real valued for $\lambda \in \mathbb{R}$ and $\xi \in \mathbb{R} i$, it follows that the functions $a$ and $c$ are real valued, while the function $b$ has range in $\mathbb{R} i$. Next, letting $\delta \rightarrow 0$, one finds

$$
a(0)=b(0)=c(0)=0, \quad E_{\mathrm{KdV}}(\lambda, \xi)=P_{3}(\lambda, \xi, 0)+E_{4}(\lambda, \xi, 0) .
$$

Hence, $\bar{E}$ now expands as

$$
\bar{E}(\lambda, \xi)=\delta\left(a_{0} \lambda^{2}+i b_{0} \lambda \xi+c_{0} \xi^{2}\right)+E_{\mathrm{KdV}}(\lambda, \xi)+\delta^{2} E_{2,2}(\lambda, \xi, \delta)+\delta E_{3,1}(\lambda, \xi, \delta),
$$

for some constants $a_{0}, b_{0}, c_{0} \in \mathbb{R} i$, where, for all $\delta \in\left[0, \delta_{0}\right), \partial_{\lambda}^{l} \partial_{\xi}^{m} E_{2,2}(0,0, \delta)=0$ for all $l+m \leq 2$ and $\partial_{\lambda}^{l} \partial_{\xi}^{m} E_{3,1}(0,0, \delta)=0$ for all $l+m \leq 3$.

Note that the principal part of $E_{\mathrm{KdV}}$ in its Taylor expansion with respect to $(\lambda, \xi)$ about $(0,0)$ is a homogeneous polynomial of degree 3 . Restricting to $|(\lambda, \xi)|_{\mathbb{C} \times \mathbb{R}} \ll$ 1 , the Weierstrass Preparation Theorem yields an expansion for $E_{\mathrm{KdV}}$ of the form

$$
E_{\mathrm{KdV}}(\lambda, \xi)=\Gamma(\lambda, \xi)\left(\lambda-i \alpha_{1}(\xi) \xi\right)\left(\lambda-i \alpha_{2}(\xi) \xi\right)\left(\lambda-i \alpha_{3}(\xi) \xi\right)
$$

where $\Gamma$ is a real valued analytic function defined in a sufficiently small neighborhood of the origin in $\mathbb{C} \times \mathbb{R}$ such that $\Gamma(0,0) \neq 0$ and the numbers $i \alpha_{j}(\xi) \xi$ are 
the roots of the associated Evans function $E_{\mathrm{KdV}}(\cdot, \xi)$ for the linearized $\operatorname{KdV}$ equation; see also (1.3). Then, the spectral problem $\bar{E}=0$ is equivalent, if $\lambda, \xi, \delta$ are sufficiently small, to $\widetilde{E}=0$ with

$$
\begin{aligned}
\widetilde{E}(\lambda, \xi, \delta)= & \delta \gamma\left(\lambda^{2}+i \tilde{b}_{0} \lambda \xi+\tilde{c}_{0} \xi^{2}\right)+\left(\lambda-i \alpha_{1}(\xi) \xi\right)\left(\lambda-i \alpha_{2}(\xi) \xi\right)\left(\lambda-i \alpha_{3}(\xi) \xi\right) \\
& +\delta^{2} \widetilde{E}_{2,2}(\lambda, \xi, \delta)+\delta \widetilde{E}_{3,1}(\lambda, \xi, \delta),
\end{aligned}
$$

for some constants $\tilde{b}_{0}, \tilde{c}_{0} \in \mathbb{R}$ and where $\widetilde{E}_{i, j}$ share the same properties as $E_{i, j}$ and $\gamma \in \mathbb{R}^{*}$.

Finally, notice that

$$
\lambda^{2}+i \tilde{b}_{0} \lambda \xi+\tilde{c}_{0} \xi^{2}=\left(\lambda-i \beta_{1}^{0} \xi\right)\left(\lambda-i \beta_{2}^{0} \xi\right),
$$

for some constants $\beta_{k}^{0}, k=1,2$, that are either real or complex conjugates. Inserting this into (4.1), we have proved that in a sufficiently small neighborhood of $(\lambda, \xi, \delta)=$ $(0,0,0)$, the spectral problem is equivalent, up to a non-vanishing function analytic in $(\lambda, \xi)$ and $C^{r}$ with respect to $\delta \in\left[0, \delta_{0}\right)$, to

$$
\widetilde{E}(\lambda, \xi, \delta)=\prod_{j=1}^{3}\left(\lambda-i \alpha_{j}(\xi) \xi\right)+\gamma \delta \prod_{k=1}^{2}\left(\lambda-i \beta_{k}^{0} \xi\right)+\delta^{2} \widetilde{E}_{2,2}(\lambda, \xi, \delta)+\delta \widetilde{E}_{3,1}(\lambda, \xi, \delta),
$$

with, for all $\delta>0, \partial_{\lambda}^{l} \partial_{\xi}^{m} \widetilde{E}_{2,2}(0,0, \delta)=0$ if $l+m \leq 2$ and $\partial_{\lambda}^{l} \partial_{\xi}^{m} \widetilde{E}_{3,1}(0,0, \delta)=0$ if $l+m \leq 3$. This concludes the proof of the proposition.

Remark 4.3. The real numbers $\alpha_{j}(0)$ and $\beta_{k}^{0}$ in the above proposition can be recognized as the characteristic speeds of the averaged Whitham modulation systems for the $\mathrm{KdV}$ and $\mathrm{KdV}-\mathrm{KS}$ equations, respectively, while the constant $\gamma$ is related to the relaxation dynamics associated with sending $\delta \rightarrow 0$ in the averaged Whitham modulation system associated with the KdV-KS equation 15 In the next section, we will explore this insightful connection in more detail. Besides, there we numerically evaluate these various numbers. We note, however, that the analysis in the current section is independent of any knowledge of this connection.

Given that Corollary 3.10 provides stability in Zone 1 of Figure 2, we restrict our attention now to a small neighborhood of $(\xi, \delta)=(0,0)$. We use the above asymptotic expansion of the periodic Evans function and split the neighborhood of the origin into three regions on which we apply different arguments; see Figure 2 for a pictorial description. Our first objective is to prove an extension of expansion (1.4):

$$
\lambda\left(\xi, \lambda_{0}, \delta\right) \stackrel{\delta / \xi \rightarrow 0}{=} \lambda_{0}+\delta \lambda_{1}\left(\xi, \lambda_{0}\right)+\mathcal{O}\left(\delta^{2}\right),
$$

uniform in both Zones 1 and 2, with $\lambda_{1}\left(\xi, \lambda_{0}\right)$ converging, as $\left(\xi, \lambda_{0}\right) \rightarrow(0,0)$, to one of three possible limits, depending on the spectral curve followed by $\left(\xi, \lambda_{0}\right)$. This shall extend stability up to Zone 2 provided the slope of the line boundary $C=|\xi| / \delta$ is large enough. In a second step, we extend this stability by a non-crossing argument to Zone 3, a disc sector $C^{-1}|\xi| \leq \delta \leq C|\xi|,|(\xi, \delta)| \leq \eta_{C}$, provided $\eta_{C}>0$ is small enough (depending on $C$ ). Since we already know that there is no crossing at the origin, we only need to exclude the crossing of non-zero eigenvalues of $L_{\xi}$ through the imaginary axis. Finally, we need to prove stability in the remaining

\footnotetext{
${ }^{15}$ In Section A.2 we provide an explicit formula for $\gamma$; see Remark A.1
} 
sector $|\xi| / \delta \leq C$, corresponding to Zone 4 in Figure 2, provided $C$ is large enough. To this purpose, we benefit from the fact that, in the process of handling Zone 1 , we prove that (A1)-(A3) imply a finite set of conditions, which coincide with the subcharacteristic conditions identified in NR2, and prove that these conditions yield a regular eigenvalue expansion as $(|\xi| / \delta, \delta) \rightarrow(0,0)$ implying that, up to choosing $\delta_{0}$ smaller, there exist $C>0$ and $\theta>0$ such that, for all $|\xi| \leq C \delta$, $\Re\left(\lambda\left(\xi, \lambda_{0}, \delta\right)\right) \leq-\theta \xi^{2} / \delta$ (whatever the choice of the corresponding $\lambda_{0}$ ). In carrying out this intricate three-regions proof, we elucidate the role of the subcharacteristic conditions, conjectured in NR2, by proving that they imply "low-frequency" stability while being implied by (A1)-(A3).

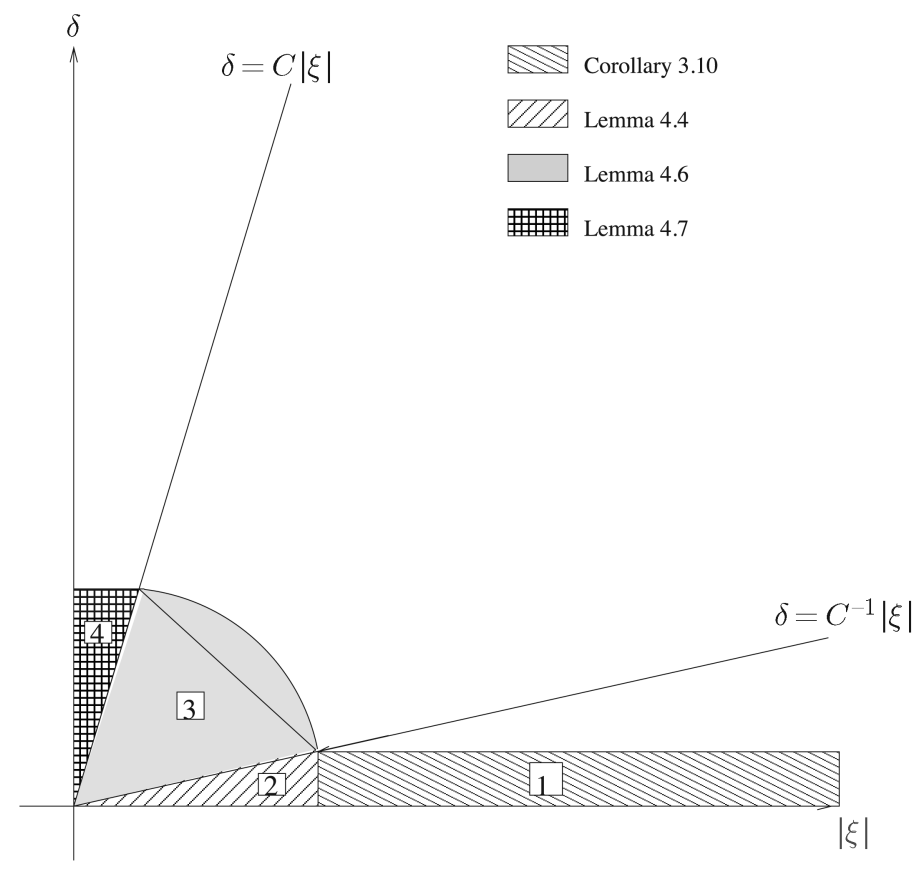

Figure 2. Domains in the $(|\xi|, \delta)$ plane where it is proved that there are no unstable eigenvalues. The middle sector is described by inequalities $C^{-1}|\xi| \leq \delta \leq C|\xi|,|(\xi, \delta)| \leq \eta_{C}$ with $C \gg 1$; then $\eta_{C} \ll 1$. Stability in Zone 1 corresponds to a rigorous validation of expansions as carried out in BN] (Corollary 3.10). Zone 2 is handled by providing an extension of the previous expansion (Lemma 4.4). The study of Zone 1 also provides that the subcharacteristic conditions (S1), (S2), (S3) are implied by (A1), (A2), (A3). In turn, the previous subcharacteristic conditions yield that in Zone 3 no eigenvalue can cross the imaginary axis, thus no unstable eigenvalue appears there (Lemma 4.6). At last, in Zone 4, a different kind of expansion is obtained from (S1), (S2), (S3) and proved to yield stability (Lemma 4.7). 
Let us start from the rescaled, reduced Evans function found in Proposition 4.1.

$$
\widetilde{E}(\lambda, \xi, \delta)=\prod_{j=1}^{3}\left(\lambda-i \alpha_{j}(\xi) \xi\right)+\gamma \delta \prod_{k=1}^{2}\left(\lambda-i \beta_{k}^{0} \xi\right)+\delta^{2} \widetilde{E}_{2,2}(\lambda, \xi, \delta)+\delta \widetilde{E}_{3,1}(\lambda, \xi, \delta),
$$

where $\gamma \in \mathbb{R}$ is constant, the $\beta_{k}^{0}$ are real or complex conjugate constants, and $\alpha_{j}(\xi) \in \mathbb{R}$. Notice that since the spectral curves for the linearized KdV equation obey the symmetry $\lambda(-\xi)=\bar{\lambda}(\xi)$, it follows that the $\alpha_{j}$ are even functions of $\xi$. Furthermore, letting $\xi \rightarrow 0$ in $\alpha_{j}(\xi)$ one obtains $\alpha_{j}(0)=\alpha_{j}^{0}, j=1,2,3$, where the $\alpha_{j}^{0}$ are the eigenvalues of the Whitham modulation system for the Kortewegde Vries equation; see [BrJ, BrJK, JZB, JZ1 for details. It follows that if the period $X \in \mathcal{W}$ of the underlying wave $u_{\delta}$ belongs to the set $\mathcal{P}$, i.e. if $X$ satisfies conditions (A1)-(A3), then the $\alpha_{j}^{0}$ are distinct and, without loss of generality, obey the ordering

$$
\alpha_{1}^{0}<\alpha_{2}^{0}<\alpha_{3}^{0} .
$$

We now carry out our first step that consists in extending (1.4). Actually we only need to do so for $0<\delta \leq C^{-1}|\xi|,|\xi|<\eta$, provided $\eta, C^{-1}>0$ are sufficiently small. This is the content of the following lemma.

Lemma 4.4. Let $X \in \mathcal{W}$ satisfy (A1) and (A2). Consider the Evans function $E$ defined about an $X$-periodic traveling wave solution of the $K d V$-KS equation (1.1). Then there exist constants $C_{0}, \eta_{0}>0$ and $M_{0}>0$ such that for all $0 \leq \delta \leq C_{0}^{-1}|\xi|$ and $|\xi| \leq \eta_{0}$, there are exactly three roots $\left\{\lambda_{k}(\xi, \delta)\right\}_{k=1,2,3}$ of the associated Evans function $E(\lambda, \xi, \delta)$ with $|\lambda| \leq M_{0}$. Moreover, these roots are smooth functions of $\xi \in\left(-\eta_{0}, \eta_{0}\right)$ and $\delta / \xi \in\left(-\varepsilon_{0}, \varepsilon_{0}\right)$ and expand, for $k=1,2,3$, as

$$
\begin{aligned}
& \lambda_{k}(\xi, \delta)=i \alpha_{k}(\xi) \xi-\gamma \delta \frac{\prod_{j=1}^{2}\left(\alpha_{k}(\xi)-\beta_{j}^{0}\right)}{\prod_{j \neq k}\left(\alpha_{k}(\xi)-\alpha_{j}(\xi)\right)}+\mathcal{O}(\delta \xi), \\
& \Re\left(\lambda_{k}(\xi, \delta)\right)=\delta A_{k}+\mathcal{O}(\delta \xi) \quad \text { with } \quad A_{k}=-\gamma \frac{\prod_{j=1}^{2}\left(\alpha_{k}^{0}-\beta_{j}^{0}\right)}{\prod_{j \neq k}\left(\alpha_{k}^{0}-\alpha_{j}^{0}\right)}
\end{aligned}
$$

when $|(\xi, \delta / \xi)| \rightarrow 0$. Moreover, one has $A_{k}<0$ for all $k=1,2,3$ if and only if the following conditions are satisfied:

(S1) $\beta_{1}^{0}, \beta_{2}^{0} \in \mathbb{R}$ and $\beta_{1}^{0} \neq \beta_{2}^{0}$;

(S2) $\alpha_{1}^{0}<\beta_{1}^{0}<\alpha_{2}^{0}<\beta_{2}^{0}<\alpha_{3}^{0}$ (once we have fixed $\beta_{1}^{0}<\beta_{2}^{0}$ );

(S3) $\gamma>0$.

In particular,

- if (S1)-(S3) hold, then there exist $\eta_{1}(X)>0, C_{0}(X)>0$ and $\theta_{0}(X)>0$ such that, provided $\delta_{0}$ is small enough, for a corresponding $X$-periodic wave $u_{\delta}, \delta \in\left[0, \delta_{0}\right)$, for all $\delta \leq C_{0}^{-1}|\xi|$,

$$
\sigma\left(L_{\xi}\left[u_{\delta}\right]\right) \cap B\left(0, \eta_{1}\right) \subset\left\{\lambda \mid \Re(\lambda) \leq-\theta_{0} \delta\right\} ;
$$

- condition (A3) implies conditions (S1)-(S3).

Remark 4.5. In what follows, the conditions (S1), (S2), and (S3) will be referred to as "the subcharacteristic conditions": this terminology will be justified in Section 4.2 below by discussing their formal derivation in NR2. There, we will recall the results of [NR2] that the $\beta_{j}^{0}$ are the limits, as $\delta$ goes to 0 , of the characteristics of the first order averaged Whitham modulation equations for (1.1). Hence, condition (S1) above simply states that the Whitham modulation system for (1.1), derived for 
fixed $\delta>0$, about the underlying wave is uniformly strictly hyperbolic in the limit $\delta \rightarrow 0$. Note that hyperbolicity of this system, corresponding to the requirement that $\beta_{j}^{0} \in \mathbb{R}$, is a well-known necessary condition for spectral stability to weak large-scale perturbations; see [Se, NR2]. We note furthermore that the condition (S1) is equivalent to the spectral assumption (H2) necessary to invoke the non-linear stability theory of [BJNRZ1.

Proof. Let $r \geq 3$ be a fixed positive integer and let $\delta_{0}>0$ be sufficiently small such that the underlying wave profile $u_{\delta}$ is at least a $C^{r}$ function of $\delta \in\left[0, \delta_{0}\right)$. As described above, for $(|\lambda|,|\xi|, \delta)$ sufficiently small the equation $E(\lambda, \xi, \delta)=0$ is equivalent to

$$
\prod_{j=1}^{3}\left(\lambda-i \alpha_{j}(\xi) \xi\right)+\gamma \delta \prod_{k=1}^{2}\left(\lambda-i \beta_{k}^{0} \xi\right)+\delta^{2} \widetilde{E}_{2,2}(\lambda, \xi, \delta)+\delta \widetilde{E}_{3,1}(\lambda, \xi, \delta)=0 .
$$

Now, setting $\delta=\bar{\delta} \xi, \lambda=\bar{\lambda} \xi$ and dividing (4.4) by $\xi^{3}$ yields the equation

$$
\widetilde{e}(\bar{\lambda}, \xi, \bar{\delta})=\prod_{j=1}^{3}\left(\bar{\lambda}-i \alpha_{j}(\xi)\right)+\gamma \bar{\delta} \prod_{k=1}^{2}\left(\bar{\lambda}-i \beta_{k}^{0}\right)+\xi \widetilde{e}_{2,2}(\bar{\lambda}, \xi, \bar{\delta})+\xi \widetilde{e}_{3,1}(\bar{\lambda}, \xi, \bar{\delta})=0
$$

with $\widetilde{e}_{2,2}=\xi^{-2} \bar{\delta}^{2} \widetilde{E}_{2,2}(\bar{\lambda} \xi, \xi, \bar{\delta} \xi)$ and $\widetilde{e}_{3,1}=\xi^{-3} \bar{\delta} \widetilde{E}_{3,1}(\bar{\lambda} \xi, \xi, \bar{\delta} \xi)$ analytic functions with respect to $\bar{\lambda}$ and $C^{r}$ with respect to $\xi$ and $\bar{\delta}$ near the origin. By comparing polynomial growth in $\bar{\lambda}$, it follows that there exist constants $\eta_{1}, M>0$, such that if $|\bar{\delta}|+|\xi|<\eta_{1}$, then $|\bar{\lambda}| \leq M<\infty$.

Now, letting $\bar{\delta}, \xi \rightarrow 0$ in 4.5 one finds that necessarily

$$
\widetilde{e}(\bar{\lambda}, 0,0)=\prod_{j=1}^{3}\left(\bar{\lambda}-i \alpha_{j}^{0}\right)=0 .
$$

Since $X$ satisfies condition $(\mathrm{A} 2)$, one has $\partial_{\bar{\lambda}} \widetilde{e}\left(i \alpha_{j}^{0}, 0,0\right) \neq 0$. Thus, the function $\widetilde{e}$ being analytic with respect to $\bar{\lambda}$ and $C^{r}$ with respect to $\bar{\delta}, \xi$, one shows, by applying to (4.5) a combination of a Cauchy counting argument and the implicit function theorem in a neighborhood of $\alpha_{j}^{0}$, that there exist, for $\xi, \bar{\delta}$ sufficiently small, exactly three roots $\left\{\bar{\lambda}_{j}(\xi, \bar{\delta})\right\}_{j=1,2,3}$ of (4.5) and that they are $C^{r}$ functions of $\xi$ and $\bar{\delta}$ and can be expanded as

$$
\bar{\lambda}_{j}(\xi, \bar{\delta})=i \alpha_{j}(\xi)-\gamma \bar{\delta} \frac{\left(\alpha_{j}(\xi)-\beta_{1}^{0}\right)\left(\alpha_{j}(\xi)-\beta_{2}^{0}\right)}{\prod_{k \neq j}\left(\alpha_{j}(\xi)-\alpha_{k}(\xi)\right)}+\mathcal{O}(\bar{\delta} \xi)
$$

Returning to the original variables via $\lambda_{j}(\xi, \delta)=\xi \bar{\lambda}_{j}(\xi, \bar{\delta})$, we obtain the desired regularity and expansions for the critical eigenvalues $\lambda_{j}, j=1,2,3$.

Recalling that the constants $\beta_{1}^{0}, \beta_{2}^{0}$ are either real or complex conjugate, we deduce by taking real parts

$$
\Re\left(\lambda_{j}(\xi, \delta)\right)=\delta A_{j}+\mathcal{O}\left(\delta \xi^{2}\right) \quad \text { with } \quad A_{j}=-\gamma \frac{\left(\alpha_{j}^{0}-\beta_{1}^{0}\right)\left(\alpha_{j}^{0}-\beta_{2}^{0}\right)}{\prod_{k \neq j}\left(\alpha_{j}^{0}-\alpha_{k}^{0}\right)} .
$$

By taking limits $\left(\xi, \lambda_{0}\right) \rightarrow 0$, along $\lambda_{0} \in \sigma\left(L_{\mathrm{KdV}, \xi}\right) \backslash\{0\}$, we also derive

$$
\max _{j=1,2,3} A_{j} \leq \operatorname{Ind}(X)
$$


hence proving that condition (A3) yields $\max _{j=1,2,3} A_{j}<0$. Since it follows from elementary computations that conditions (S1)-(S3) imply $\max _{j=1,2,3} A_{j}<0$, the only thing left is the proof of the converse.

Let us assume that $\max _{j=1,2,3} A_{j}<0$ and prove that the conditions (S1)-(S3) are satisfied. First, we suppose that $\beta_{1}^{0}, \beta_{2}^{0}$ are complex conjugates. In this case our assumption implies for any $j$ that $\gamma \neq 0$ and $\left(\alpha_{j}^{0}-\beta_{1}^{0}\right)\left(\alpha_{j}^{0}-\beta_{2}^{0}\right)=\left|\alpha_{j}^{0}-\beta_{1}^{0}\right|^{2}>0$, and hence

$$
\operatorname{sgn}\left(A_{j}\right)=-\operatorname{sgn}(\gamma) \operatorname{sgn}\left(\prod_{k \neq j}\left(\alpha_{j}^{0}-\alpha_{k}^{0}\right)\right) .
$$

Since $\alpha_{1}^{0}<\alpha_{2}^{0}<\alpha_{3}^{0}$, it follows that, contrary to our hypothesis, the $A_{j}$ cannot have all the same sign. Thus, it must be the case that the $\beta_{j}^{0}$ are real and distinct, verifying condition (S1). Taking without loss of generality $\beta_{1}^{0}<\beta_{2}^{0}$, it is now an easy computation to show that the signs of $\left(A_{j}\right)_{j=1,2,3}$ are the same if and only if the condition (S2) is satisfied. In this case, one has $\operatorname{sgn}\left(A_{j}\right)=-\operatorname{sgn}(\gamma)$ for $j=1,2,3$, hence condition (S3) must hold. This verifies that conditions (S1), (S2), and (S3) hold provided $\max _{j=1,2,3} A_{j}<0$ and completes the proof of the lemma.

To emphasize the crucial role of conditions (S1)-(S3) in determining the stability in a small neigborhood of the origin $(\xi, \delta, \lambda)=(0,0,0)$, we go on assuming the previous conditions instead of the stronger condition (A3). We now carry out our second step, proving that these conditions imply the absence of small imaginary eigenvalues in $C^{-1}|\xi| \leq \delta \leq C|\xi|,|(\xi, \delta)| \leq \eta_{C}$ provided $\eta_{C}$ is small enough.

Lemma 4.6. Let $X \in \mathcal{W}$ and assume that conditions (A1), (A2), (S1), (S2), and (S3) hold. Then for any $C>1$, there exists a constant $\eta_{C}>0$ such that, in a neighborhood of the origin, there is no crossing through the imaginary axis of any root of $E(\cdot, \xi, \delta)$ in the parameter region defined by $C^{-1}|\xi| \leq \delta \leq C|\xi|,|(\xi, \delta)| \leq \eta_{C}$. More precisely, for $(\xi, \delta)$ in this region,

- if $\xi=0$, then $\delta=0$ and the only root in a neighborhood of the origin is $\lambda=0$, which has algebraic multiplicity three;

- if $\xi \neq 0$, then, in some neighborhood of the origin, there is no imaginary root.

Proof. Let $C>1$ be given. We start by choosing $\eta_{C}$ small enough so that we may indeed replace $E$ with $\widetilde{E}$. The statement about the case $\xi=0$ is trivial, but, combined with a root counting argument, it implies a non-crossing through zero provided that $\eta_{C}>0$ is sufficiently small.

Let us now focus on the case $\xi \neq 0$. Suppose there exist $\left(\xi^{*}, \delta^{*}\right)$ with $\xi^{*} \neq 0$, $\left|\left(\xi^{*}, \delta^{*}\right)\right| \leq \eta_{C}$ and $\delta^{*} \in\left[C^{-1}\left|\xi^{*}\right|, C\left|\xi^{*}\right|\right]$ and a small real number $\tau^{*}$ such that $\widetilde{E}\left(i \tau^{*}, \xi^{*}, \delta^{*}\right)=0$. Then, taking real and imaginary parts in (4.2) yields the system of equations

$$
\begin{gathered}
\prod_{j=1}^{3}\left(\tau^{*}-\alpha_{j}\left(\xi^{*}\right) \xi^{*}\right)=\left(\delta^{*}\right)^{2} \Im\left(\widetilde{E}_{2,2}\left(i \tau^{*}, \xi^{*}, \delta^{*}\right)\right)+\delta^{*} \Im\left(\widetilde{E}_{3,1}\left(i \tau^{*}, \xi^{*}, \delta^{*}\right)\right), \\
\prod_{j=1}^{2}\left(\tau^{*}-\beta_{j}^{0} \xi^{*}\right)=\left(\delta^{*}\right)^{2} \Re\left(\widetilde{E}_{2,2}\left(i \tau^{*}, \xi^{*}, \delta^{*}\right)\right)+\delta^{*} \Re\left(\widetilde{E}_{3,1}\left(i \tau^{*}, \xi^{*}, \delta^{*}\right)\right) .
\end{gathered}
$$

Dividing the first of these equations by $\left(\xi^{*}\right)^{3}$ and using the facts $\partial_{\lambda}^{l} \partial_{\xi}^{m} \widetilde{E}_{2,2}(0,0, \delta)$ $=0$ for all $l+m \leq 2, \partial_{\lambda}^{l} \partial_{\xi} \widetilde{E}_{3,1}(0,0, \delta)=0$ for all $l+m \leq 3$, and $\frac{\delta^{*}}{\xi^{*}}$ is uniformly 
bounded in the region we are working in, it follows that given any $\varepsilon>0$, by possibly choosing $\eta_{C}>0$ smaller than above, there exists a $j \in\{1,2,3\}$ such that

$$
\left|\frac{\tau^{*}}{\xi^{*}}-\alpha_{j}^{0}\right|<\frac{\varepsilon}{2}
$$

Similarly, by dividing the second equation in (4.8) by $\left(\xi^{*}\right)^{2}$ it follows that for any $\varepsilon>0$, by possibly choosing $\eta_{C}>0$ above even smaller, there exists a $k \in\{1,2\}$ such that

$$
\left|\frac{\tau^{*}}{\xi^{*}}-\beta_{k}^{0}\right|<\frac{\varepsilon}{2} .
$$

From condition (S2), we know that we may apply the above arguments with

$$
\varepsilon=\min _{(j, k) \in\{1,2,3\} \times\{1,2\}}\left|\alpha_{j}^{0}-\beta_{k}^{0}\right|
$$

and thus obtain a contradiction. This achieves the proof of the lemma.

At last, under the same conditions as in the previous lemma, we investigate the region $|\xi| \leq C^{-1} \delta, 0<\delta \leq \delta_{0}$. Note that for fixed $\delta>0$, this is the region that contains small Floquet parameters $\xi$. Hence this is where we should read for a given "near-KdV" wave whether conditions (H1), (H2), (D2), (D3) are satisfied.

Lemma 4.7. Let $X \in \mathcal{W}$ satisfy conditions (A1), (A2), (S1), (S2) and (S3). Provided $C>0$ is large enough and $\delta_{0}>0$ is small enough, then, in some neighborhood of the origin, for any $(\xi, \delta)$ such that $0 \leq|\xi| \leq C^{-1} \delta$ and $0<\delta \leq \delta_{0}$, there are exactly three roots $\left\{\lambda_{j}(\xi, \delta)\right\}_{j=1,2,3}$ of the associated Evans function $E(\cdot, \xi, \delta)$. These roots are $\mathcal{C}^{r}$ functions of $(\delta, \xi / \delta)$ and expand as

$$
\begin{array}{lll}
\lambda_{1}(\xi, \delta)=i \beta_{1}^{0} \xi+B_{1} \frac{\xi^{2}}{\delta}+\mathcal{O}\left(\delta|\xi|+\frac{|\xi|^{3}}{\delta^{2}}\right), & \Re\left(\lambda_{1}(\xi, \delta)\right)=B_{1} \frac{\xi^{2}}{\delta}+\mathcal{O}\left(|\xi|^{2}+\frac{|\xi|^{3}}{\delta^{2}}\right), \\
\lambda_{2}(\xi, \delta)=i \beta_{2}^{0} \xi+B_{2} \frac{\xi^{2}}{\delta}+\mathcal{O}\left(\delta|\xi|+\frac{|\xi|^{3}}{\delta^{2}}\right), & \Re\left(\lambda_{2}(\xi, \delta)\right)=B_{2} \frac{\xi^{2}}{\delta}+\mathcal{O}\left(|\xi|^{2}+\frac{|\xi|^{3}}{\delta^{2}}\right), \\
\lambda_{3}(\xi, \delta)=B_{3} \delta+o(\delta), & \Re\left(\lambda_{3}(\xi, \delta)\right)=B_{3} \delta+o(\delta)
\end{array}
$$

as $|(\delta, \xi / \delta)| \rightarrow 0$, with

$$
B_{j}=\frac{\prod_{j=1}^{3}\left(\beta_{2}^{0}-\alpha_{j}^{0}\right)}{\gamma \prod_{k \neq j}\left(\beta_{j}^{0}-\beta_{k}^{0}\right)}, \quad j=1,2, \quad \text { and } \quad B_{3}=-\gamma .
$$

Moreover,

$$
\max _{j=1,2,3} B_{j}<0 .
$$

In particular, there exist $\eta_{1}(X)>0, C_{0}(X)>0$ and $\theta_{0}(X)>0$ such that, provided $\delta_{0}$ is small enough, for a corresponding $X$-periodic wave $u_{\delta}, \delta \in\left(0, \delta_{0}\right)$, conditions (H1), (H2) and (D3) hold, and, for all $|\xi| \leq C_{0}^{-1} \delta$,

$$
\sigma\left(L_{\xi}\left[u_{\delta}\right]\right) \cap B\left(0, \eta_{1}\right) \subset\left\{\lambda \mid \Re(\lambda) \leq-\theta_{0} \frac{|\xi|^{2}}{\delta}\right\} .
$$

Note that the expansions of the previous lemma preclude smooth eigenvalue expansions with respect to $(\xi, \delta)$ in a neighborhood of the origin.

Proof. Let $C>1$ be given. We choose $\delta_{0}$ small enough so that we may replace $E$ with $\widetilde{E}$. First, dividing (4.2) by $\delta^{3}$ and setting $\lambda=\bar{\lambda} \delta, \xi=\bar{\xi} \delta$ yields the equation

$$
\prod_{j=1}^{3}\left(\bar{\lambda}-i \alpha_{j}(\delta \bar{\xi}) \bar{\xi}\right)+\gamma \prod_{k=1}^{2}\left(\bar{\lambda}-i \beta_{k}^{0} \bar{\xi}\right)+\delta \widetilde{e}_{2,2}(\bar{\lambda}, \bar{\xi}, \delta)+\delta \widetilde{e}_{3,1}(\bar{\lambda}, \bar{\xi}, \bar{\delta})=0
$$


with $\widetilde{e}_{2,2}=\delta^{-2} \widetilde{E}_{2,2}(\bar{\lambda} \delta, \bar{\xi} \delta, \delta)$ and $\widetilde{e}_{3,1}=\delta^{-3} \widetilde{E}_{3,1}(\bar{\lambda} \delta, \bar{\xi} \delta, \delta)$ analytic with respect to $(\bar{\lambda}, \bar{\xi})$ and $C^{r}$ with respect to $\delta$. Then, for $|(\delta, \bar{\xi})|$ sufficiently small, one obtains by comparing polynomial growth a uniform bound on possible roots $\bar{\lambda}$. Now a root counting argument yields that there are exactly three roots, two $\bar{\lambda}_{1}, \bar{\lambda}_{2}$ lying in an $o(1)$-neighborhood of the origin and one $\bar{\lambda}_{3}$ contained in an $o(1)$-neighborhood of $-\gamma$, when $(\delta, \bar{\xi}) \rightarrow 0$. Undoing the renormalization already yields the expected expansion of $\lambda_{3}$ and that the two other roots belong to an $o(\delta)$-neighborhood of the origin when $(\delta, \bar{\xi}) \rightarrow 0$.

Let us choose a neighborhood of the origin small enough so that it contains only the two roots $\lambda_{1}$ and $\lambda_{2}$ and focus on this neighborhood. We expect, for $j=1,2$, not only the known $\lambda_{j}=o(\delta)$ but $\lambda_{j}=\mathcal{O}(\xi)$. In order to get this, we note that (4.9) implies

$$
\bar{\lambda}_{j}^{2}=\frac{\mathcal{O}\left(\left|\bar{\lambda}_{j}\right||\bar{\xi}|+|\bar{\xi}|^{2}\right)}{\bar{\lambda}_{j}+\gamma+\mathcal{O}(|\bar{\xi}|)}=\mathcal{O}\left(\left|\bar{\lambda}_{j}\right||\bar{\xi}|+|\bar{\xi}|^{2}\right), \quad j=1,2,
$$

so that a growth comparison provides $\bar{\lambda}_{j}^{2}=\mathcal{O}\left(|\bar{\xi}|^{2}\right), j=1,2$. To go further, we replace the previous eigenvalue scaling by $\lambda=\tilde{\lambda} \xi$, or equivalently $\bar{\lambda}=\tilde{\lambda} \bar{\xi}$, and rewrite (4.9) as

$$
\bar{\xi} \prod_{j=1}^{3}\left(\tilde{\lambda}-i \alpha_{j}(\delta \bar{\xi})\right)+\gamma \prod_{k=1}^{2}\left(\tilde{\lambda}-i \beta_{k}^{0}\right)+\delta \widetilde{\widetilde{e}}_{2,2}(\tilde{\lambda}, \bar{\xi}, \delta)+\delta \bar{\xi} \widetilde{\widetilde{e}}_{3,1}(\tilde{\lambda}, \bar{\xi}, \bar{\delta})=0
$$

with $\widetilde{\widetilde{e}}_{2,2}=(\delta \bar{\xi})^{-2} \widetilde{\widetilde{E}}_{2,2}(\tilde{\lambda} \bar{\xi} \delta, \bar{\xi} \delta, \delta)$ and $\widetilde{\widetilde{e}}_{3,1}=(\delta \bar{\xi})^{-3} \widetilde{E}_{3,1}(\tilde{\lambda} \bar{\xi} \delta, \bar{\xi} \delta, \delta)$. Then, for $j=1,2$, applying the implicit function theorem in a neighborhood of $(\tilde{\lambda}, \bar{\xi}, \delta)=$ $\left(i \beta_{j}, 0,0\right)$ yields the relevant regularity and the expected expansion

$$
\tilde{\lambda}_{j}(\bar{\xi}, \delta)=i \beta_{j}^{0}+B_{j} \bar{\xi}+\mathcal{O}\left(\delta+|\bar{\xi}|^{2}\right) .
$$

Then, for $j=1,2$, by using that $\Re \tilde{\lambda}_{j}$ is an odd function of $\bar{\xi}$, one obtains

$$
\Re\left(\tilde{\lambda}_{j}(\bar{\xi}, \delta)\right)=B_{j} \bar{\xi}+\mathcal{O}\left(\delta|\bar{\xi}|+|\bar{\xi}|^{2}\right) .
$$

Moreover, by an elementary investigation, the sign of the $B_{j}$ is deduced from (S1)(S3).

Condition (D3) and the conclusion on the spectrum $\sigma\left(L_{\xi}\left[u_{\delta}\right]\right)$ directly follow from the expansions. In turn it is known that (D3) implies (H1); see [NR2, Lemma 2.1]. At last, provided $\delta_{0}$ is small enough, condition (H2) follows from (S1) and, for $j=1,2, \beta_{j}=\beta_{j}^{0}+\mathcal{O}(\delta)$.

Proof of Theorem 1.5. By gathering the results of Corollary 3.10 and Lemmas 4.4. 4.7. 4.6 and 4.7. we achieve the proof of Theorem 1.5. Indeed, one may complete the explicit bounds on the real part of the spectrum contained in Corollary 3.10 and Lemmas 4.4 and 4.7 by noting that the non-crossing argument of Lemma 4.6 combined with a uniformity argument and a comparison on boundaries where one may apply either Lemma 4.4 or Lemma 4.7 yields that, provided $C_{0}$ is large enough and $\delta_{0}$ is sufficiently small, for a corresponding $X$-periodic wave $u_{\delta}, \delta \in\left(0, \delta_{0}\right)$, there exists $\theta_{0}(X, \delta)>0$ such that, for all $C_{0}^{-1} \delta \leq|\xi| \leq C_{0} \delta$,

$$
\sigma\left(L_{\xi}\left[u_{\delta}\right]\right) \cap B\left(0, \eta_{1}\right) \subset\left\{\lambda \mid \Re(\lambda) \leq-\theta_{0}\right\} .
$$

Hence (D3) follows from Corollary 3.10 for large $\lambda$, and, for small $\lambda$, from Lemmas 4.7. 4.7 and 4.4, and Corollary 3.10 respectively for $|\xi| \leq C_{0}^{-1} \delta, C_{0}^{-1} \delta \leq|\xi| \leq C_{0} \delta$, 
$C_{0} \delta \leq|\xi| \leq \eta_{1}$ and $\eta_{1} \leq|\xi| \leq \pi / X$. Uniformity of $\delta_{0}(\cdot)$ is deduced from a close inspection of the proof.

Before we continue, we emphasize that it follows from Lemmas 4.4, 4.6 and 4.7 that, when (A1) and (A2) hold, the subcharacteristic conditions (S1), (S2), and (S3) are sufficient for the diffusive spectral stability under slow low-Floquet perturbations 16 Given this role and though we know from Lemma 4.4 that they are implied by (A1)-(A3), we wish to provide an independent numerical verification of the subcharacteristic conditions (S1)-(S3) that are independent of the numerical calculations in $[\mathrm{BN}]$ or, equivalently, independent of the numerical investigation of condition (A3). In the process, we will justify our terminology in referring to (S1)-(S3) as the subcharacteristic conditions. The terminology comes directly from the singular version of the Whitham modulation theory developed in [NR2]. Actually, this is precisely on the basis of the following formal discussion that the subcharacteristic conditions (S1)-(S3) were first conjectured to play a major in the slow/small-Floquet stability of "near-KdV" waves [NR2].

4.2. Whitham's modulation equations. It is now a classical result that Whitham's modulation equations for periodic waves of conservation laws provide an accurate description of the spectral curves at the origin, i.e. of the stability of a given wave train to weak large-scale perturbations Let us mention here the work [Se] in the general case, [NR1] for shallow water equations, NR2] for KdV-KS either for fixed $\delta>0$ or in the KdV limit and [JZB, JZ1 for the generalized Kortewegde Vries equation. When considering (1.1) in the singular limit $\delta \rightarrow 0^{+}$, however, even the formal derivation of such a connection is more involved. In particular, we note that it is not sufficient to simply let $\delta \rightarrow 0$ in the modulation equations derived for (1.1) with $\delta>0$ fixed. Instead, in this singular limit the introduction of a new set of modulation equations is required [NR2]. In this section, we recall the derivation of the appropriate modulation equations in this singular limit and emphasize in which way the previous analysis demonstrates their connection with the spectrum at the origin of the linearized operator about a given wave train. In particular, the structure of the modulation equations will justify our terminology, referring to conditions (S1), (S2), and (S3) as the "subcharacteristic" conditions.

Recall that the KdV-KS equation reads

$$
\partial_{t} u+\partial_{x}\left(\frac{u^{2}}{2}\right)+\partial_{x}^{3} u+\delta\left(\partial_{x}^{2} u+\partial_{x}^{3} u\right)=0 .
$$

Reproducing [NR2], we derive the Whitham modulation equations about a given periodic wave train of (4.11) in the singular limit $\delta \rightarrow 0^{+}$. To this end, we introduce the slow coordinates $(X, T)=(\varepsilon x, \varepsilon t), \varepsilon \ll 1$, set $\delta=\bar{\delta} \varepsilon$ with $\bar{\delta} \in(0, \infty)$, and note that in the slow $(X, T)$ variables equation (4.11) reads

$$
\partial_{T} u+\partial_{X}\left(\frac{u^{2}}{2}\right)+\varepsilon^{2} \partial_{X}^{3} u+\bar{\delta}\left(\varepsilon^{2} \partial_{X}^{2} u+\varepsilon^{4} \partial_{X}^{4} u\right)=0 .
$$

Following [Se, we search for an expansion of $u$, a solution of (4.12), in the form

$$
u(X, T)=U^{(0)}\left(\frac{\phi(X, T)}{\varepsilon} ; X, T\right)+\varepsilon U^{(1)}\left(\frac{\phi(X, T)}{\varepsilon}, X, T\right)+\mathcal{O}\left(\varepsilon^{2}\right)
$$

\footnotetext{
${ }^{16}$ That is corresponding to $|\lambda| \leq \eta_{1}$ and $|\xi| \leq \eta_{1}$, with $\eta_{1}$ sufficiently small independently of $\delta$.
} 
with $U^{(i)}(y ; X, T)$ 1-periodic in $y$. Notice then that the local period of oscillation of $u^{0}$ in the variable $y$ is $\varepsilon / \partial_{X} \phi$, where we assume the unknown phase a priori satisfies the condition $\partial_{X} \phi \neq 0$. By inserting this ansatz into (4.12) and collecting $\mathcal{O}\left(\varepsilon^{-1}\right)$ terms, one finds

$$
\Omega \partial_{y} U^{(0)}+\kappa U^{(0)} \partial_{y} U^{(0)}+\kappa^{3} \partial_{y}^{3} U^{(0)}=0,
$$

where $\Omega=\partial_{T} \phi$ and $\kappa=\partial_{X} \phi$. Equation (4.14) is recognized as the traveling wave ODE for the $\mathrm{KdV}$ equation (2.1) in the variable $\kappa y$ with wave speed $-\Omega / \kappa$. As such, equation (4.14) has a solution provided $\Omega=-\kappa c_{0}\left(u_{0}, \kappa, k\right)$, where now $u_{0}, \kappa$ and $k$ are considered as functions of the slow variables $(X, T)$. In this case, the solutions of (4.14) can be expressed as

$$
\begin{aligned}
& U^{(0)}(y, X, T)=U_{0}\left(y, u_{0}, \kappa, k\right)=u_{0}+12 k^{2} \kappa^{2} \mathrm{cn}^{2}(\kappa y, k), \\
& c_{0}\left(u_{0}, \kappa, k\right)=u_{0}+8 \kappa^{2} k^{2}-4 \kappa^{2} .
\end{aligned}
$$

In what follows, we derive a system of "modulation equations" describing the evolution of $\left(u_{0}, \kappa, k\right)$ as functions of the slow variables $(X, T)$. One such equation comes from noticing that the compatibility condition $\partial_{T} \kappa=\partial_{X} \Omega$ yields the equation

$$
\partial_{T} \kappa+\partial_{X}\left(\kappa c_{0}\left(u_{0}, \kappa, k\right)\right)=0
$$

for the local wave number $\kappa$.

To find other modulation equations we continue the above expansion and note that collecting the $\mathcal{O}(1)$ terms yields an equation of the form $L_{\mathrm{KdV}, 0} U^{(1)}=\ldots$, where $L_{\mathrm{KdV}, 0}$ is the operator describing the linearized evolution of the $\mathrm{KdV}$ equation about $U^{(0)}$. Since the kernel of the adjoint of $L_{\mathrm{KdV}, 0}$ is spanned by 1 and $U^{(0)}$, solvability conditions and thus the needed extra equations will be obtained by averaging in $y$ against 1 and $U^{(0)}$ the $\mathcal{O}(1)$ equation. Yet with this equation being of the form

$$
\partial_{T} U^{(0)}+\partial_{X}\left(\frac{\left(U^{(0)}\right)^{2}}{2}\right)=\partial_{y}(\cdots)
$$

it follows, averaging it over a single period in $y$, that

$$
\partial_{T}\left\langle U_{0}\left(\cdot ; u_{0}, \kappa, k\right)\right\rangle+\partial_{X}\left\langle\frac{U_{0}^{2}}{2}\left(\cdot, u_{0}, \kappa, k\right)\right\rangle=0
$$

must be satisfied, where here $\langle f\rangle:=\int_{0}^{1} f(y) d y$. To obtain the other solvability condition in an easy way, let us first remark, following the method used in [JZ1] to derive modulations equations for the generalized Korteweg-de Vries equation, that by multiplying (4.11) by $u$ we obtain an equation of the form

$$
\partial_{t}\left(\frac{u^{2}}{2}\right)+\partial_{x}\left(\frac{u^{3}}{3}-\frac{3\left(\partial_{x} u\right)^{2}}{2}\right)=\delta\left(\left(\partial_{x} u\right)^{2}-\left(\partial_{x}^{2} u\right)^{2}\right)+\partial_{x}^{2}(\cdots) .
$$

This implies that equation (4.17) multiplied by $U_{0}$ yields

$$
\partial_{T}\left(\frac{U_{0}^{2}}{2}\right)+\partial_{X}\left(\frac{U_{0}^{3}}{3}-\frac{3\left(U_{0}^{\prime}\right)^{2}}{2}\right)=\bar{\delta}\left(\left(U_{0}^{\prime}\right)^{2}-\left(U_{0}^{\prime \prime}\right)^{2}\right)+\partial_{y}(\cdots) .
$$

Averaging (4.20) over a period in $y$ then provides the balance law

$$
\partial_{T}\left\langle\frac{U_{0}^{2}}{2}\right\rangle+\partial_{X}\left\langle\frac{U_{0}^{3}}{3}-\frac{3\left(U_{0}^{\prime}\right)^{2}}{2}\right\rangle=\bar{\delta}\left(\left\langle\left(U_{0}^{\prime}\right)^{2}\right\rangle-\left\langle\left(U_{0}^{\prime \prime}\right)^{2}\right\rangle\right) .
$$


Together, the homogenized system (4.16), (4.18), (4.21) forms a closed system of three conservation laws with a source term, called the averaged Whitham modulation system, describing the evolution of the quantities $\left(u_{0}, \kappa, k\right)$ as functions of the slow variables $(X, T)$.

Again repeating [NR2, let us now comment on the previous system. As a first step in analyzing the modulation system (4.16), (4.18), (4.21), notice that the steady states are given by points $\left(u_{0}^{\star}, \kappa^{\star}, k^{\star}\right) \in \mathbb{R}^{3}$ such that

$$
\left\langle\left(U_{0}^{\prime}\right)^{2}\left(\cdot, u_{0}^{\star}, \kappa^{\star}, k^{\star}\right)\right\rangle=\left\langle\left(U_{0}^{\prime \prime}\right)^{2}\left(\cdot, u_{0}^{\star}, \kappa^{\star}, k^{\star}\right)\right\rangle,
$$

where $U_{0}$ is given as in (4.15), i.e. $U_{0}$ corresponds to periodic traveling waves of (4.11) in the limit $\delta \rightarrow 0$. Indeed, by Proposition 2.4, these are simply the cnoidal wave trains of the KdV equation that can be continued as solutions of (1.1). Now, letting $\bar{\delta} \rightarrow 0$, corresponding to large scale perturbations with frequency/wave number of order $\varepsilon \gg \delta$, in the homogenized system (4.16), (4.18), (4.21) yields the Whitham averaged system for the Korteweg-de Vries equation; see [W, JZ1. As stated previously, the numerical results in Figure 3 below demonstrate that for all $\mathrm{KdV}$ cnoidal wave trains considered here the Whitham averaged system for (2.1) is strictly hyperbolic with eigenvalues

$$
\alpha_{1}\left(u_{0}, \kappa, k\right)<\alpha_{2}\left(u_{0}, \kappa, k\right)<\alpha_{3}\left(u_{0}, \kappa, k\right), \quad \forall\left(u_{0}, \kappa, k\right) \in \mathbb{R}^{3} .
$$

Furthermore, in the limit $\bar{\delta} \rightarrow \infty$, corresponding to a relaxation limit and large scale perturbations with frequency/wave number $\varepsilon \ll \delta$, we obtain the relaxed system

$$
\begin{aligned}
& \partial_{T} \mathcal{G}(k)+\partial_{X}\left(\mathcal{G}(k) c_{0}\left(u_{0}, \mathcal{G}(k), k\right)\right)=0, \\
& \partial_{T}\left\langle U_{0}\left(\cdot ; u_{0}, \mathcal{G}(k), k\right)\right\rangle+\partial_{X}\left\langle\frac{U_{0}^{2}}{2}\left(\cdot ; u_{0}, \mathcal{G}(k), k\right)\right\rangle=0,
\end{aligned}
$$

where here $\kappa=\mathcal{G}(k)$ is given by the selection principal in Proposition 2.4, Notice that this system may also be obtained directly from the Whitham averaged system of conservation laws for the KdV-KS equation (1.1), derived in [NR2 for fixed $\delta>0$ as

$$
\partial_{T} \kappa+\partial_{X}\left(\kappa c_{\delta}(M, \kappa)\right)=0, \quad \partial_{T} M+\partial_{X}\left\langle\frac{U_{\delta}^{2}}{2}(M, \kappa)\right\rangle=0, \quad M=\left\langle U_{\delta}(M, \kappa)\right\rangle,
$$

in the limit as $\delta \rightarrow 0$. It is now well established [Se,NR2] that a necessary condition for spectral stability of periodic traveling waves under large scale perturbations is that system (4.22) be hyperbolic, i.e. have only real eigenvalues. In our analysis from Section 4.1, however, we assume the stronger condition that the modulation system (4.22) is strictly hyperbolic with eigenvalues

$$
\beta_{1}\left(u_{0}, k\right)<\beta_{2}\left(u_{0}, k\right) ;
$$

this corresponds precisely to condition $(S 1)$ in Lemma 4.4. It clearly follows that in considering only the relaxed hyperbolic system (4.22), obtained by simply letting $\delta \rightarrow 0$ in the Whitham modulation equations for (1.1) derived for fixed $\delta>0$, that some information is lost; namely, in this particular limit we obtain no information regarding conditions (S2) and (S3).

To understand the roles of conditions (S2) and (S3), we must consider rather the full modulation system (4.16), (4.18), (4.21) derived in the singular limit $\delta \rightarrow 0$. For the sake of clarity, let us write this system with the parameterization $(\kappa, M, E)$ with $M=\langle U\rangle$ corresponding to the spatial average of $U$ over a period and $E=\left\langle U^{2} / 2\right\rangle$; 
see [JZB, JZ1 for a discussion on such a parameterization of periodic wave trains of the KdV equation (2.1).

In this parameterization, the modulation system (4.16), (4.18), (4.21) recovers the form obtained in NR2,

$\partial_{T} \kappa-\partial_{X}(\Omega(\kappa, M, E))=0, \partial_{T} M+\partial_{X} E=0, \partial_{T} E+\partial_{X} Q(\kappa, M, E)=\bar{\delta} R(\kappa, M, E)$, where $\Omega(\kappa, M, E)=-\kappa c_{0}(\kappa, M, E), Q=\left\langle U_{0}^{3}-3\left(U_{0}^{\prime}\right)^{2} / 2\right\rangle$ and $R=\left\langle\left(U_{0}^{\prime}\right)^{2}-\left(U_{0}^{\prime \prime}\right)^{2}\right\rangle$. In the context of relaxation theory it is a classical assumption to suppose that the condition $\partial_{E} R\left(\kappa^{\star}, M^{\star}, E^{\star}\right) \neq 0$ is satisfied, ensuring that near the equilibrium state $\left(\kappa^{\star}, M^{\star}, E^{\star}\right)$ the equation $E(\kappa, M, E)=0$ defines $E$ implicitly in terms of $(\kappa, M)$; in what follows, we assume that this condition holds.

Under this assumption, the subcharacteristic condition (S3) can be easily interpreted. Indeed, linearizing the modulation system (4.24) about the steady state $\left(\kappa^{\star}, M^{\star}, E^{\star}\right)$ and restricting to spatially homogeneous, i.e. $X$-independent, perturbations yields the equation

$$
\partial_{T} \tilde{\kappa}=0, \quad \partial_{T} \tilde{M}=0, \quad \partial_{T} \tilde{E}=\bar{\delta}\left(\partial_{E} R^{\star} \tilde{E}+d_{\kappa, M} R^{\star}(\tilde{\kappa}, \tilde{M})\right),
$$

where $R^{\star}=R\left(\kappa^{\star}, M^{\star}, E^{\star}\right)$. Considered as a constant coefficient equation in the slow variables $(X, T)$, the dispersion relation of (4.25) is then given by

$$
\lambda^{2}\left(\lambda-\bar{\delta} \partial_{E} R^{\star}\right)=0 .
$$

From this, it is clear from our spectral analysis in Section4.1 that the condition (S3) is equivalent to $\partial_{E} R^{\star}<0$. We note that this condition is a standard assumption in the context of relaxation theory and is equivalent to requiring that the manifold of solutions of $R(\kappa, M, E)=0$ is stable.

Furthermore, the dispersion relation (4.26) implies that two spectral curves bifurcate from the origin as one allows the period of the perturbations to vary, corresponding to stability or instability with respect to weak long-wavelength perturbations. It is a classical result [W, $\mathrm{W}$ ] that a necessary condition for the stability of the steady states of (4.24) to such large-scale perturbations is given by the subcharacteristic condition

$$
\alpha_{1}^{\star} \leq \beta_{1}^{\star} \leq \alpha_{2}^{\star} \leq \beta_{2}^{\star} \leq \alpha_{3}^{\star},
$$

where here the $\alpha_{j}^{\star}$ and $\beta_{j}^{\star}$ denote the functions $\alpha_{j}^{0}$ and $\beta_{j}^{0}$, respectively, evaluated at the associated steady state. Notice that in our analysis from Section 4.1, however, we assume the stronger condition that the inequalities in (4.27) are strict, corresponding precisely to condition (S2).

In summary, we have just reviewed how conditions (S1), (S2), and (S3) were introduced in [NR2] as the strict subcharacteristic conditions for the relaxationtype Whitham modulation system (4.24), derived from (1.1) in the singular limit $\delta \rightarrow 0$. As a byproduct of the analysis, carried out in Section 4.1, of the exact role of conditions (S1)-(S3), our present work has also rigorously validated the role of the modulation system (4.24) in the determination of the presence of unstable spectrum near the origin for "near $\mathrm{KdV}$ " waves.

4.3. Numerical computation of subcharacteristic conditions. To finish this section, we use the explicit connection to the singular Whitham modulation system of [NR2], reviewed in Section 4.2, to provide an independent verification of the conditions $(\mathrm{S} 1)-(\mathrm{S} 3)$. In particular, we also rely on the parametrization of the 
"near-KdV" wave trains of (1.1) described in Proposition 2.4. We recall, however, that, when (A1) and (A2) hold, these subcharacteristic conditions follow directly from condition (A3), which was numerically investigated in [BN], and that, without (A3), we may only conclude to slow/small-Floquet spectral stability, which is insufficient to apply the recent analysis in BJNRZ1 that proves that any wave that satisfies (H1)-(H2) and (D1)-(D3) is non-linearly stable (in a suitable sense).

It is well known that the Whitham modulation equations for the KdV equation (2.1) can be diagonalized by quantities referred to as Riemann invariants; see [W]. To describe this diagonalization and introduce the appropriate set of Riemann invariants, we first recall some properties concerning the parametrization of the $\mathrm{KdV}$ wave trains. To begin, notice that traveling wave solutions of (2.1) are solutions of the form $u(x, t)=u(x-c t)$ for some $c \in \mathbb{R}$, where the profile $u(\cdot)$ satisfies the equation

$$
u u^{\prime}-c u^{\prime}+u^{\prime \prime \prime}=0 .
$$

Integrating once, one finds that the profile $u$ satisfies the Hamiltonian ODE

$$
u^{\prime \prime}+\frac{u^{2}}{2}-c u=a,
$$

for some constant of integration $a \in \mathbb{R}$, which can then be reduced to the form of a non-linear oscillator as

$$
\frac{\left(u^{\prime}\right)^{2}}{2}=q-W(u ; a, c), \quad W(u ; a, c)=\frac{u^{3}}{6}-c \frac{u^{2}}{2}-a u,
$$

where again $q$ denotes a constant of integration and $W$ represents the effective potential energy of the Hamiltonian ODE (4.28). On open sets of the parameter space $(a, q, c) \in \mathbb{R}^{3}$ the cubic polynomial $q-W(u ; a, c)$ has a positive discriminant so that there exist real numbers $u_{1} \leq u_{2} \leq u_{3}$ such that

$$
q-W(u ; a, c)=\frac{1}{6}\left(u-u_{1}\right)\left(u-u_{2}\right)\left(u_{3}-u\right) .
$$

By elementary phase plane analysis, it follows that for such $(a, q, c)$ the profile ODE (4.28) admits non-constant periodic solutions. Moreover, by identifying powers of $u$ we find in this parameterization that

$$
c=\frac{u_{1}+u_{2}+u_{3}}{3}, \quad a=-\frac{1}{6}\left(u_{1} u_{2}+u_{1} u_{3}+u_{2} u_{3}\right), \quad q=\frac{u_{1} u_{2} u_{3}}{6} .
$$

Using straightforward elliptic integral calculations, we find that the periodic solutions of (4.28) can be written in terms of the Jacobi cnoidal function $\operatorname{cn}(x, k)$ as

$$
u(\xi)=u_{2}+\left(u_{3}-u_{2}\right) \operatorname{cn}^{2}\left(\sqrt{\frac{u_{3}-u_{1}}{3}} \xi, k\right), \quad \xi=x-c t, \quad k^{2}=\frac{u_{3}-u_{2}}{u_{3}-u_{1}}
$$

In particular, notice that all solutions of (2.1) are of form (4.30) up to a Galilean shift and spatial translation. Letting $X=\frac{2 \pi}{\kappa}$ denote the period of the above wave train, it follows again by standard elliptic function considerations that $\kappa$ can be expressed as

$$
\kappa=\frac{\pi}{K(k)} \sqrt{\frac{u_{3}-u_{1}}{3}}
$$


where here

$$
K(k)=\int_{0}^{1} \frac{d x}{\sqrt{1-x^{2}} \sqrt{1-k^{2} x^{2}}}
$$

denotes the complete elliptic integral of the first kind.

Furthermore, in terms of this parameterization we note that

$$
\langle u\rangle=u_{1}+2\left(u_{3}-u_{1}\right) \frac{E(k)}{K(k)}, \quad\left\langle\frac{u^{2}}{2}\right\rangle=c\langle u\rangle+a,
$$

where here $\langle\cdot\rangle$ denotes the spatial average (in $\xi$ ) over a period $X$ and

$$
E(k)=\int_{0}^{1} \frac{\sqrt{1-k^{2} x^{2}}}{\sqrt{1-x^{2}}} d x
$$

denotes the complete elliptic integral of the second kind.

With this preparation, we can introduce the Riemann invariants $\left(\omega_{1}, \omega_{2}, \omega_{3}\right)$ for the KdV equation (2.1), which are defined in terms of the $u_{i}$ as

$$
\omega_{1}=\frac{u_{1}+u_{2}}{2}, \quad \omega_{2}=\frac{u_{1}+u_{3}}{2}, \quad \omega_{3}=\frac{u_{2}+u_{3}}{2} .
$$

In terms of this parameterization, we have

$$
\begin{aligned}
& u(\xi)=\omega_{1}+\beta_{3}-\omega_{2}+2\left(\omega_{2}-\omega_{1}\right) \mathrm{cn}^{2}\left(\sqrt{\frac{2\left(\omega_{3}-\omega_{1}\right)}{3}} \xi, k\right), \quad \kappa=\frac{2 \pi}{K(k)} \sqrt{\frac{2\left(\omega_{3}-\omega_{1}\right)}{3}}, \\
& c=\frac{\omega_{1}+\omega_{2}+\omega_{3}}{3}, \quad k^{2}=\frac{\omega_{2}-\omega_{1}}{\omega_{3}-\omega_{1}}, \quad\langle u\rangle=\omega_{1}+\omega_{2}-\omega_{3}+4\left(\omega_{3}-\omega_{1}\right) \frac{E(k)}{K(k)} \\
& \left\langle\frac{u^{2}}{2}\right\rangle=c\langle u\rangle+a, \quad a=-\frac{1}{6}\left(2 \beta_{1}\left(\omega_{2}+\omega_{3}-\omega_{1}\right)+\left(\omega_{1}+\omega_{2}-\omega_{3}\right)\left(\omega_{1}+\omega_{3}-\omega_{2}\right)\right) .
\end{aligned}
$$

The Whitham modulation equations for the KdV equations can be diagonalized by the Riemann invariants $\omega_{i}$, in the sense that they can be written as

$$
\partial_{T} \omega_{i}+V_{i}\left(\omega_{1}, \omega_{2}, \omega_{3}\right) \partial_{X} \omega_{i}=0
$$

where the characteristic velocities $V_{i}$ are given explicitly by

$$
V_{i}\left(\omega_{1}, \omega_{2}, \omega_{3}\right)=\frac{\partial_{\omega_{i}}(\kappa c)}{\partial_{\omega_{i}} \kappa}
$$

or, alternatively, as $V_{i}\left(\omega_{1}, \omega_{2}, \omega_{3}\right)=c+\left(\partial_{\omega_{i}} \ln (\kappa)\right)$. Clearly, the characteristic velocities $V_{i}\left(\omega_{1}, \omega_{2}, \omega_{3}\right)$ correspond to the eigenvalues of the Whitham modulation equations for (2.1) about the periodic traveling wave given in (4.30) associated with $\left(\omega_{1}, \omega_{2}, \omega_{3}\right)$. To describe these velocities more explicitly, we find it more convenient to parameterize the problem by the variables $\omega_{1}, \Delta=\omega_{3}-\omega_{1}$ and $k^{2}=$ $\left(\omega_{2}-\omega_{1}\right)\left(\omega_{3}-\omega_{1}\right)^{-1}$. In terms of $\left(\omega_{1}, \Delta, k^{2}\right)$, an elementary calculation shows that the characteristic velocities $V_{i}$ can be expressed as $V_{i}\left(\omega_{1}, \omega_{2}, \omega_{3}\right)=c+\zeta_{i}$, where $\zeta_{i}=\frac{2 \Delta}{3} b_{i}(k)$ and

$$
b_{1}(k)=\frac{k^{2} K(k)}{E(k)-K(k)}, \quad b_{2}(k)=\frac{k^{2}\left(1-k^{2}\right) K(k)}{\left(1-k^{2}\right) K(k)-E(k)}, \quad b_{3}(k)=\frac{\left(1-k^{2}\right) K(k)}{E(k)},
$$

with $K(k), E(k)$ as in (4.32), (4.33) denoting elliptic integrals of the first and second kind. In Figure 3 we plot the characteristic velocities in terms of the period $X(k)$ of the underlying $\mathrm{KdV}$ wave train. In particular, we see that for all $k \in(0,1)$ the characteristic velocities are distinct, corresponding to satisfaction of (4.3), i.e. to strict hyperbolicity of the associated Whitham modulation equation. 
Next, we compute the eigenvalues of the relaxed Whitham modulation system (4.22), which is also the limit as $\delta \rightarrow 0$ of the Whitham modulation system associated to (1.1) for fixed $\delta>0$ [NR2]. Recall from Proposition 2.4 that we must restrict ourselves to those cnoidal waves of form (4.30) such that the selection principle $\kappa=\mathcal{G}(k)$ holds. In terms of the $\left(\omega_{1}, k^{2}, \Delta\right)$ parameterization of the KdV Whitham system, this modulation system restricted to the "near-KdV" wave trains discussed in Proposition 2.4 can be expressed as

$$
\partial_{T} \kappa+\kappa \partial_{X} c=0, \quad \partial_{T}\langle u\rangle+\langle u\rangle \partial_{X} c+\partial_{X} a=0,
$$

where

$$
\langle u\rangle=\beta_{1}+\left(k^{2}-1+4 \frac{E(k)}{K(k)}\right) \Delta, \quad a=-\frac{1}{6}\left(3 \beta_{1}^{2}+2\left(k^{2}+1\right) \Delta \beta_{1}-\left(k^{2}-1\right)^{2} \Delta^{2}\right),
$$

and, recalling Proposition 2.4

$$
\kappa=\mathcal{G}(k), \quad \Delta(k)=\frac{3}{2}\left(\frac{K(k) \mathcal{G}(k)}{2 \pi}\right)^{2} .
$$

To compute the eigenvalues of this relaxed modulation system, using the Galilean invariance of (2.1) we require $\langle u\rangle=0$, which is equivalent to requiring $\omega_{1}=$ $-\left(k^{2}-1+4 \frac{E(k)}{K(k)}\right) \Delta(k)$. This reduction thus leaves the elliptic modulus $k$ as the only parameter of the problem. It is then a lengthly but straightforward calculation to show that the eigenvalues $\beta_{i}^{\star}(k), i=1,2$, of (4.34) are given by the roots of the polynomial equation

$$
A(k) \lambda^{2}-B(k) \lambda+C(k)=0,
$$

where the coefficients are given by $A(k)=\mathcal{G}^{\prime}(k)$,

$$
\begin{aligned}
B(k)= & \mathcal{G}^{\prime}(k)\left(k^{2}-1-\frac{k^{2}+1}{3}+\frac{4 E(k)}{K(k)}\right) \Delta(k) \\
& -\mathcal{G}(k)\left[\left(k^{2}-1-\frac{k^{2}+1}{3}+\frac{4 E(k)}{K(k)}\right) \Delta(k)\right]^{\prime}, \\
C(k)= & -\frac{2 \mathcal{G}(k) \Delta(k)\left(2 k^{2}-1\right)}{9}\left(2 k \Delta(k)+\left(k^{2}+1\right) \Delta^{\prime}(k)\right) .
\end{aligned}
$$

Notice that the roots of (4.35) correspond to the eigenvalues $\beta_{j}^{*}$ considered earlier. In Figure 3 we have plotted the characteristic wave speeds $\left\{\alpha_{i}^{*}(X(k))\right\}_{i=1,2,3}$ and $\left\{\beta_{j}^{*}(X(k))\right\}_{j=1,2}$ as functions of the period $X(k)$ of the underlying wave train. From these numerics, it is clear that the subcharacteristic conditions (S1) and (S2) are satisfied for all waves with period $X \geq X_{c}$, where the critical period is $X_{c} \approx 8$. For $X<X_{c}$, condition (S2) is violated, corresponding to a sideband (modulational) instability of the associated wave train. This threshold is consistent with the one found in $[\mathrm{BN}]$. Furthermore, since the low-frequency stability conditions (S1)-(S3) are satisfied for all periods $X \geq X_{c}$, we see also that the upper stability boundary $X \approx 26.17$ cannot be associated with a sideband instability, again consistent with the observations of $[\mathrm{BN}]$.

Finally, we check the subcharacteristic condition (S3) and consider the spatially homogeneous perturbations (independent of the space variable). The Whitham equations read

$$
\partial_{T} \kappa=0, \quad \partial_{T}\langle u\rangle=0, \quad \partial_{T}\left\langle\frac{u^{2}}{2}\right\rangle=\bar{\delta}\left(\left\langle\left(u^{\prime}\right)^{2}\right\rangle-\left\langle\left(u^{\prime \prime}\right)^{2}\right\rangle\right),
$$




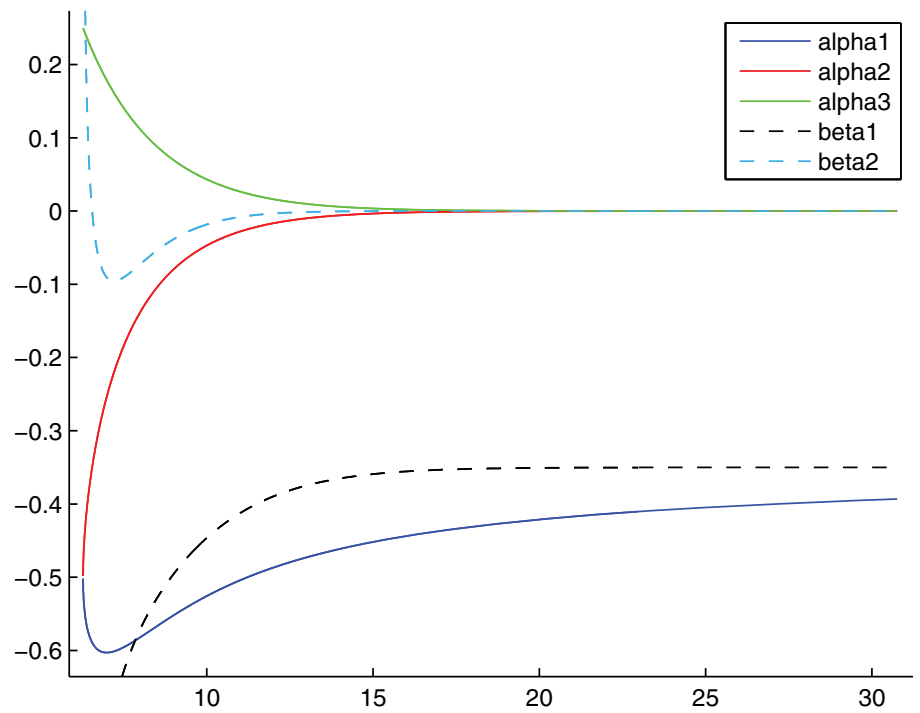

Figure 3. Here, we plot the characteristic velocities $\left\{\alpha_{j}(X(k))\right\}_{j=1}^{3}$ and $\left\{\beta_{j}(X(k))\right\}_{j=1}^{2}$ for the Whitham system for the Korteweg-de Vries equation and the relaxed Whitham's system (4.34), respectively, as functions of the period $X(k)$ of the underlying wave train.

where $u$ is defined by (4.30). In this setting, we use $k, M=\langle u\rangle$ and $\Delta=u_{3}-u_{1}$ as parameters. One thus has

$$
\kappa=\frac{\pi}{K(k)} \sqrt{\frac{\Delta}{3}}, \quad\left\langle\frac{u^{2}}{2}\right\rangle=\frac{M^{2}}{2}-\frac{1}{6} P(k) \Delta^{2},
$$

with $P(k)=1-k^{2}+4\left(k^{2}-2\right) E(k) / K(k)+12(E(k) / K(k))^{2}$. Next, one can show that the source term is written in a simpler form,

$$
R(k, M, \Delta)=\bar{\delta}\left(\left\langle\left(u^{\prime}\right)^{2}\right\rangle-\left\langle\left(u^{\prime \prime}\right)^{2}\right\rangle\right)=r(k, M, \Delta)(\bar{\Delta}(k)-\Delta),
$$

with $r(k, M, \Delta)>0$ and $\bar{\Delta}$ given by

$$
\bar{\Delta}(k)=\frac{21}{20} \frac{2\left(k^{4}-k^{2}+1\right) E(k)-\left(1-k^{2}\right)\left(2-k^{2}\right) K(k)}{\left(-2+3 k^{2}+3 k^{4}-2 k^{6}\right) E(k)+\left(k^{6}+k^{4}-4 k^{2}+2\right) K(k)} .
$$

The steady states of (4.36) correspond to $\Delta=\bar{\Delta}(k)$. By linearizing (4.36) about a steady state $\left(k_{*}, M_{*}, \Delta_{*}=\bar{\Delta}\left(k_{*}\right)\right)$ and searching for solutions that grow in time exponentially, one finds the dispersion relation

$$
\Lambda^{2}\left(\Lambda-\Lambda_{*}\right)=0
$$

with $\Lambda_{*}$ satisfying

$$
\left(\left(\frac{P\left(k_{*}\right) K^{\prime}\left(k_{*}\right)}{3 K\left(k_{*}\right)}+\frac{P^{\prime}\left(k_{*}\right)}{12}\right) \Delta_{*}^{2}\right) \Lambda_{*}=r\left(k_{*}, M_{*}, \Delta_{*}\right)\left(\frac{\Delta_{*} K^{\prime}\left(k_{*}\right)}{K\left(k_{*}\right)}-\frac{\bar{\Delta}^{\prime}\left(k_{*}\right)}{2}\right) .
$$




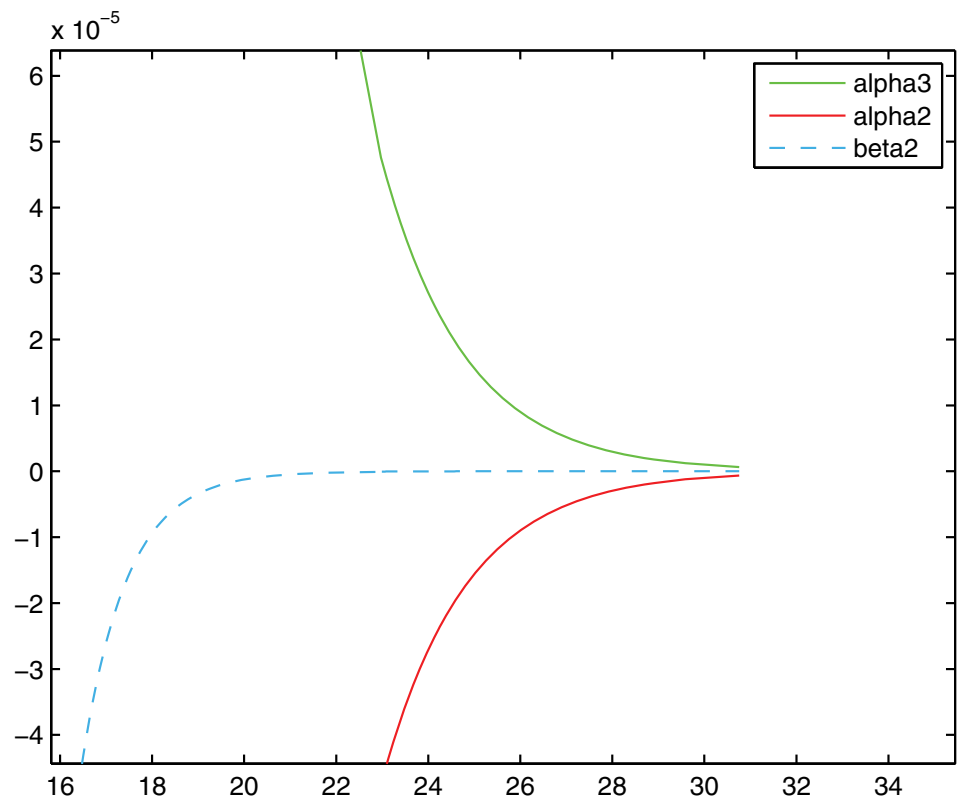

Figure 4. Here, we zoom in on the largest eigenvalues $\alpha_{2,3}(X(k))$ and $\beta_{2}(X(k))$ in Figure 3, noting in particular that $\alpha_{2}(X(k))<$ $\beta_{2}(X(k))<\alpha_{3}(X(k))$ for all periods $X(k) \geq X_{c}$ of the underling wave train.

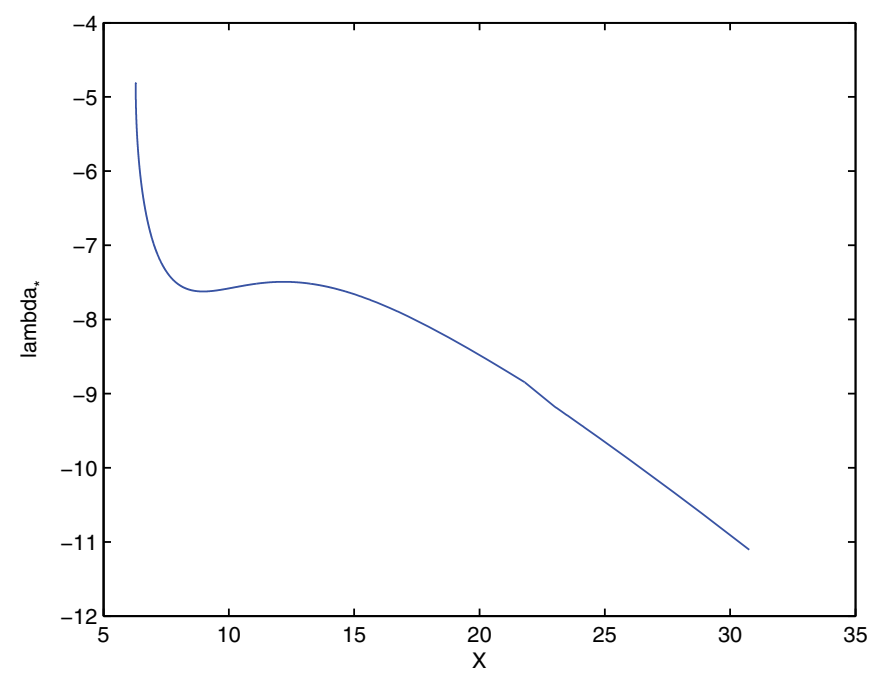

Figure 5. Here, we plot $\lambda_{*}(X(k))$ as a function of the period $X(k)$ of the underling wave train.

The subcharacteristic condition (S3) is satisfied if and only if $\Lambda_{*}<0$. In Figure [5] we have represented $\lambda_{*}=\Lambda_{*} / r\left(k_{*}, M_{*}, \bar{\Delta}_{*}\right)$ as a function of the period $X$. We 
clearly see that the subcharacteristic condition (S3) is always satisfied on the range of period $\left[2 \pi, X_{m}\right]$ with $X_{m} \geq 30$. In particular, (S3) holds for all near-KdV wave trains with period $X \geq X_{c}$, corresponding to the low-frequency stability boundary, and $X \leq 26.17$, corresponding to the high-frequency stability boundary computed in $\mathrm{BN}$.

\section{Appendix A. High-Frequency spectra: The computations of BN}

In this appendix, we review the formal expansions of [BN], upon which condition (A3) is based. In particular, we rigorously justify the formal calculations contained therein by using Corollary 3.8 to expand all the high-frequency eigenvalues of the $\mathrm{KdV}-\mathrm{KS}$ equation for $0<\delta \ll 1$, showing that, in our rigorous framework, we recover exactly the formulas in $[\mathrm{BN}]$ for $\Re\left(\lambda_{1}\left(\lambda_{0}, \xi\right)\right)$ when $\lambda_{0} \neq 0$ is an eigenvalue of some (Bloch) linearized $\mathrm{KdV}$ operator $L_{\mathrm{KdV}, \xi}$. In this way, we provide justification that condition (A3) is meaningful and has indeed been numerically investigated in BN].

A.1. Formal asymptotics as $\delta \rightarrow 0$. To begin, we fix $X \in \mathcal{W}$ and let $u_{\delta}$ be an $X$-periodic traveling wave solution of (1.1), defined for all $\delta \in\left(0, \delta_{0}\right)$ for some $\delta_{0}>0$ sufficiently small. Recall that in the proof of Proposition 3.7 we obtained an asymptotic expansion for $0<\delta \ll 1$ of the periodic Evans function associated with the linearization of (1.1) about $u_{\delta}$ up to $\mathcal{O}\left(\delta^{3}\right)$. However, an explicit expansion of the eigenvalues of such a spectral problem is often complicated to obtain by using analytic Evans function techniques. As an alternative, recalling the notation in (1.4), here we fix a Bloch wave number $\xi \in[-\pi / X, \pi / X)$ and a non-zero eigenvalue $\lambda_{0}$ of $L_{\mathrm{KdV}, \xi}\left[u_{0}\right] k$ and search, for $0<\delta \ll 1$, directly for an expansion of the $L^{\infty}(\mathbb{R})$ eigenvalues $\lambda\left(\xi, \lambda_{0}, \delta\right)$ and eigenfunctions $v\left(\cdot ; \xi, \lambda_{0}, \delta\right)$ of $L_{\xi}\left[u_{\delta}\right]$ in the form

$$
\left\{\begin{array}{l}
\lambda\left(\xi, \lambda_{0}, \delta\right)=\lambda_{0}+\delta \lambda_{1}\left(\xi, \lambda_{0}\right)+\delta^{2} \lambda_{2}\left(\xi, \lambda_{0}\right)+\mathcal{O}\left(\delta^{3}\right), \\
v\left(\cdot ; \xi, \lambda_{0}, \delta\right)=v_{0}\left(\cdot ; \xi, \lambda_{0}\right)+\delta v_{1}\left(\cdot ; \xi, \lambda_{0}\right)+\delta^{2} v_{2}\left(\cdot ; \xi, \lambda_{0}\right)+\mathcal{O}\left(\delta^{3}\right)
\end{array}\right.
$$

Note that such expansions are guaranteed to exist by Corollary 3.8 and the Dunford Calculus.

Now, recall that the spectral problem (3.1) for the operator $L\left[u_{\delta}\right]$ can be written as

$$
\left\{\begin{array}{l}
v^{\prime \prime \prime}+\left(\left(u_{\delta}-c\right) v\right)^{\prime}+\delta\left(v^{\prime \prime}+v^{\prime \prime \prime \prime}\right)+\lambda v=0, \\
v(x+X)=e^{i \xi X} v(x)
\end{array}\right.
$$

with $v \in L_{\text {per }}^{2}([0, X])$. For $\delta=0$, it is known by the results of $[\mathrm{BD}$ that the spectrum lies on the imaginary axis and is parameterized by

$$
\Im \lambda= \pm 8 \sqrt{\left|\eta-\eta_{1}\right|\left|\eta-\eta_{2}\right|\left|\eta-\eta_{3}\right|}, \quad \eta \in\left(-\infty, \eta_{1}\right] \cup\left[\eta_{2}, \eta_{3}\right],
$$

where $\eta_{1}=k^{2}-1, \eta_{2}=2 k^{2}-1$ and $\eta_{3}=k^{2}$ and $k$ is the elliptic modulus associated with the underlying elliptic function solution $u_{0}$ of the KdV equation for this particular period $X$. Moreover, the Bloch wave number $\xi$ can be written as

$$
\xi=\frac{N \pi}{2 K(k)} \pm \frac{\sqrt{\left|\eta-\eta_{1}\right|\left|\eta-\eta_{2}\right|\left|\eta-\eta_{3}\right|}}{K(k)} \int_{0}^{K(k)} \frac{d y}{\eta-k^{2}+\operatorname{dn}(y, k)}
$$

for some $N \in \mathbb{N}$. 
Before beginning our analysis of the perturbation expansion (A.1), we make some preliminary remarks concerning the spectrum of the linearized KdV operator. Let

$$
L_{\mathrm{KdV}}=-\partial_{x}\left(u_{0}-c_{0}\right)-\partial_{x}^{3}
$$

denote the linearized $\mathrm{KdV}$ operator, considered as a closed densely defined operator on $L^{2}(\mathbb{R})$, and let $\left(L_{\mathrm{KdV}, \xi}\right)_{\xi \in[-\pi / X, \pi / X)}$ denote the associated family of Bloch operators defined on $L_{\text {per }}^{2}([0, X])$. By the results of $[\mathrm{BD}], \sigma\left(L_{\mathrm{KdV}}\right)=i \mathbb{R}$, corresponding to spectral stability of the underlying cnoidal wave solution $u_{0}$. Furthermore, when condition (A1) holds 17 given any $\xi \in[-\pi / X, \pi / X)$ the non-zero eigenvalues of the $\mathrm{KdV}$ Bloch operators $L_{\mathrm{KdV}, \xi}$ are simple. Notice one may be easily misled by the fact that, in some sense, each $\lambda \in i \mathbb{R} \backslash\{0\}$ lies in the spectrum of $L_{\mathrm{KdV}}$ with multiplicity either 1,2 , or 3 , since there exists either a unique $\xi \in[-\pi / X, \pi / X)$ such that $\lambda \in \sigma\left(L_{\mathrm{KdV}, \xi}\right)$ (corresponding to multiplicity 1 ) or else there exist either twd $\sqrt{18}$ or three distinct such $\xi$ (corresponding to multiplicity 2 and 3, respectively); see $[\mathrm{BD}]$ for details. However, assuming the period of the underlying wave satisfies condition (A1), when expanding such eigenvalues for a fixed $\xi$ one is doing simple perturbation theory. On the other hand, $\lambda=0$ is known to be an eigenvalue of the $\mathrm{KdV}$ Bloch operator $L_{\mathrm{KdV}, 0}$, corresponding to $\xi=0$, with algebraic multiplicity three and geometric multiplicity two. Indeed, one can easily verify that $\operatorname{Ker}\left(L_{\mathrm{KdV}, 0}\right)$ is two dimensional and $1 \in \operatorname{Ker}\left(L_{\mathrm{KdV}, 0}^{2}\right)$; see $\mathrm{BrJ}, \mathrm{BrJK}$ for details. Thus, a separate analysis is indeed necessary when considering the bifurcation of the neutral modes of $L_{\mathrm{KdV}, 0}$ for $0<\delta \ll 1$.

We now begin our perturbation analysis by fixing $\xi \in[-\pi / X, \pi / X)$ and considering the continuation of a fixed non-zero eigenvalue $\lambda_{0}$ of $L_{\mathrm{KdV}, \xi}$. To this end, let $v_{0} \in L_{\text {per }}^{2}([0, X])$ be a non-trivial function in the null-space of the operator $L_{\mathrm{KdV}, \xi}-\lambda_{0} I$ and insert the expansions (2.12) and (A.1) into (A.2). Collecting the $\mathcal{O}\left(\delta^{0}\right)$ terms we find that $v_{0}$ must satisfy

$$
\left\{\begin{array}{l}
v_{0}^{\prime \prime \prime}+\left(\left(u_{0}-c_{0}\right) v_{0}\right)^{\prime}+\lambda_{0} v_{0}=0 \\
v_{0}(x+X)=e^{i \xi X} v_{0}(x)
\end{array}\right.
$$

which clearly holds by our choice of $\left(\lambda_{0}, v_{0}\right)$. Continuing the expansion, identifying the $\mathcal{O}\left(\delta^{1}\right)$ terms implies that $v_{1}\left(\cdot ; \xi, \lambda_{0}\right)$ and $\lambda_{1}\left(\xi, \lambda_{0}\right)$ must satisfy

$$
\left\{\begin{array}{l}
v_{1}^{\prime \prime \prime}+\left(\left(u_{0}-c_{0}\right) v_{1}\right)^{\prime}+\lambda_{0} v_{1}+\lambda_{1} v_{0}+\left(U_{1} v_{0}\right)^{\prime}+v_{0}^{\prime \prime}+v_{0}^{\prime \prime \prime \prime}=0, \\
v_{1}(x+X)=e^{i \xi X} v_{1}(x),
\end{array}\right.
$$

where here the function $U_{1}$ is defined as in Proposition 2.4. To analyze the solvability of (A.3) we consider the operator $\widehat{L_{\mathrm{KdV}, \xi}}=\partial_{x}^{3}+\partial_{x}\left(u_{0}-c_{0}\right)$ defined on all functions $v \in H^{3}(0, X)$ such that $v(x+X)=e^{i \xi_{j} X} v(x)$, and note then that the operator $\widetilde{L_{\mathrm{KdV}, \xi}}+\lambda_{0} I$ is Fredholm of index 0 on $H^{3}(0, X)$. In particular, we have Range $\left.\left(\widetilde{L_{\mathrm{KdV}, \xi}}+\lambda_{0} I\right)=\operatorname{Ker}\left(\widehat{\left(\widetilde{L_{\mathrm{KdV}, \xi}}\right.}+\lambda_{0} I\right)^{*}\right)^{\perp}$, where the adjoint operator of $\widetilde{L_{\mathrm{KdV}, \xi}}+\lambda_{0} I$ is given by

$$
\left(\widetilde{L_{\mathrm{KdV}, \xi}}+\lambda_{0} I\right)^{*}=-\partial_{x}^{3}-\left(u_{0}-c_{0}\right) \partial_{x}-\lambda_{0} I,
$$

\footnotetext{
${ }^{17}$ We recall that it is justified numerically for some given waves in both $\mathrm{BD}$ and Figure 1

${ }^{18}$ The points of multiplicity two correspond to the "turning points" of the triply covered region of the KdV spectrum surrounding the origin. That is, they are found at the endpoints of the triply covered region. See Figure 1
} 
defined here for all $v \in H^{3}(0, X)$ such that $v(x+X)=e^{i \xi_{j} X} v(x)$. Notice that since $\lambda_{0} \neq 0$ we easily identify $\operatorname{Ker}\left(\widetilde{L_{\mathrm{KdV}, \xi}}+\lambda_{0} I\right)^{*}$. Indeed, as is easily verified, the function

$$
w_{0}(x)=\int_{x}^{x+X} v_{0}(s) d s
$$

is non-trivial, satisfies the boundary condition $w_{0}(x+X)=e^{i \xi X} w_{0}(x)$, and lies in

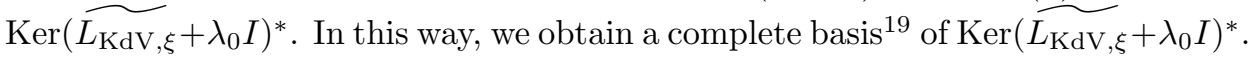

Now, with the above notation it follows by the Fredholm alternative that equation (A.3) has a solution provided the compatibility condition

$$
\left\langle\lambda_{1} v_{0}+\left(U_{1} v_{0}\right)^{\prime}+v_{0}^{\prime \prime}+v_{0}^{\prime \prime \prime \prime} ; w_{0}\right\rangle=0
$$

is satisfied, where here $\langle\cdot, \cdot\rangle$ denotes the standard (sesquilinear) inner product on $L_{\text {per }}^{2}([0, X])$. We now give an expression for $\lambda_{1}$ with respect to functions $w_{0}$. To this end, note that by definition we have the identity

$$
w_{0}^{\prime}(x)=v_{0}(x+X)-v_{0}(x)=\left(e^{i \xi X}-1\right) v_{0}(x),
$$

from which it follows that

$$
\left\langle v_{0}, w_{0}\right\rangle=\frac{1}{e^{i \xi X}-1} \int_{0}^{X} w_{0}^{\prime} \bar{w}_{0} d x=\frac{e^{-i \xi X / 2}}{2 \sin (\xi X / 2)} \Im\left(\int_{0}^{X} w_{0}^{\prime} \bar{w}_{0} d x\right) .
$$

Similar computations yield the following identities:

$$
\begin{aligned}
& \left\langle\left(U_{1} v_{0}\right)^{\prime} ; w_{0}\right\rangle=2 i e^{-i \xi X / 2} \sin (\xi X / 2) \int_{0}^{X} U_{1}\left|v_{0}\right|^{2} d x, \\
& \left\langle v_{0}^{\prime \prime} ; w_{0}\right\rangle=-2 e^{-i \xi X / 2} \sin (\xi X / 2) \Im\left(\int_{0}^{X} v_{0}^{\prime} \bar{v}_{0} d x\right), \\
& \left\langle v_{0}^{\prime \prime \prime \prime} ; w_{0}\right\rangle=2 e^{-i \xi X / 2} \sin (\xi X / 2) \Im\left(\int_{0}^{X} v_{0}^{\prime \prime} \bar{v}_{0}^{\prime} d x\right) .
\end{aligned}
$$

Taking real and imaginary parts of (A.5), we find

$$
\begin{aligned}
& \Im\left(\int_{0}^{X} w_{0}^{\prime} \bar{w}_{0} d x\right) \Re\left(\lambda_{1}\right)=\Im\left(\int_{0}^{X} w_{0}^{\prime \prime} \bar{w}_{0}^{\prime}-w_{0}^{\prime \prime \prime} \bar{w}_{0}^{\prime \prime} d x\right), \\
& \Im\left(\int_{0}^{X} w_{0}^{\prime} \bar{w}_{0} d x\right) \Im\left(\lambda_{1}\right)=-\int_{0}^{X} U_{1}\left|w_{0}^{\prime}\right|^{2} d x .
\end{aligned}
$$

Note that, up to $\mathcal{O}(\delta)$, the $\mathcal{O}(\delta)$ correction $U_{1}$ of the underlying periodic profile $U$ can only contribute to the imaginary part of $\lambda$. Furthermore, this contribution actually vanishes, as is seen from parity: indeed, note that

$$
\left|w_{0}^{\prime}\right|^{2}=\sin ^{2}(\xi X / 2)\left(1+\left|\lambda_{0}^{-1} u_{0}^{\prime}\right|^{2}\right)
$$

is an even function whereas, by Proposition 2.4, $U_{1}$ is an odd function. As these functions are both $2 X$-periodic, assuming that $20 \int_{0}^{X} w_{0}^{\prime} \bar{w}_{0} d x \neq 0$, the integral which defines $\Im\left(\lambda_{1}\right)$ then vanishes, implying that $\Im\left(\lambda_{1}\right)=0$. As a result, we have

\footnotetext{
${ }^{19}$ Notice that when $\lambda_{0}=0$, corresponding to $\xi=0$ by (A1), the above construction yields only constant functions; indeed, one readily finds as a consequence of the conservative structure of the $\mathrm{KdV}$ equation (2.1) that $1 \in \mathrm{Ker} \widehat{\mathrm{LdV}_{\mathrm{Kd}}} *$.

${ }^{20}$ The non-vanishing of this integral is part of the numerical investigation of condition (A3), as carried out in $[\mathrm{BN}]$, but was seen there to not be an issue.
} 
obtained an expansion valid up to order $\mathcal{O}\left(\delta^{2}\right)$ for any eigenvalue $\lambda\left(\xi, \lambda_{0}, \delta\right)$ such that $\lambda_{0} \neq 0$ and $\int_{0}^{X} w_{0}^{\prime} \bar{w}_{0} d x \neq 0$. Furthermore, we find that

$$
\Re\left(\lambda_{1}\left(\xi, \lambda_{0}\right)\right)=\frac{\Im\left(\int_{0}^{X} w_{0}^{\prime \prime} \bar{v}_{0}^{\prime}-w_{0}^{\prime \prime \prime} \bar{w}_{0} d x\right)}{\Im\left(\int_{0}^{X} w_{0}^{\prime} \bar{w}_{0} d x\right)},
$$

which is precisely the formula determined in the formal analysis of $[\mathrm{BN}]$. In particular, in $\mathrm{BN}$ the above quantity was computed numerically and was found to be strictly negative for all $\xi \in[-\pi / X, \pi / X)$ and $\lambda \in \mathbb{R} i \backslash\{0\}$ provided the period $X$ of the underlying wave $u_{\delta}$ satisfies $X \in\left(X_{1}, X_{2}\right)$ where $X_{1} \approx 8.49$ and $X_{2} \approx 26.17$; see Section A.2 below for a summary of the numerical scheme used in [BN].

Remark A.1. Using similar methods to those discussed above, one can also derive an expansion for the triple eigenvalue $\lambda_{0}=0$ for the linearized $\mathrm{KdV}$ operator $L_{\mathrm{KdV}, 0}$ with $\xi=0$ held fixed and $0<\delta \ll 1$. Indeed, one can show that near the origin the operator $L_{0}\left[u_{\delta}\right]$ has $\lambda=0$ as an eigenvalue with algebraic multiplicity two, and a third eigenvalue $\lambda_{\text {crit }}(\delta)$ that can be expanded analytically with respect to $\delta$ for $\delta \ll 1$ with

$$
\Re\left(\lambda_{\text {crit }}^{\prime}(0)\right)=-\frac{\left\langle\left(U_{1} \psi\right)^{\prime}+\psi_{2}^{\prime \prime}+\psi_{2}^{\prime \prime \prime \prime}, u_{0}\right\rangle}{\left\langle\psi_{2}, u_{0}\right\rangle} \in \mathbb{R},
$$

where $u_{0}$ and $U_{1}$ are as in Proposition 2.4 and

$$
\psi=1-\left(\partial_{k} c_{0}\right)^{-1} \partial_{k} u_{0} .
$$

See the appendix of $[\mathrm{BN}]$ for details. Notice that, by Lemma 4.7, this gives an explicit definition of the constant $\gamma$ introduced in Proposition 4.1 .

A.2. Numerical computations. In this appendix, we describe in our own notation the numerical computations carried out in $[\mathrm{BN}]$ determining the sign of the real part of the $\mathcal{O}(\delta)$ corrector $\lambda_{1}\left(\xi, \lambda_{0}\right)$ for a fixed $\xi \in[-\pi / X, \pi / X)$ and a non-zero eigenvalue $\lambda_{0} \in \sigma\left(L_{\mathrm{KdV}, \xi}\left[u_{0}\right]\right)$. To this end, recall that for a fixed $\xi \in[-\pi / X, \pi / X)$ the $L^{\infty}(\mathbb{R})$ eigenvalues $\lambda\left(\xi, \lambda_{0}, \delta\right)$ can be expanded for $0<\delta \ll 1$ as in (A.1), where we recall that for $\lambda_{0} \neq 0$ the real part of the $\mathcal{O}(\delta)$ corrector can be found from (A.6), assuming $\int_{0}^{X} w_{0}^{\prime} \bar{w}_{0} d x \neq 0$, as

$$
\Re\left(\lambda_{1}\right)=\frac{\Im\left(\int_{0}^{X} w_{0}^{\prime \prime} \bar{w}_{0}^{\prime}-w_{0}^{\prime \prime \prime} \bar{w}_{0}^{\prime \prime}\right)}{\Im\left(\int_{0}^{X} w_{0}^{\prime} \bar{w}_{0}\right)}=\frac{\left\langle w_{0}^{\prime}, w_{0}^{\prime \prime}+w_{0}^{\prime \prime \prime \prime}\right\rangle}{\left\langle w_{0}^{\prime}, w_{0}\right\rangle} ;
$$

notice that this is precisely formula (54) on page 593, with $\Phi_{0}=w_{0}$, for the $\mathcal{O}(\delta)$ correction of non-zero KdV eigenvalues $\lambda_{0}$ found in [BN]. Using Mathematica, the authors of $[\mathrm{BN}]$ then numerically evaluate the quantity

$$
\sup _{\substack{\xi \in[-\pi / X, \pi / X) \\ \lambda_{0} \in \sigma\left(L_{\mathrm{KdV}, \xi}\left[u_{0}\right]\right) \backslash\{0\}}} \Re\left(\lambda_{1}\left(\xi, \lambda_{0}\right)\right),
$$

which clearly must be non-positive to prevent instability. The details of these computations are as follows.

First, denote

$$
\omega=\frac{\pi}{\kappa}, \quad \omega^{\prime}=\frac{K\left(\sqrt{1-k^{2}}\right) \pi}{K(k) \kappa} .
$$


Following the stability analysis for the $\mathrm{KdV}$ equation (2.1) presented in $[\mathrm{Sp}$, the authors of $[\mathrm{BN}]$ parameterize the eigenvalues and eigenfunctions $\lambda_{0}$ and $\hat{v}_{0}$ and the Bloch wave number $\xi$ as

$$
\hat{v}_{0}(x)=\frac{\sigma^{2}\left(x+i \omega^{\prime}+\alpha\right)}{\sigma^{2}\left(x+i \omega^{\prime}\right) \sigma^{2}(\alpha)} e^{-2\left(x+i \omega^{\prime}\right) \zeta(\alpha)}, \quad \lambda_{0}=-4 \nu^{\prime}(\alpha), \quad \xi=2 i\left(\zeta(\alpha)-\frac{\alpha}{\omega} \zeta(\omega)\right),
$$

where here $\sigma$ and $\zeta$ denote Weierstrass's sigma- and zeta-functions, respectively, $\nu(z)$ denotes the Weierstrass elliptic function with periods $\omega=\frac{\pi}{\kappa}$ and $i \omega^{\prime}$ where $\omega^{\prime}=\frac{K\left(\sqrt{1-k^{2}}\right) \pi}{\kappa K(k)}$. Notice that $\xi \in \mathbb{R}$ only if $\Re(\alpha)=n \omega, n \in \mathbb{N}$. In this case, the problem is parameterized by $\alpha$ and $k$, since $\kappa$ is determined by the selection criterion $\kappa=\widetilde{\mathcal{G}}(k)$ given by formula $(34)$ on page 590 in $[\mathrm{BN}$. In $[\mathrm{BN}$, the authors described the computations for $\alpha=n \omega+i \beta$ for $n=0,1$ and $\beta \in[0,2 \omega]$, claiming that the other cases $n \geq 2$ do not provide any new results. There, the parameter $k$ was restricted to the interval $\left[0,1-10^{-7}\right]$, which corresponds to periods $X=\frac{2 \pi}{\kappa}$ lying approximately in the interval $[2 \pi, 10 \pi]$. In order to evaluate the Weierstrass elliptic functions, the usual theta functions are used:

$$
\begin{aligned}
& \Theta(z)=2 \sum_{n=1}^{\infty}(-1)^{n+1} q_{0}^{(2 n+1)^{4} / 4} \sin ((2 n-1) \pi z / 2 K(k)), \\
& \Theta_{1}(z)=2 \sum_{n=1}^{\infty}(-1)^{n+1} q_{0}^{(2 n+1)^{4} / 4} \cos ((2 n-1) \pi z / 2 K(k)),
\end{aligned}
$$

with $q_{0}=\exp \left(-\pi K\left(\sqrt{1-k^{2}}\right) / K(k)\right)$. Then the various Weierstrass functions are represented as

$$
\begin{aligned}
& \nu(z)=e_{1}+\lambda\left(\frac{\Theta_{1}(z \sqrt{\lambda}) \Theta^{\prime}(0)}{\Theta_{1}(0) \Theta(z \sqrt{\lambda})}\right)^{2}, \\
& \zeta(z)=\zeta(\omega) \frac{z}{\omega}+\sqrt{\lambda} \frac{\Theta^{\prime}(z \sqrt{\lambda})}{\Theta(z \sqrt{\lambda})}, \\
& \sigma(z)=\frac{1}{\sqrt{\lambda}} \exp \left(\frac{\zeta(\omega) z^{2}}{2 \omega}\right) \frac{\Theta(z \sqrt{\lambda})}{\Theta^{\prime}(0)},
\end{aligned}
$$

where $e_{1}=\nu(\omega), \lambda=\nu(\omega)-\nu\left(\omega+i \omega^{\prime}\right)$.

Using the above approach, it is numerically demonstrated in $\mathrm{BN}$ that the quantity

$$
\sup _{\substack{\xi \in[-\pi / X, \pi / X) \\ \lambda_{0} \in \sigma\left(L_{\mathrm{KdV}, \xi}\left[u_{0}\right]\right) \backslash\{0\}}} \Re\left(\lambda_{1}\left(\xi, \lambda_{0}\right)\right)
$$

is strictly negative for all periods in the interval $[8.49,26.17]$. In particular, notice that from Figure 3 the subcharacteristic conditions (S1)-(S3) hold in this interval, as indicated in Section 4.1. Furthermore, the left stability boundary corresponds to $\xi \approx 0$, hence to a sideband-type instability; as noted in the previous section, the right stability boundary does not. For each $k$, and thus each period, the authors determine approximately the value $\xi_{m}$ where the functions $\xi \mapsto \sup _{\lambda_{0} \in \sigma\left(L_{\mathrm{KdV}, \xi}\left[u_{0}\right]\right) \backslash\{0\}} \Re \lambda_{1}\left(\xi, \lambda_{0}\right)$ take their maximal values, which provides the boundaries of the stability region (in the period). 
As mentioned throughout our analysis, it is important to note that the analysis of $[\mathrm{BN}]$ a priori explores regions where the eigenvalues expand as

$$
\lambda\left(\xi, \lambda_{0}, \delta\right)=\lambda_{0}+\delta \lambda_{1}\left(\xi, \lambda_{0}\right)+\mathcal{O}\left(\delta^{2}\right),
$$

and is thus limited only to some particular regions of the $(|\xi|, \delta)$-plane. In particular, we stress that only the unveiling of the role of subcharacteristic conditions enables us to prove that, though from the analysis of $[\mathrm{BN}$ it is not possible to conclude spectral stability, their numerical investigation is still sufficient to complete our analysis.

Finally, we note that another way of carrying out these computations would be to instead use the parameterization of eigenvalues and eigenvectors presented in BD. In this case, one has

$$
\begin{gathered}
\left.\left.\lambda_{0}(\eta)= \pm 8 i \sqrt{\left|\eta-\eta_{1}\right|\left|\eta-\eta_{2}\right|\left|\eta-\eta_{3}\right|}, \quad \eta \in\right]-\infty, \eta_{1}\right] \cup\left[\eta_{2}, \eta_{3}\right], \\
\xi=\frac{N \pi}{2 K(k)} \pm \frac{8 \sqrt{\left|\eta-\eta_{1}\right|\left|\eta-\eta_{2}\right|\left|\eta-\eta_{3}\right|}}{K(k)} \int_{0}^{K(k)} \frac{d y}{\eta-k^{2}+\operatorname{dn}(y, k)} \\
w_{0}(x)=\int_{x}^{x+X(k)}\left(\lambda_{0}(\eta)-\frac{u_{0}^{\prime}(y)}{3}\right) \exp \left(-\int_{0}^{y} \frac{\lambda_{0}(\eta) d z}{u_{0}(z) / 3-c_{0}+\eta}\right) d y
\end{gathered}
$$

with $\eta_{1}=k^{2}-1, \eta_{2}=2 k^{2}-1, \eta_{3}=k^{2}$ and $u_{0}$ the cnoidal wave given by setting $\kappa=\mathcal{G}(k)$ as defined in Proposition 2.4

\section{ACKNOWLEDGEMENTS}

Thanks to Blake Barker for the aid of his numerical Evans function computations carried out for $\delta \ll 1$, some but not all of which appear in BJNRZ1. Also, we thank the anonymous referee for a careful reading of the paper and for helpful comments and suggestions that greatly improved the exposition.

\section{REFERENCES}

[BN] Doron E. Bar and Alexander A. Nepomnyashchy, Stability of periodic waves governed by the modified Kawahara equation, Phys. D 86 (1995), no. 4, 586-602, DOI 10.1016/0167-2789(95)00174-3. MR1353179 (96m:35018)

[BHZ] Blake Barker, Jeffrey Humpherys, and Kevin Zumbrun, One-dimensional stability of parallel shock layers in isentropic magnetohydrodynamics, J. Differential Equations 249 (2010), no. 9, 2175-2213, DOI 10.1016/j.jde.2010.07.019. MR.2718655 (2011j:76088)

[BD] Nate Bottman and Bernard Deconinck, KdV cnoidal waves are spectrally stable, Discrete Contin. Dyn. Syst. 25 (2009), no. 4, 1163-1180, DOI 10.3934/dcds.2009.25.1163. MR:2552133 (2011h:35239)

[B] B. Barker, Numerical proof of stability of roll waves in the small-amplitude limit for inclined thin film flow, to appear, J. Diff. Eq.

[BJNRZ1] Blake Barker, Mathew A. Johnson, Pascal Noble, L. Miguel Rodrigues, and Kevin Zumbrun, Nonlinear modulational stability of periodic traveling-wave solutions of the generalized Kuramoto-Sivashinsky equation, Phys. D 258 (2013), 11-46, DOI 10.1016/j.physd.2013.04.011. MR.3079606

[BJNRZ2] Blake Barker, Mathew A. Johnson, Pascal Noble, L. Miguel Rodrigues, and Kevin Zumbrun, Stability of periodic Kuramoto-Sivashinsky waves, Appl. Math. Lett. 25 (2012), no. 5, 824-829, DOI 10.1016/j.aml.2011.10.026. MR2888080 
[BJNRZ3] B. Barker, M. A. Johnson, P. Noble, L. M. Rodrigues, and K. Zumbrun, Whitham averaged equations and modulational stability of periodic solutions of hyperbolicparabolic balance laws. Proceedings and seminars, Centre de Mathématiques de l'École Polytechnique; Conference proceedings, "Journées équations aux dérivées partielles", 2010, Port d'Albret, France. Available online at: http://jedp.cedram.org/jedpbin/fitem?id=JEDP_2010

[BJRZ] Blake Barker, Mathew A. Johnson, L. Miguel Rodrigues, and Kevin Zumbrun, Metastability of solitary roll wave solutions of the St. Venant equations with viscosity, Phys. D 240 (2011), no. 16, 1289-1310, DOI 10.1016/j.physd.2011.04.022. MR.2813829 (2012h:76039)

[BrJ] Jared C. Bronski and Mathew A. Johnson, The modulational instability for a generalized Korteweg-de Vries equation, Arch. Ration. Mech. Anal. 197 (2010), no. 2, 357-400, DOI 10.1007/s00205-009-0270-5. MR2660515 (2012f:35456)

[BrJK] Jared C. Bronski, Mathew A. Johnson, and Todd Kapitula, An index theorem for the stability of periodic travelling waves of Korteweg-de Vries type, Proc. Roy. Soc. Edinburgh Sect. A 141 (2011), no. 6, 1141-1173, DOI 10.1017/S0308210510001216. MR 2855892

[CD] Hsueh-Chia Chang and Evgeny A. Demekhin, Complex wave dynamics on thin films, Studies in Interface Science, vol. 14, Elsevier Science B.V., Amsterdam, 2002. MR2362445 (2008i:76084)

[CDK] H. C. Chang, E. A. Demekhin, and D. I. Kopelevich, Laminarizing effects of dispersion in an active-dissipative nonlinear medium, Phys. D 63 (1993), 299-320.

[DK] Bernard Deconinck and Todd Kapitula, The orbital stability of the cnoidal waves of the Korteweg-de Vries equation, Phys. Lett. A 374 (2010), no. 39, 4018-4022, DOI 10.1016/j.physleta.2010.08.007. MR.2683991 (2011f:35297)

[DSSS] Arjen Doelman, Björn Sandstede, Arnd Scheel, and Guido Schneider, The dynamics of modulated wave trains, Mem. Amer. Math. Soc. 199 (2009), no. 934, viii+105, DOI 10.1090/memo/0934. MR 2507940 (2011d:35238)

[EMR] N. M. Ercolani, D. W. McLaughlin, and H. Roitner, Attractors and transients for a perturbed periodic KdV equation: a nonlinear spectral analysis, J. Nonlinear Sci. 3 (1993), no. 4, 477-539, DOI 10.1007/BF02429875. MR1246704 (94j:35155)

[FreS] H. Freistühler and P. Szmolyan, Spectral stability of small shock waves, Arch. Ration. Mech. Anal. 164 (2002), no. 4, 287-309, DOI 10.1007/s00205-002-0215-8. MR.1933630 (2003j:35273)

[FST] Uriel Frisch, Zhen-Su She, and Olivier Thual, Viscoelastic behaviour of cellular solutions to the Kuramoto-Sivashinsky model, J. Fluid Mech. 168 (1986), 221-240, DOI 10.1017/S0022112086000356. MR861201 (87j:80015)

[G] R. A. Gardner, On the structure of the spectra of periodic travelling waves, J. Math. Pures Appl. (9) 72 (1993), no. 5, 415-439. MR.1239098 (94j:35072)

[Go1] Jonathan Goodman, Nonlinear asymptotic stability of viscous shock profiles for conservation laws, Arch. Rational Mech. Anal. 95 (1986), no. 4, 325-344, DOI 10.1007/BF00276840. MR853782 (88b:35127)

[Go2] Jonathan Goodman, Remarks on the stability of viscous shock waves, Viscous profiles and numerical methods for shock waves (Raleigh, NC, 1990), SIAM, Philadelphia, PA, 1991, pp. 66-72. MR1142641

[HLZ] Jeffrey Humpherys, Olivier Lafitte, and Kevin Zumbrun, Stability of isentropic NavierStokes shocks in the high-Mach number limit, Comm. Math. Phys. 293 (2010), no. 1, 1-36, DOI 10.1007/s00220-009-0885-2. MR2563797(2010i:76085)

[JZB] Mathew A. Johnson, Kevin Zumbrun, and Jared C. Bronski, On the modulation equations and stability of periodic generalized Korteweg-de Vries waves via Bloch decompositions, Phys. D 239 (2010), no. 23-24, 2057-2065, DOI 10.1016/j.physd.2010.07.012. MR2733113 (2011g:35351)

[JNRZ1] M. A. Johnson, P. Noble, L. M. Rodrigues, and K. Zumbrun, Behaviour of periodic solutions of viscous conservation laws under localized and nonlocalized perturbations, Invent. Math. 197 (2014), no. 1, 115-213.

[JZ1] M. A. Johnson and K. Zumbrun, Rigorous justification of the Whitham modulation equations for the generalized Korteweg-de Vries equation, Stud. Appl. Math. 
125 (2010), no. 1, 69-89, DOI 10.1111/j.1467-9590.2010.00482.x. MR2676781 (2011h:35249)

[JZ2] Mathew A. Johnson and Kevin Zumbrun, Transverse instability of periodic traveling waves in the generalized Kadomtsev-Petviashvili equation, SIAM J. Math. Anal. 42 (2010), no. 6, 2681-2702, DOI 10.1137/090770758. MR2733265 (2011k:35199)

[K] Y. Kuramoto, Chemical oscillations, waves, and turbulence, Springer Series in Synergetics, vol. 19, Springer-Verlag, Berlin, 1984. MR762432 (87e:92054)

[KT] Y. Kuramoto and T. Tsuzuki. On the formation of dissipative structures in reactiondiffusion systems. Progr. Theoret. Phys., 1975. 54:3.

[KSF] E. A. Kuznetsov, M. D. Spector, and G. E. Fal'kovich, On the stability of nonlinear waves in integrable models, Phys. D 10 (1984), no. 3, 379-386, DOI 10.1016/01672789(84)90186-6. MR763479 (86d:35131)

[NTY] Takaaki Nishida, Yoshiaki Teramoto, and Hideaki Yoshihara, Hopf bifurcation in viscous incompressible flow down an inclined plane, J. Math. Fluid Mech. 7 (2005), no. 1, 29-71, DOI 10.1007/s00021-004-0104-z. MR2127741 (2005m:76049)

[MZ] Corrado Mascia and Kevin Zumbrun, Pointwise Green function bounds for shock profiles of systems with real viscosity, Arch. Ration. Mech. Anal. 169 (2003), no. 3, 177263, DOI 10.1007/s00205-003-0258-5. MR2004135 (2004h:35137)

[M] Daniel Michelson, Stability of the Bunsen flame profiles in the Kuramoto-Sivashinsky equation, SIAM J. Math. Anal. 27 (1996), no. 3, 765-781, DOI 10.1137/0527041. MR $1382832(97 \mathrm{~b}: 80009)$

[NR1] P. Noble and L. M. Rodrigues, Whitham's equations for modulated roll-waves in shallow flows, ArXiv e-prints, arXiv:1011.2296v1, 2010.

[NR2] P. Noble and L.-M. Rodrigues, Whitham's Modulation Equations and Stability of Periodic Wave Solutions of the Generalized Kuramoto-Sivashinsky Equations, Indiana Univ. Math. J. 62 (2013), no. 3, 753-783. MR3164843

[OZ] M. Oh and K. Zumbrun, Stability of periodic solutions of conservation laws with viscosity: analysis of the Evans function, Arch. Ration. Mech. Anal. 166 (2003), no. 2, 99-166, DOI 10.1007/s00205-002-0216-7. MR:1957127(2004c:35270a)

[PSU] Robert L. Pego, Guido Schneider, and Hannes Uecker, Long-time persistence of Korteweg-de Vries solitons as transient dynamics in a model of inclined film flow, Proc. Roy. Soc. Edinburgh Sect. A 137 (2007), no. 1, 133-146, DOI 10.1017/S0308210505001113. MR2359776 (2009b:35365)

[PZ] Ramon Plaza and Kevin Zumbrun, An Evans function approach to spectral stability of small-amplitude shock profiles, Discrete Contin. Dyn. Syst. 10 (2004), no. 4, 885-924, DOI 10.3934/dcds.2004.10.885. MR2073940 (2005d:35169)

[Se] Denis Serre, Spectral stability of periodic solutions of viscous conservation laws: large wavelength analysis, Comm. Partial Differential Equations 30 (2005), no. 1-3, 259-282, DOI 10.1081/PDE-200044492. MR2131054(2006f:35178)

[SK] Yasushi Shizuta and Shuichi Kawashima, Systems of equations of hyperbolic-parabolic type with applications to the discrete Boltzmann equation, Hokkaido Math. J. 14 (1985), no. 2, 249-275. MR798756 (86k:35107)

[S1] G. I. Sivashinsky, Nonlinear analysis of hydrodynamic instability in laminar flames. I. Derivation of basic equations, Acta Astronaut. 4 (1977), no. 11-12, 1177-1206. MR0502829 (58 \#19741)

[S2] G. I. Sivashinsky, Instabilities, Pattern Formation, and Turbulence in Flames, Annu. Rev. Fluid Mech. 15 (1983), 179-199.

[SM] G. I. Sivashinsky and D. M. Michelson, On irregular wavy flow of a liquid down an vertical plane, Progr. Theoret. Phys. 63 (6) (1980), 2112-2114.

[Sp] M. D. Spektor, Stability of conoidal [cnoidal] waves in media with positive and negative dispersion (Russian), Zh. Èksper. Teoret. Fiz. 94 (1988), no. 1, 186-202; English transl., Soviet Phys. JETP 67 (1988), no. 1, 104-112. MR960877 (90e:35156)

[W] G. B. Whitham, Linear and nonlinear waves, Wiley-Interscience [John Wiley \& Sons], New York, 1974. Pure and Applied Mathematics. MR.0483954 (58 \#3905)

[Wi] Htay Aung Win, Model equation of surface waves of viscous fluid down an inclined plane, J. Math. Kyoto Univ. 33 (1993), no. 3, 803-824. MR.1239094 (95e:35171) 
[YY] Jun Yu and Yi Yang, Evolution of small periodic disturbances into roll waves in channel flow with internal dissipation, Stud. Appl. Math. 111 (2003), no. 1, 1-27, DOI 10.1111/1467-9590.t01-2-00225. MR1985993 (2004d:76039)

[Yo] W.-A. Yong, Basic properties of hyperbolic relaxation systems, Birkhauser's Series: Progress in Nonlinear Differential Equations and their Applications (2001), 207 pp.

[Ze] Yanni Zeng, Gas dynamics in thermal nonequilibrium and general hyperbolic systems with relaxation, Arch. Ration. Mech. Anal. 150 (1999), no. 3, 225-279, DOI 10.1007/s002050050188. MR1738119 (2000k:35173)

[Z] Kevin Zumbrun, Stability of detonation profiles in the ZND limit, Arch. Ration. Mech. Anal. 200 (2011), no. 1, 141-182, DOI 10.1007/s00205-010-0342-6. MR2781588 (2011m:35154)

Department of Mathematics, University of Kansas, 405 Snow Hall, 1460 Jayhawk Boulevard, LAWrence, KANSAS 66046

E-mail address: matjohn@ku.edu

Institut Camille Jordan, UMR CNRS 5208, Université de Lyon, Université Lyon I, 43 BD du 11 novembre 1918, F - 69622 Villeurbanne Cedex, France

E-mail address: noble@math.univ-lyon1.fr

Current address: Institut de Mathématiques de Toulouse, UMR CNRS 5219, INSA de Toulouse 135, avenue de Rangueil, 31077 Toulouse Cedex 4, France

E-mail address: Pascal.Noble@math.univ-toulouse.fr

Institut Camille Jordan, UMR CNRS 5208, Université de Lyon, Université Lyon 1, 43 BD Du 11 novembre 1918, F - 69622 Villeurbanne Cedex, France

E-mail address: rodrigues@math.univ-lyon1.fr

Department of Mathematics, Indiana University, 831 E. 3Rd Street, Bloomington, INDIANA 47405

E-mail address: kzumbrun@indiana.edu 